\title{
Traip Mitotic Function Controls Brain Size
}

\author{
Ryan S. O'Neill and Nasser M. Rusan
}

Cell and Developmental Biology Center, National Heart Lung and Blood Institute, National

Institutes of Health, Bethesda, MD 20892, USA

\section{Correspondence}

oneillrs@nih.gov (R.S.O.),nasser@nih.gov (N.M.R.) 


\section{Abstract}

Microcephaly is a developmental failure to achieve proper brain size and neuron number. Mutations in diverse genes are linked to microcephaly, including several with DNA damage repair (DDR) functions; however, it is not well understood how these DDR gene mutations limit brain size. One such gene is TRAIP, which has multiple known functions in DDR. We characterized the Drosophila ortholog Traip, finding that loss of Traip causes a brain-specific defect in the Mushroom Body (MB). Traip mutant (traip-) MBs had reduced size and fewer neurons, but no neurodegeneration, consistent with human primary microcephaly disorders. Reduced neuron numbers in traip were explained by premature caspase-dependent cell death of MB neuroblasts (MB-NBs). Many traip- MB-NBs had prominent chromosome bridges in anaphase, along with polyploidy, aneuploidy, or micronuclei. We found no evidence for an interphase DNA repair role for Traip in MB-NBs; instead, proper MB development requires Traip function during mitosis, where Traip localizes to centrosomes and mitotic spindles. Our results suggest that proper brain size is ensured by the recently described role for TRAIP in unloading stalled replication forks in mitosis, which suppresses DNA bridges and neural stem cell death to promote proper neuron number. Further, the mitotic nature of traip- MB-NB defects and Traip localization suggest a closer etiological link between DDR microcephaly genes like Traip and the centrosome/spindle-related genes more commonly associated with microcephaly. 


\section{Introduction}

Microcephaly is a developmental growth disorder characterized by reduced cerebral cortex size and neuron number. Mutations in about 40 genes are linked to heritable forms of microcephaly, ranging from MicroCephaly, Primary, Hereditary (MCPH; Jayaraman et al., 2018) where only brain size is reduced, to more severe forms like Seckel syndrome (SCKL) where both brain and body are reduced (Khetarpal et al., 2016). Most microcephaly disorders are linked to mutations in genes from one of two functional classes: centrosome and mitotic spindle functions, or DNA damage repair (DDR). Mutations in centrosome/mitotic spindle genes are thought to disrupt mitotic spindle structure, positioning, and orientation, leading to defects in chromosome segregation or cell cycle progression and ultimately neural progenitor cell (NPC) loss through premature differentiation or cell death (reviewed in Nano and Basto, 2017). Mutations in DDR genes are thought to increase DNA damage, activate checkpoint signaling and lead to increased genome instability, cell cycle lengthening, and the ultimate loss of NPCS (reviewed in Bianchi et al., 2018). However, in many cases microcephaly genes have not been directly studied in the context of brain development and the cellular etiology of microcephaly is inferred from studies in non-neuronal contexts. Thus, we aimed to study the microcephaly gene TRAIP in an animal to reveal the etiological mechanisms of disease.

TRAIP encodes a RING domain E3 ubiquitin ligase that localizes to the nucleus and functions in DDR. In humans, hypomorphic mutations in TRAIP cause SCKL with severe microcephaly and reduced body size (Harley et al., 2016). Cultured fibroblasts from TRAIP mutant patients have an elongated cell cycle and impaired DNA repair, suggesting that mutations in TRAIP cause microcephaly via reduced cell proliferation, premature differentiation, and increased apoptosis of NPCs (Harley et al., 2016). A mouse TRAIP null mutant is early embryonic lethal (Park et al., 2007) and Drosophila mutants for the TRAIP ortholog nopo (herein called Traip) are maternal effect lethal (homozygous null mothers lay eggs that arrest in the first few embryonic cell cycles; Merkle et al., 2009), whereas null mutant C. elegans are viable (Sonneville et al., 2019). No studies of TRAIP function in developing brains have been published. 
TRAIP has several functions related to DNA repair (reviewed in Wu et al., 2020). TRAIP localizes to DNA double-strand breaks, where it recruits other factors and promotes $\mathrm{H} 2 \mathrm{~B}$ monoubiquitylation to stimulate repair (Han et al., 2019; Soo Lee et al., 2016). TRAIP also localizes to stressed replication forks (Feng et al., 2016; Hoffmann et al., 2016) where it removes DNA-protein crosslinks via ubiquitylation (Larsen et al., 2019) and regulates the choice between NEIL3 or FANC/BRCA-mediated repair pathways (Wu et al., 2019). In addition to these interphase DDR functions, TRAIP also promotes proper sister chromatid separation during mitosis. A cell entering mitosis may have under-replicated sister chromatids (URSCs) which contain loci that failed to complete replication during interphase, and these URSCs must be resolved to prevent DNA bridges during anaphase. TRAIP initiates the resolution of URSCs by ubiquitylating the MCM7 subunit of CMG helicase, thus triggering replication machinery unloading from stalled forks and allowing sister chromatid resolution via mitotic DNA repair synthesis (Deng et al., 2019; Priego Moreno et al., 2019; Sonneville et al., 2019; Villa et al., 2021).

However, most previous studies on TRAIP function were done in cell culture, Xenopus egg extracts, and the early embryos of Drosophila and C. elegans, and thus it is unclear which TRAIP functions are critical for neurogenesis. In this study we take advantage of the well characterized brain structure and genetic tractability of Drosophila to establish a model of Traip mutant microcephaly. In this context, Traip functions in NSCs during mitosis to suppress DNA bridges and prevent premature caspase-dependent cell death, pointing to a role in URSC resolution as critical for proper brain size.

\section{Results}

\section{Traip is Required for Proper Mushroom Body Development}

We generated a new full coding sequence deletion of Traip using CRISPR (traip ${ }^{\Delta}$ ). We use traip to refer to traip ${ }^{\Delta}$ in combination with either the previously described null allele traip ${ }^{\text {Exc142 }}$ 
(Merkle et al., 2009) or the deficiency Df(2R)Exel7153 (Parks et al., 2004), as both allelic combinations showed identical brain phenotypes.

To test whether Traip controls brain size, we used $\mu$-computed tomography ( $\mu$-CT; Schoborg et al., 2019) to compare control and traip adult brains using normalized volumetric analysis (Figure 1A). While optic lobes were not different (Figure 1B), traip- central brain volume was slightly reduced, and this reduction was rescued by a ubiquitously expressed GFP-tagged Traip transgene (ubi-GFP::Traip; Figure 1C). These initial results suggested that Traip is required for fully proper brain development.

To determine if any specific central brain sub-region was disrupted, we examined neuropil ( $\mathrm{N}$ cadherin; CadN) and axon tracts (Neuroglian; Nrg) in traip- brains. All brain regions appeared qualitatively similar (Figure S1A), except for a 100\% penetrant size and structure defect in traip Mushroom Bodies (MBs), a pair of neuropils that mediate higher order functions in the insect brain (Figure 1D; Heisenberg, 2003; Modi et al., 2020). In Drosophila, each MB arises from four neuroblasts (MB-NBs), which divide continuously from embryonic development through late pupal stages to produce roughly 2500 Kenyon cells (KCs) per hemisphere (Figure 1E). These KCs are positioned on the posterior dorsal side of the brain and project axons in a stereotypic, bifurcated pattern to form the $\psi$ (first born), $\alpha^{\prime} / \beta^{\prime}$, and $\alpha / \beta$ (last born) lobes of the MB (Figure 1D).

The MBs of traip adult brains were misshapen and reduced in size (Figure 1G), with additional axon-related defects: $50 \%$ with axon tracts exiting the lobes ( $0 \%$ in controls), $93 \%$ with lobes fused at the midline ( $14 \%$ in controls), and $29 \%$ with missing $\alpha$ or $\beta$ lobes ( $0 \%$ in controls). traip $\alpha / \beta$ lobe volume, identified by Fasll staining, was reduced by $70 \%$ (Figure $1 \mathrm{H}$ and $1 \mathrm{I}$ ), and traip full MB volume, identified by mCD8::GFP expression driven in KCs via OK107-GAL4, was reduced by $37 \%$ (Figure S1B and S1C). The more severe size reduction of the last born, Fasll-positive $\alpha / \beta$ lobes indicates that the traip- MB defect progressively accumulates through development. Cross-sectional area was also reduced in Fasll-positive $\alpha$ lobes by $84 \%$ (Figure 1J) and in OK107GAL4 > mCD8::GFP $\alpha^{\prime} / \alpha$ lobes by 54\% (Figure S1D). 
MB size reduction was consistent across all Traip null mutant combinations (Figure S1E), whereas hypomorphic mutants (Merkle et al., 2009) had wild-type MB size (Figure S1E and S1F). MB size was rescued by ubiquitously expressing GFP::Traip (Figure S1G and S1H), and importantly, by specifically expressing GFP::Traip in the MB-NBs and KCs via OK107-GAL4 (Figure $1 \mathrm{I}-1 \mathrm{~K}$ ). Thus, MB size reduction is linked to cell-autonomous loss of Traip function in the MB-NBs and/or KCs.

Reduced traip- MB lobe size suggests fewer axons and reduced KC number. We used machine learning to count KCs with OK107-GAL4 > NLS::mCherry (Figure 1L). Each traip- hemisphere contained an average of 916 KCs, a 60\% reduction compared to controls with $2260 \mathrm{KCs}$ (Figure 1M). Regression analyses showed a significant linear correlation between KC number and both MB volumes (Figure S2I) and cross sectional areas (Figure S2J). Having shown that $\alpha$ lobe crosssectional area measurement was a suitable proxy for KC number, we used this measurement for the remainder of this study.

\section{Traip is Required in Mushroom Body Neuroblasts During Development}

To determine whether the traip- MB size reduction was developmental (primary microcephalylike) or neurodegenerative (secondary microcephaly-like; Passemard et al., 2013), we investigated pre-adult stages (Figure 2A). MB size was not significantly reduced in traip- larvae, but was markedly reduced through pupal stages (Figure 2B). Thus, traip- is primary microcephaly-like, as MB size reduction arises during development.

To investigate a possible role in neurodegeneration we compared the MBs of three day old and 50 day old adults (Figure 2C). MB size increased slightly with age in controls (Figure 2D), reflecting neuropil reorganization in response to life experience (Heisenberg et al., 1995). In contrast, traip- MBs neither grew nor shrunk with age (Figure 2D). Thus, traip ${ }^{-}$MB size reduction is not neurodegenerative and does not reflect secondary microcephaly.

We hypothesized that traip- reduced KC number and MB size could arise in two ways: 1) a defect in the highly mitotic MB-NBs resulting in fewer KCs born, and 2) a defect in the 
differentiated KCs resulting in cell death. We began by exploring which cell types express Traip, using CRISPR to knock-in mNeonGreen at the endogenous Traip locus (mNG::Traip), which resulted in a fly line with wild-type MB morphology (Figure S2A). mNG::Traip was expressed in all $3^{\text {rd }}$ instar larval central brain NBs (CB-NBs), which include the MB-NBs (Figure 3A). In addition, mNG::Traip expression persisted in the mitotic ganglion mother cells (GMCs) and their immediate daughter neurons (Figure 3B). However, mNG::Traip was absent from regions of mature neurons in larval brains (Figure 3A), and was not detectable in adult brain neurons (Figure S2B). These data suggest that Traip primarily functions in proliferating brain cells.

To determine which cell types require Traip function, we used cell type-specific expression of GFP::Traip in attempt to rescue traip- MB size. First, we used wor-GAL4 to drive UAS-GFP::Traip in NBs, which rescued MB size (Figure 3C and 3D). However, this did not fully exclude a role for Traip in neurons, as wor-GAL4 > GFP::Traip was also found in the GMCs and newly born neurons (Figure S2C). We next tried expressing GFP::Traip in post-mitotic neurons using elav-GAL4, but discovered strong expression in the NBs and GMCs (Figure S2D). Finally, we used nSyb-GAL4 to specifically drive GFP::Traip expression in mature neurons (Figure S2E), which resulted in failure to rescue MB size (Figure 3E and 3F). Thus, Traip function in neurons alone is insufficient to prevent microcephaly. We conclude that the primary function for Traip is in proliferating brain cells (NBs and GMCs), albeit we could not fully rule out the possibility that undetectable levels of Traip also function in post-mitotic neurons.

\section{Traip Mutant Mushroom Body Neuroblasts are Lost via Caspase-Dependent Cell Death}

We next hypothesized that traip- reduction in $\mathrm{KC}$ number and $\mathrm{MB}$ size is due to premature loss of MB-NBs. The KCs of each MB arise from four MB-NBs that are easily identifiable at 24 hours after pupal formation (APF), as nearly all other NBs are lost by this stage (Ito and Hotta, 1992; Truman and Bate, 1988). Controls maintained four MB-NBs throughout pupal development (Figure 4A and 4B). In contrast, traip- progressively lost their MB-NBs, with an average 2.2 MBNBs per hemisphere at 24 hours APF decreasing to 0.9 by 72 hours APF, and this loss was rescued by OK107-GAL4 > UAS-GFP::Traip (Figure 4A and 4B). 
To explore the role of caspase-dependent cell death in MB-NB loss we used $D f(3 L) H 99$, which deletes four pro-apoptotic genes (grim, rpr, hid, and skl; Abbott and Lengyel, 1991). MB-NB loss was suppressed in traip ${ }^{-}$with $D f(3 L) H 99$ compared to traip ${ }^{-}$alone at 24 hours APF (3.1 vs 2.2; Figure $4 A$ and $4 B$ ), although the effect is not observed in later stages. $D f(3 L) H 99$ also improved traip $^{-M B}$ size more than 2-fold (Figure 4D and 4E). Since $D f(3 L) H 99$ deletes other genes, we also used RNAi against the effector caspase Drice, which improved traip- MB size by 1.6-fold (Figure 4E and 4F). Together, these data support a model where Traip prevents premature caspase-dependent cell death of MB-NBs to ensure proper $\mathrm{KC}$ number and MB morphology.

\section{Traip Suppresses Chromosome Bridges During Anaphase}

After noticing abnormal nuclei while scoring traip- MB-NB number, we characterized nuclear phenotypes in 24 hours APF MB-NBs to gain insight into defects potentially upstream of MB-NB death. Control MB-NBs had nuclei with relatively smooth, spherical nuclear lamina morphology and no other defects (100\%; Figure 5A). In contrast, some traip- MB-NBs appeared apoptotic, with abnormally condensed DAPI staining (11\%; Figure 5B), while others frequently had nuclear defects including ruffled, convoluted nuclear lamina morphology (12\%; Figure 5C), micronuclei (11\%; Figure 5D), multiple nuclei (9\%; Figure 5E), or extremely large nuclei suggestive of polyploidy (9\%; Figure 5F). These traip- phenotypes were rescued by OK107-GAL4 > UASGFP::Traip (Figure 5G).

As these nuclear defects could arise during mitosis, we used high resolution live fluorescence microscopy to analyze mitotic dynamics of $3^{\text {rd }}$ instar larval CB-NBs. Unlike control NBs (Figure $5 \mathrm{H}$ and Movie 1), $26 \%$ of traip NBs had prominent chromosome bridges, where sister chromatids appeared attached and did not effectively segregate, but rather became stretched across the midzone through anaphase before eventually separating (Figure 5I, Movie 2). Further, traip- larval brains often had cells with abnormally large nuclei (Figure S3A), reminiscent of the suspected polyploid cells seen in traip- pupal brains (Figure 5E). We reasoned that traip- mitotic DNA bridges could block cytokinesis, resulting in multiple nuclei or polyploidy, or else lead to chromosome breakage, resulting in micronuclei. 
We also found evidence suggesting persistent aneuploidy in traip- MB-NBs. Control pupae expressing both mCD8::GFP and NLS::mCherry via OK107-GAL4 had similar fluorescence levels across the field of mature KCs (Figure 5J). In contrast, traip- pupae often contained one or more clusters of KCs that had either double the signal or no signal for the GFP or mCherry marker (Figure 5K). These aberrant KC clusters were reminiscent of clonal cell clusters, suggesting that their MB-NB progenitors became aneuploid, either losing or gaining a copy of one transgene, and then continued generating daughter cells. These clones were never observed in controls, whereas traip brains had an average of 1.3 clones per hemisphere at 24 hours and 1.7 clones per hemisphere at 72 hours APF (Figure $5 \mathrm{~L}$ ). The average number of KCs per clone in traip brains increased from 15 at 24 hours to 37 at 72 hours APF (Figure S3B), with some clones containing hundreds of KCs. OK107-GAL4 > UAS-GFP::Traip suppressed clone formation (Figure $5 \mathrm{~L})$. These data are consistent with traip- mitotic DNA bridges leading to aneuploid MB-NBs which often continue producing daughters before eventually being lost.

We next used live imaging to explore whether traip- NBs were defective in mitotic timing, but found no difference in the duration from prophase onset to completion of furrow constriction for CB-NBs (Figure S3C). Additionally, the presence or absence of DNA bridges in traip- NBs did not affect mitotic timing from anaphase onset to furrow constriction (Figure S3D), although we could not rule out a possible delay or failure in abscission. In fixed larval brains, the mitotic index of control and traip- CB-NBs were similar (Figure S3E). In 24 hours APF brains, the mitotic index of traip MB-NBs was lower than controls (20\% traip- vs 35\% controls); however, when MB-NBs with nuclear defects (Figure 5B-F) were excluded from this analysis, the mitotic index of phenotypically normal traip- MB-NBs was not different from controls (32\% traip- vs 35\% controls; Figure S3F). Thus, the reduced mitotic index of traip- MB-NBs could be explained by an inability of polyploid, multinucleate, and apoptotic cells to enter mitosis rather than a function for Traip in directly controlling mitotic or cell cycle timing.

\section{Traip Functions to Resolve Under-Replicated Sister Chromatids}


TRAIP has a known role in resolving under-replicated sister chromatids (URSCs) at mitotic onset (Deng et al., 2019; Priego Moreno et al., 2019; Sonneville et al., 2019), and URSCs are predicted to form a special class of mitotic DNA bridges called ultrafine DNA bridges (UFBs; Liu et al., 2014). To test for UFBs we generated transgenes encoding fluorescently tagged FancD2; human FANCD2 localizes as puncta at the points where UFBs connect with sister chromatids (Naim and Rosselli, 2009), and although FancD2 localization has not been well-characterized in Drosophila, there is precedence for invisible DNA tethers in Drosophila cells (Royou et al., 2010). We found that mNG::FancD2 puncta localized to mitotic DNA bridges in many traip- NBs (7/12; Figure 6B), in addition to weakly localizing to mitotic chromosomes in both controls and traip- NBs (Figure $6 \mathrm{~A}, 6 \mathrm{~B})$. The observed FancD2 on chromosome bridges suggests the presence of UFBs and is consistent with a role for Traip in resolving URSCs at mitotic onset.

To better understand the mechanism of URSC and mitotic DNA bridge formation, we used drug treatments to induce replication stress via various mechanisms in control and traip- larvae and assayed their survival to adult (Figure 6C). A major source of URSCs is late replicating regions, where replication forks fail to converge before mitotic entry (Liu et al., 2014); hydroxyurea increases the number of late replicating regions by depleting the nucleotide pool and inhibiting replication (Bianchi et al., 1986). Surprisingly, traip- mutants were not sensitive to hydroxyurea treatment (Figure S4A), suggesting that late replicating region-induced URSCs are not the major target for Traip.

Another source of URSCs is inter-strand crosslinks, which block replication machinery and cause forks to stall; both Cisplatin and Mitomycin C are inter-strand crosslinking agents (Deans and West, 2011). Whereas controls tolerated high doses of Cisplatin and Mitomycin C, traip- animals only tolerated low doses, with higher doses causing lethality (Figure 6D), likely due to defects in imaginal disc proliferation (Figure S4B). The MBs of traip ${ }^{-}$adults raised on medium doses of Cisplatin or Mitomycin C were not affected (Figure S4C, S4D); however, this may be due to a failure of these drugs to cross the blood brain barrier, as is the case in mammals (Gregg et al., 1992; Reddy and Randerath, 1987). Nonetheless, these experiments indicate that Traip functions to resolve DNA crosslink-induced damage. 
TRAIP triggers replication machinery unloading via ubiquitylation of MCM7 (Wu et al., 2019), and therefore requires an intact RING domain for E2 conjugating enzyme binding. Thus, we tested whether the RING domain is required for Traip function in MB development. GFP::Traip ${ }^{L 12 E, G 14 D}$, which is a control mutant predicted to have a functional RING domain, fully rescued traip ${ }^{-} \mathrm{MB}$ size (Figure 6E and 6F). In contrast, GFP::Traip ${ }^{18 D, A 10 E}$, which is predicted to have a non-functional RING-E2 binding interface, failed to rescue traip- MB size (Figure 6F and 6G). Interestingly, GFP::Traip ${ }^{\triangle R I N G}$ enhanced the traip- $M B$ size defect (Figure $6 \mathrm{H}$ ), indicating a dominant negative effect of deleting the RING domain. These results show that Traip function in MB-NBs is RING-dependent, consistent with an E3 ligase function and role in replication machinery unloading.

\section{Proper MB Development requires mitotic but not interphase Traip function}

We discovered a novel mitotic localization for Traip while characterizing mNG::Traip expression. In interphase, mNG::Traip localized to the nucleus, as previously described (Feng et al., 2016; Harley et al., 2016; Soo Lee et al., 2016). However, in mitosis mNG::Traip localized to mitotic spindles and concentrates at the centrosomes (Figure 7A and Movie 3). High resolution imaging revealed that $\mathrm{mNG}::$ Traip forms small puncta that travel poleward along the spindles (Figure 7B and Movie 4) and also coalesce at the cytokinetic furrow and midbody (Figure 7C) in late mitosis.

To characterize regions within Traip responsible for its mitotic localizations, we expressed GFP::Traip variants in traip CB-NBs via wor-GAL4, including Full Length (FL), RING mutant (I8D, A10E), and several truncations and internal deletions based on the major features of Traip (Figure S5A). GFP::Traip ${ }^{\mathrm{FL}}$ recapitulated the interphase and mitotic localizations of the mNG::Traip CRISPR transgenic (Figure 7D and S5B). GFP::Traip ${ }^{18 D, A 10 E}$ localized to the proper sites, but formed some aggregates (Figure S5C). Neither the RING domain nor the first coiled coil were sufficient to mediate any localization (Figure S5D). Both the second coiled coil and the C-terminal region were sufficient to mediate centrosome localization (Figure S5E-S5H). Finally, the C-terminal region was both necessary and sufficient for localization to the furrow during 
mitosis and the nucleus/nucleolus during interphase (Figure S5G and S5H). We also tested whether these protein regions are required for Traip MB function, but none were sufficient to rescue traip- MB size (Figure S6A) indicating that Traip function requires multiple domains. Human GFP::TRAIP also failed to rescue traip- MB size (Figure S6A).

Reasoning that Traip could control MB development through either an interphase or a mitotic function, we first tested whether Traip has any major DDR functions in interphase. Using $\mathrm{\gamma H}^{2 \mathrm{Av}}$ staining to identify double stranded DNA breaks in 24 hours APF MB-NBs, we found that control (Figure S7A) and most traip- (Figure S7B) MB-NBs had few $\mathrm{\gamma H} 2 \mathrm{Av}$ puncta. A small subset of traip ${ }^{-}$MB-NBs had extremely elevated $\mathrm{pH} 2 \mathrm{Av}$ puncta (Figure S7C); however, these cells appeared to be apoptotic, and thus likely reflected an indirect effect of traip- (caspasedependent cell death) rather than a direct effect of impaired interphase DNA repair. Excluding these outliers, $\mathrm{yH}_{2} \mathrm{Av}$ puncta numbers were not different between controls and traip- (Figure S7D). We also hypothesized that if Traip functions in interphase DNA repair, then activation of DNA damage signaling might potentiate cell death in traip- MB-NBs. However, knocking down mei-41, chk1, or $p 53$ via RNAi did not significantly improve traip- MB size (Figure S7E). These data suggest that interphase DNA repair is not a major function for Traip in MB-NBs under normal conditions.

We next tested whether Traip primarily controls MB development through a mitotic function. GFP::Traip ${ }^{\triangle N L S}$, which contains a deletion of the nuclear localization signal (NLS), localized similarly to wild type GFP::Traip during mitosis but was cytoplasmic in interphase (Figure 7F). Nonetheless, GFP::Traip ${ }^{\triangle N L S}$ fully rescued traip- $\mathrm{MB}$ size (Figure $7 \mathrm{G}$ and $7 \mathrm{H}$ ), indicating that a mitotic Traip function is critical for MB-NBs. To test whether proper mitotic localization is important for Traip function, we introduced a Tom20 tag to ectopically force Traip to the mitochondria. Tom20::TagRFP::Traip localized cytoplasmically in interphase, and was absent from the spindle region in mitosis (Figure 7l). Mitochondria-localized Tom20::TagRFP::Traip provided an intermediate rescue of traip $\mathrm{MB}$ size (Figure $7 \mathrm{H}$ and $7 \mathrm{~J}$ ). Thus, proper mitotic localization of Traip to the spindle, centrosome, and/or midbody is important for full Traip function. 


\section{Discussion}

Our study in Drosophila shows that traip shares several characteristics with human microcephaly mutants. First, the traip- phenotype is highly brain-specific, with body defects being extremely rare. Second, the traip ${ }^{-}$MB phenotype is developmental rather than neurodegenerative in nature, reflecting a primary rather than secondary microcephaly-like disorder. Finally, as with many human microcephaly genes, Traip functions to promote neural progenitor cell (NPC) proliferation and survival. Thus, traip- represents a powerful new disease model for understanding the etiological mechanisms underlying microcephaly.

Despite their ubiquitous expression, mutations in microcephaly genes primarily affect the cerebral cortex in humans. Similarly, both Traip and the DDR microcephaly gene MCPH1 (Rickmyre et al., 2007) are ubiquitously expressed in Drosophila, yet the MB is the only adult structure affected in their mutants. While most NBs have a limited window of proliferation, MB-NBs divide continuously from embryogenesis into late pupal stages (Ito and Hotta, 1992; Truman and Bate, 1988), potentially allowing more accumulation of rare or small effects over many cell cycles. Interestingly, our drug experiments indicate that traip- mitotic DNA bridges may be the result of inter-strand crosslinks rather than late replicating regions; perhaps the highly proliferative MB-NBs generate increased reactive oxygen species and aldehydes, leading to increased inter-strand crosslinks. Additionally, while many tissues can make up for lost cells via compensatory proliferation (Haynie and Bryant, 1977; Pfau et al., 2016), no such process appears to exist for replacing lost NPCs. Thus, we speculate that the MBs are especially sensitive to mutations in microcephaly genes as a consequence of rapid, prolonged proliferation and inability to compensate for lost progenitor cells, and that a similar explanation may account for the sensitivity to microcephaly gene mutation in the human cortex.

Our work provides the first link between a known function of Traip and proper brain development. We found that interphase nuclear localization is not required for Traip function, interphase double stranded DNA breaks are not increased in traip ${ }^{-}$MB-NBs, and DNA damage signaling does not appear to mediate traip ${ }^{-}$MB-NB death, and thus conclude that Traip interphase functions are dispensable for MB-NB survival under normal conditions. Instead, we 
discovered the presence of mitotic DNA bridges, sensitivity to inter-strand crosslinking agents, RING domain-dependence, and requirement for mitotic localization only, and therefore conclude that the primary function for Traip in MB-NBs is to ubiquitylate and remove stalled replication machinery during mitosis (Figure 8A; Deng et al., 2019; Priego Moreno et al., 2019; Sonneville et al., 2019). Thus, we surmise that traip- MB-NBs have stalled replication machinery which remains loaded throughout mitosis, preventing mitotic DNA synthesis repair and proper sister chromatid segregation in mitosis (Figure 8B). As anaphase proceeds, attached sister chromatids are pulled to opposite poles and they form UFBs as the under-replicated DNA is stretched out between them. These bridges could be physically broken, leading to chromosome fragmentation, generating aneuploidy or micronuclei and causing nuclear deformations in daughter cells (Gisselsson et al., 2001; Heddle and Carrano, 1977). Alternatively, persistence of DNA bridges at the cytokinetic furrow could induce mitotic exit and furrow regression (Pampalona et al., 2012; Shi and King, 2005), leading to multiple nuclei or polyploidy which likely prevent further proliferation.

We found that traip- MB-NBs are prematurely lost via caspase-dependent cell death and thus fail to generate proper KC numbers. DNA bridge-induced defects likely feed into apoptosis, but further work is required to dissect the pathways connecting them. In Drosophila, polyploid NBs can accumulate significant DNA damage as they enter mitosis (Nano et al., 2019), and chromosome breakage during mitosis in traip could induce death through DNA damage signaling. Drosophila embryos laid by traip mothers are lethal, with extensive chromosome bridging and Chk2-dependent cell death, suggesting DNA damage accumulation leads to apoptosis in the rapidly dividing cells of the early embryo (Merkle et al., 2009). However, our RNAi experiments in the MB indicate that DNA damage signaling is not a major contributor to MB-NB apoptosis (Figure S7E). In mammalian NPCs, polypoidy and binucleation can cause G1 arrest and apoptosis (Aylon and Oren, 2011; Storchova and Kuffer, 2008). In Drosophila, neurons can become polyploid in response to DNA damage (Nandakumar et al., 2020), and NBs can become massively polyploid in some mutants (Poulton et al., 2017; Swider et al., 2019), suggesting that, even though polyploidy may be better tolerated in flies, polyploid NBs are unlikely to successfully complete additional mitoses. We infer the existence of traip aneuploid 
MB-NBs which produce a wide range of daughter KC numbers, suggesting that traip generates some aneuploidies that are well-tolerated and others that are highly lethal to the cell. This parallels the situation in mammals, where aneuploid NPCs and neurons are common (Rehen et al., 2001) but also sensitive to G1 arrest, cell cycle exit, and apoptosis (Peterson et al., 2012; Storchova and Kuffer, 2008). Thus, both polyploidy and aneuploidy could stop further proliferation in traip- MB-NBs by preventing proper mitosis or inducing G1 arrest and cell cycle exit, eventually triggering apoptosis.

In our study we identified a centrosome, spindle, and cytokinetic furrow localization for Traip which is important for function. One possibility is that the dynamic movement of Traip on the mitotic spindle and cytokinetic furrow promotes encounters with unresolved DNA bridges. We never observed GFP::Traip on bridges; however, it is thought that a single TRAIP protein is sufficient to unload each replisome (Wu et al., 2019), so fluorescence detection may be unlikely. Interestingly, centrosome localization is a common aspect of microcephaly-linked proteins, including MCPH1 which also functions in DDR (Jeffers et al., 2008; Rai et al., 2008). Similar to Traip, MCPH1 has mitotic functions required for proper chromosome segregation, and mutations in MCPH1 lead to lagging chromosomes, DNA bridges, and micronuclei (Arroyo et al., 2017; Rickmyre et al., 2007). Mutations in microcephaly genes with centrosomeassociated functions, such as CEP135 and CDK5RAP2, cause dysregulation of centrosome numbers which also lead to chromosome segregation errors and aneuploidy (Barrera et al., 2010; Hussain et al., 2012; Shi et al., 2019). Thus, mitotic roles, ensuring proper chromosome segregation, and suppressing aneuploidy are common features of microcephaly-linked proteins. Future work seeking to better understand these shared defects may reveal a deeper etiological connection across microcephaly disorders. 


\section{Materials and Methods}

\begin{tabular}{|c|c|c|}
\hline REAGENT or RESOURCE & SOURCE & IDENTIFIER \\
\hline \multicolumn{3}{|l|}{ Antibodies } \\
\hline mouse anti-Fasll & $\begin{array}{l}\text { Developmental Studies } \\
\text { Hybridoma Bank }\end{array}$ & 1D4 \\
\hline rat anti- CadN & $\begin{array}{l}\text { Developmental Studies } \\
\text { Hybridoma Bank }\end{array}$ & DN-Ex \#8 \\
\hline mouse anti-Nrg & $\begin{array}{l}\text { Developmental Studies } \\
\text { Hybridoma Bank }\end{array}$ & BP104 \\
\hline mouse anti-Elav & $\begin{array}{l}\text { Developmental Studies } \\
\text { Hybridoma Bank }\end{array}$ & 7E8A 10 \\
\hline mouse anti-Lamin & $\begin{array}{l}\text { Developmental Studies } \\
\text { Hybridoma Bank }\end{array}$ & ADL84.12 \\
\hline mouse anti- $\gamma \mathrm{H} 2 \mathrm{Av}$ & $\begin{array}{l}\text { Developmental Studies } \\
\text { Hybridoma Bank }\end{array}$ & UNC93-5.2.1 \\
\hline mouse anti-Tubulin & $\begin{array}{l}\text { Developmental Studies } \\
\text { Hybridoma Bank }\end{array}$ & E7 \\
\hline mouse anti-GFP & ThermoFisher & A11122 \\
\hline mouse anti-Phospho-tyrosine & Millipore & $4 G 10$ \\
\hline $\begin{array}{l}\text { rabbit anti-Phospho-histone } \\
\text { H3 }\end{array}$ & Millipore & H3S10P \\
\hline guinea pig anti-Dpn & Skeath lab & $\mathrm{N} / \mathrm{A}$ \\
\hline $\begin{array}{l}\text { Alexa Fluor 488/568/647 } \\
\text { conjugated secondary } \\
\text { antibodies }\end{array}$ & Thermo Fisher Scientific & $\begin{array}{l}\text { Variable host and target } \\
\text { species }\end{array}$ \\
\hline \multicolumn{3}{|l|}{$\begin{array}{l}\text { Chemicals, Peptides, and } \\
\text { Recombinant Proteins }\end{array}$} \\
\hline Alexa Fluor 568 Phalloidin & Molecular Probes/Fisher & Cat \# A12380 \\
\hline
\end{tabular}




\begin{tabular}{|c|c|c|}
\hline & Scientific & \\
\hline Phalloidin-Atto $647 \mathrm{~N}$ & Sigma Aldrich & Cat \# 65906 \\
\hline Cisplatin & Sigma Aldrich/Calbiochem & Cat \# 232120 \\
\hline Mitomycin C & Sigma Aldrich/Calbiochem & Cat \# 475820 \\
\hline Hydroxyurea & Sigma Aldrich/Calbiochem & Cat \# 400046 \\
\hline \multicolumn{3}{|l|}{ Drosophila Strains } \\
\hline$y w$ & Peifer Lab (UNC-Chapel Hill) & $\mathrm{N} / \mathrm{A}$ \\
\hline nopo ${ }^{\text {Exc142 }}$ & Merkle et al., 2009 & $\mathrm{~N} / \mathrm{A}$ \\
\hline nopo 21447 & $\begin{array}{l}\text { Bloomington Drosophila } \\
\text { Stock Center }\end{array}$ & BDSC: 57334 \\
\hline$D f(2 R)$ Exel7153 & $\begin{array}{l}\text { Bloomington Drosophila } \\
\text { Stock Center }\end{array}$ & BDSC: 7893 \\
\hline$D f(3 L) H 99$ & $\begin{array}{l}\text { Bloomington Drosophila } \\
\text { Stock Center }\end{array}$ & BDSC: 1576 \\
\hline mCherry RNAi & $\begin{array}{l}\text { Bloomington Drosophila } \\
\text { Stock Center }\end{array}$ & BDSC: 35787 \\
\hline Drice RNAi & $\begin{array}{l}\text { Bloomington Drosophila } \\
\text { Stock Center }\end{array}$ & BDSC: 32403 \\
\hline mei-41 RNAi & $\begin{array}{l}\text { Bloomington Drosophila } \\
\text { Stock Center }\end{array}$ & BDSC: 41934 \\
\hline chk1 RNAi & $\begin{array}{l}\text { Bloomington Drosophila } \\
\text { Stock Center }\end{array}$ & BDSC: 36685 \\
\hline p53 RNAi & $\begin{array}{l}\text { Bloomington Drosophila } \\
\text { Stock Center }\end{array}$ & BDSC: 41720 \\
\hline Tub-GAL4 & $\begin{array}{l}\text { Bloomington Drosophila } \\
\text { Stock Center }\end{array}$ & BDSC: 5138 \\
\hline OK107-GAL4 & $\begin{array}{l}\text { Bloomington Drosophila } \\
\text { Stock Center }\end{array}$ & BDSC: 854 \\
\hline wor-GAL4 & Bloomington Drosophila & BDSC: 56554 \\
\hline
\end{tabular}




\begin{tabular}{|c|c|c|}
\hline & Stock Center & \\
\hline elav-GAL4 & $\begin{array}{l}\text { Bloomington Drosophila } \\
\text { Stock Center }\end{array}$ & BDSC: 8760 \\
\hline$n S y b-G A L 4$ & $\begin{array}{l}\text { Bloomington Drosophila } \\
\text { Stock Center }\end{array}$ & BDSC: 51635 \\
\hline UAS-mCD8::GFP & $\begin{array}{l}\text { Bloomington Drosophila } \\
\text { Stock Center }\end{array}$ & BDSC: 32186 \\
\hline UAS-NLS::mCherry & Giniger Lab (NINDS-NIH) & N/A \\
\hline ubi-GFP::Tubulin & $\begin{array}{l}\text { Dr. Tomer Avidor-Reiss, } \\
\text { University of Toledo }\end{array}$ & $\mathrm{N} / \mathrm{A}$ \\
\hline ubi-moesin::GFP & Kiehart Lab (Duke) & N/A \\
\hline His2Av::mRFP & $\begin{array}{l}\text { Bloomington Drosophila } \\
\text { Stock Center }\end{array}$ & BDSC: 23650 \\
\hline UAS-mCherry::Tubulin & Rusan Lab & N/A \\
\hline $\begin{array}{l}M\{R F P[3 \times P 3 . P B] \\
G F P[E .3 \times P 3]=\text { vas-Cas9\}ZH-2A }\end{array}$ & $\begin{array}{l}\text { BestGene/Bloomington } \\
\text { Drosophila Stock Center }\end{array}$ & BDSC: 51323 \\
\hline \multicolumn{3}{|l|}{ Recombinant DNA } \\
\hline pPWG & $\begin{array}{l}\text { Drosophila Genomics } \\
\text { Resource Center }\end{array}$ & DGRC: 1078 \\
\hline pUC57 simple & GenScript & Cat \# SD1176 \\
\hline pUC6-chiRNA & Addgene & Plasmid \# 45946 \\
\hline pUC-attP-3xP3-TR & Rusan Lab & N/A \\
\hline pOT2-nopo & $\begin{array}{l}\text { BDGP Drosophila Gold } \\
\text { Collection }\end{array}$ & GH03577 \\
\hline FancD2 cDNA & Twist Bioscience & synthesized oligo \\
\hline hsTRAIP cDNA? & Dharmacon, Inc & MHS6278-202825899 \\
\hline pENTR & Thermo Fisher Scientific & Cat \# K2400-20 \\
\hline pPGW & $\begin{array}{l}\text { Drosophila Genomics } \\
\text { Resource Center }\end{array}$ & DGRC: 1077 \\
\hline
\end{tabular}




\begin{tabular}{|c|c|c|}
\hline pUGW & $\begin{array}{l}\text { Drosophila Genomics } \\
\text { Resource Center }\end{array}$ & DGRC: 1283 \\
\hline pUNW & Rusan Lab & $\mathrm{N} / \mathrm{A}$ \\
\hline pPT20TRW & Rusan Lab & $\mathrm{N} / \mathrm{A}$ \\
\hline \multicolumn{3}{|l|}{ Software and Algorithms } \\
\hline $\begin{array}{l}\text { MetaMorph for CSU-10 and } \\
\text { CSU-22 systems }\end{array}$ & Molecular Devices & \\
\hline Nikon Elements & Nikon & \\
\hline Zen Black & Zeiss & \\
\hline Skyscan & Bruker & \\
\hline $\begin{array}{l}\text { NRecon, Bruker MicroCT, } \\
\text { v1.7.0.4 }\end{array}$ & Bruker & \\
\hline FIJI/ImageJ & $\mathrm{NIH}$ & http://fiji.sc/ \\
\hline Dragonfly v3.6 & Object Research Systems & $\begin{array}{l}\text { http://www.theobjects.com/ } \\
\text { dragonfly/ }\end{array}$ \\
\hline Aivia & SVision & $\begin{array}{l}\text { https://www.aivia- } \\
\text { software.com/ }\end{array}$ \\
\hline Excel & Microsoft & $\begin{array}{l}\text { https://products.office.com/ } \\
\text { en-us/excel }\end{array}$ \\
\hline Flycrispr design tool & & $\begin{array}{l}\text { http://flycrispr.molbio.wisc.e } \\
\underline{\text { du/tools }}\end{array}$ \\
\hline cNLS Mapper & Kosugi et al., 2009 & $\begin{array}{l}\text { http://nls- } \\
\text { mapper.iab.keio.ac.jp/cgi- } \\
\text { bin/NLS Mapper form.cgi/ }\end{array}$ \\
\hline Prism 9 & GraphPad & $\begin{array}{l}\text { www.graphpad.com/scientifi } \\
\text { csoftware/prism/ }\end{array}$ \\
\hline Photoshop/Illustrator & Adobe & $\begin{array}{l}\text { www.adobe.com/uk/product } \\
\text { s/ }\end{array}$ \\
\hline
\end{tabular}




\section{D. melanogaster}

Experimental fly crosses were maintained on Bloomington Recipe Fly food from LabExpress (Ann Arbor, $\mathrm{MI}$ ) and kept at $25^{\circ} \mathrm{C}$. Crosses were either 8 virgin females per vial or 20 virgin females per bottle, with at least half as many males. yw was used as a control in all genotypes marked "+". All new transgenic animals were generated using standard embryo injection protocols by BestGene (Chino Hills, CA).

\section{Generation of Transgenic Drosophila}

For traip ${ }^{\Delta}$, homology arms were cloned into pUC-attP-3xP3-TR, such that the repair construct contained 1100 bp upstream of the Traip start codon and 1094 bp downstream of the Traip stop codon flanking a attP-3xP3-TR replacement. For $m N G:: T r a i p$, homology arms and mNeonGreen were cloned into pUC57, such that the repair construct contained $2226 \mathrm{bp}$ upstream of the Traip start codon, the mNeonGreen coding sequence, and $1093 \mathrm{bp}$ downstream of the Traip start codon. Guide RNAs were cloned into pU6-chiRNA. Note that PAM sequences in homology arms were mutated such that they were not predicted to be targets Cas9. Repair template and gRNA plasmid constructs were injected into $y^{1}$ $M\left\{R F P[3 \times P 3 . P B]\right.$ GFP[E.3xP3]=vas-Cas9\}ZH-2A $w^{1118} / F M 7 c$ using standard procedures. Putative CRISPR-positive alleles were crossed to CyO-Cre to eliminate 3xP3-TagRFP and were fully sequenced from outside the homology arms. traip ${ }^{\Delta}$ was further back-crossed to $y w$ for three generations and re-sequenced to eliminate a second site lethal mutation. Both CRISPR alleles were crossed to $D f(2 R)$ Exel7153 to test for maternal effect lethality; traip ${ }^{\Delta}$ was maternal effect lethal, and $m N G::$ Traip was fertile.

Traip $^{18 D, A 10 E}$ and Traip ${ }^{\angle 12 E, G 14 D}$ were rationally designed using amino acid alignment of Traip and TRAF6, which is a RING domain E3 ligase that has a known crystal structure with its E2 conjugating enzyme Ubc13 (Yin et al 2009). Traip ${ }^{\Delta N L S}$ was designed using cNLS Mapper, which predicts importin $\alpha$-dependent, CDK1-regulated NLS sequences (Kosugi et al 2009). Traip ${ }^{18 D, A 10 E}$, Traip $^{L 12 E, G 14 D}$ and Traip ${ }^{\triangle N L S}$ (deletion of positions 357-366 YSIFKKPRLL) were generated using 
mutagenic primers. Traip truncations were PCR amplified from cDNA. Traip, Traip variants, human TRAIP, and FancD2 cDNAs were cloned into pENTR using standard Gateway cloning methods. Gateway cloning was used to move constructs from pENTR into pPGW for UASGFP::Traip ${ }^{\text {variants }}$, pUGW for ubi-GFP::Traip, pPT20TRW for UAS-Tom20::TagRFP::Traip, and pUNW for ubi-mNG::FancD2.

\section{Drug Treatments}

Parent flies (traip ${ }^{E x c 142} / C y O$ females and either $y w$ or traip ${ }^{\Delta}$ males) were crossed for 2-3 days and then transferred to fly food made from $0.6 \mathrm{~g}$ of Carolina Formula 4-24 (Fisher Scientific) and $2 \mathrm{~mL}$ of $\mathrm{dd}_{2} \mathrm{O}$. Parents laid eggs for 24 hours, eggs were allowed to hatch for 24 hours, and

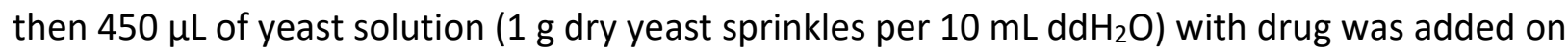
top of the food. Drugged larvae were allowed to develop and adults were scored by genotype daily as they eclosed. Concentrated drug stock solutions were reconstituted as follows: Cisplatin at $1 \mathrm{mg} / \mathrm{mL}$ in phosphate buffered saline (PBS); Mitomycin $\mathrm{C}$ at $0.5 \mathrm{mg} / \mathrm{mL}$ in $\mathrm{ddH}_{2} \mathrm{O}$; Hydroxyurea at $5 \mathrm{mg} / \mathrm{mL}$ in $\mathrm{dd}_{2} \mathrm{O}$.

$\mu-C T$

Control, traip-, and traip- + ubi-GFP::Traip flies were processed for $\mu$-CT according to Schoborg et al., 2019. Briefly, adult flies were aged to 2-4 days old, anesthetized on $\mathrm{CO}_{2}$, de-waxed in PBST (0.5\% Triton X-100), and fixed in Bouin's solution for 24 hours. Fixed flies were washed in $0.1 \mathrm{M} \mathrm{Na}_{2} \mathrm{HPO}_{4}$ with $1.8 \%$ sucrose and then stained in $0.1 \mathrm{~N}$ solution of $\mathrm{I}_{2} \mathrm{KI}$ for 2 days. Stained flies were washed with ultrapure $\mathrm{H}_{2} \mathrm{O}$ and scanned using SkyScan 1172 desktop scanner. Tomograms were generated with NRecon, and 3D volumetric segmentation was performed using Dragonfly. Brain measurements were normalized to thorax width to control for overall size differences among individual flies. 


\section{Immunostaining}

Animals at appropriate stages were collected and aged: adult flies were aged to 3-4 days old unless otherwise stated; white pre-pupae were collected and aged for 24,48 , or 72 hours to obtain pupal stages. Staining procedures were similar to Janelia FlyLight protocols (https://www.janelia.org/project-team/flylight/protocols). Tissues were dissected in SF900 S2 cell media (Fisher Scientific) and fixed with 2\% paraformaldehyde in SF900 for 2 hours at room temperature (RT) or overnight at $4^{\circ} \mathrm{C}$. Fixed samples were washed three times in PBST (PBS + 0.5\% Triton-X100) and blocked for 1.5 hours in PBST + 5\% normal goat serum (NGS). Blocked samples were incubated in PBST + NGS with primary antibody for 1-2 hours at RT and then 1-2 overnights at $4^{\circ} \mathrm{C}$. Primary antibody concentrations were as follows: mouse anti-Fasll (DSHB 1D4) 1:50; rat anti-CadN (DSHB DN-Ex\#8) 1:25; mouse anti-Nrg (DSHB BP104) 1:25; mouse antiElav (DSHB 7E8A 10) 1:100; mouse anti-Lamin (DSHB ADL84.12) 1:100; mouse anti- $p \mathrm{H} 2 \mathrm{Av}$ (DSHB UNC93-5.2.1) 1:100; mouse anti-Tubulin (DSHB E7) 1:200; mouse anti-GFP (Thermo Fisher A11122) 1:1000; mouse anti-Phospho-tyrosine (Millipore 4G10) 1:100; rabbit antiPhospho-histone H3 (Millipore H3S10P) 1:40000; guinea pig anti-Dpn (Skeath lab, Washington University in St. Louis) 1:50. Samples were washed five times in PBST + NGS, and then incubated in PBST + NGS with 1:500 secondary antibody (Thermo Fisher) and 1x DAPI for 1-2 hours at RT and then 1-3 overnights at $4^{\circ} \mathrm{C}$. Samples were washed three times in PBST + NGS and then three times in PBST, before being post-fixed in 4\% paraformaldehyde in PBS for 1 hour at RT. Postfixed samples were washed twice in PBST, and then washed in PBS until all Triton-X100 was removed. Samples were mounted on poly-L-lysine coated \#1.5 coverslips, rinsed in $\mathrm{ddd}_{2} \mathrm{O}$, and then dehydrated through a series of $30 \%, 50 \%, 75 \%, 95 \%$ and then three $100 \%$ ethanol 10 minute baths. Dehydrated coverslips were then bathed three times in $100 \%$ xylene for 5 minutes, before being mounted in DPX on slides with \#1.5 coverslips as spacers. DPX was cured at RT overnight. For phalloidin-stained samples, Phalloidin-Atto 647N (Sigma Aldrich) was added at 1:40 in PBS for 20 minutes after secondary antibody incubation, and samples were not post-fixed and were mounted in AquaPoly Mount (Polysciences, Inc.). 


\section{Light Microscopy}

Most fixed samples were imaged using a Zeiss LSM 880 Confocal Microscope with a 63x/1.4 NA objective, GaAsP detectors, and 405, 488, 561, and $641 \mathrm{~nm}$ laser lines, controlled using Zen Black software. Some fixed samples (Figures 4A, 5A, 5I, 5J) were imaged using a Nikon W1 spinning disc confocal equipped with a Prime BSI cMOS camera (Photometrics) and a 100X/1.4 NA silicon immersion objective, controlled using Nikon Elements software. Live imaging was performed on either using the same Nikon W1 with 100x/1.4 NA silicon or 40x/1.3 NA oil immersion objective, or using an Eclipse Ti2 (Nikon) with a 100X/1.49 NA objective or a 40x/1.3 NA objective and a 1.5X tube lens, an ORCA-Flash 4.0 CMOS camera (Hamamatsu Photonics), and 405, 491, 561, and $642 \mathrm{~nm}$ laser lines, controlled by MetaMorph software. For live imaging, larval brains were dissected in Schneider's media and mounted by sandwiching between a \#1.5 coverslip and a 50-mm lummox dish, with droplets of Halocarbon oil 700 as a cushion and surrounding the edge of the coverslip. ImageJ was used to generate maximum intensity projections for presentation.

Image Analysis

For MB $\alpha$ lobe cross-sectional analysis, image stacks of $\alpha$ lobes were stack rotated to be perpendicular to the viewing plane using the Interactive Stack Rotation plugin in ImageJ. Rotated MB lobes were then cross sectioned through the middle of the lobe using the reslice tool, and the area of the resliced MB lobe was measured in ImageJ. For MB volumetric analysis, either Fasll-positive or OK107-GAL4 > mCD8::GFP-positive MB lobes were segmented and measured using the Pixel Classification and 3D Object Analysis tools in Aivia software. For KC counting analysis, the OK107-GAL4 > NLS::mCherry-positive KCs were similarly segmented and counted using Pixel Classification and 3D Object Analysis tools in Aivia software. $\mathrm{yH}_{2 \mathrm{Av}}$ puncta were thresholded and identified using the 3D Objects Counter in ImageJ. Then, $\mathrm{\gamma H} 2 \mathrm{Av}$ objects inside the nucleus, as marked by anti-lamin immunostaining, were counted in each MB-NB. 


\section{Statistical analysis}

Data analysis was performed using Microsoft Excel and GraphPad Prism. In all graphs, the mean \pm standard deviation, and all individual data points are presented. Sample sizes were primarily determined based on the availability of animals of the proper genotype and developmental stage, and the time required for dissection, processing, and imaging; given these considerations, we processed 8-12 brains per genotype for most experiments, although some brains were not imaged due to physical damage. Experiments measuring MB size were typically performed once, with control and traip ${ }^{\Delta}$ conditions repeated in each experiment. Shapiro-Wilk test was used to test the assumption of normality. Statistical tests used to determine significance include t-test, Mann-Whitney U test, ANOVA with Tukey's test, and chi-squared test, and are reported in Figure legends.

\section{Competing Interests}

The authors declare that no competing interests exist.

\section{Acknowledgements}

We thank the National Heart, Lung, and Blood Institute Light Microscopy Core, especially Xufeng $\mathrm{Wu}$, for support with confocal microscopy. We thank Bloomington Drosophila Stock Center for fly stocks, and Developmental Studies Hybridoma Bank for antibodies. Carey Fagerstrom performed all cloning. Rachel Ng performed the $\mu$-CT experiments. We thank Alex Kelly, Matthew Hannaford, and Ramya Varadarajan for helpful discussion, and Todd Schoborg, Brian Galletta, Ed Giniger, Hong Xu for critically reading the manuscript. This work is supported by the Division of Intramural Research at the NHLBI/NIH (1ZIAHL006126 to N.M.R.). 
bioRxiv preprint doi: https://doi.org/10.1101/2021.07.07.451466; this version posted July 7, 2021. The copyright holder for this preprint (which was not certified by peer review) is the author/funder, who has granted bioRxiv a license to display the preprint in perpetuity. It is made available under aCC-BY-NC-ND 4.0 International license.

\section{Author Contributions}

R.S.O. conceived of the project, designed and performed all experiments, and wrote the manuscript. N.M.R. oversaw the project, designed and discussed experiments, and edited the manuscript. 


\section{References}

Abbott, M.K., Lengyel, J.A., 1991. Embryonic head involution and rotation of male terminalia require the Drosophila locus head involution defective. Genetics 129, 783-789.

Arroyo, M., Kuriyama, R., Trimborn, M., Keifenheim, D., Cañuelo, A., Sánchez, A., Clarke, D.J., Marchal, J.A., 2017. MCPH1, mutated in primary microcephaly, is required for efficient chromosome alignment during mitosis. Sci. Rep. 7, 13019.

Aylon, Y., Oren, M., 2011. p53: guardian of ploidy. Mol. Oncol. 5, 315-323.

Barrera, J.A., Kao, L.-R., Hammer, R.E., Seemann, J., Fuchs, J.L., Megraw, T.L., 2010. CDK5RAP2 regulates centriole engagement and cohesion in mice. Dev. Cell 18, 913-926.

Bianchi, F.T., Berto, G.E., Di Cunto, F., 2018. Impact of DNA repair and stability defects on cortical development. Cell Mol. Life Sci. 75, 3963-3976.

Bianchi, V., Pontis, E., Reichard, P., 1986. Changes of deoxyribonucleoside triphosphate pools induced by hydroxyurea and their relation to DNA synthesis. J. Biol. Chem. 261, 1603716042.

Deans, A.J., West, S.C., 2011. DNA interstrand crosslink repair and cancer. Nat. Rev. Cancer 11, 467-480.

Deng, L., Wu, R.A., Sonneville, R., Kochenova, O.V., Labib, K., Pellman, D., Walter, J.C., 2019. Mitotic CDK promotes replisome disassembly, fork breakage, and complex DNA rearrangements. Mol. Cell 73, 915-929.e6.

Feng, W., Guo, Y., Huang, J., Deng, Y., Zang, J., Huen, M.S.-Y., 2016. TRAIP regulates replication fork recovery and progression via PCNA. Cell Discov. 2, 16016.

Gisselsson, D., Björk, J., Höglund, M., Mertens, F., Dal Cin, P., Akerman, M., Mandahl, N., 2001. Abnormal nuclear shape in solid tumors reflects mitotic instability. Am. J. Pathol. 158, 199-206. 
Gregg, R.W., Molepo, J.M., Monpetit, V.J., Mikael, N.Z., Redmond, D., Gadia, M., Stewart, D.J., 1992. Cisplatin neurotoxicity: the relationship between dosage, time, and platinum concentration in neurologic tissues, and morphologic evidence of toxicity. J. Clin. Oncol. 10, 795-803.

Han, Y.G., Yun, M., Choi, M., Lee, S.-G., Kim, H., 2019. TRAIP regulates Histone H2B monoubiquitination in DNA damage response pathways. Oncol. Rep. 41, 3305-3312.

Harley, M.E., Murina, O., Leitch, A., Higgs, M.R., Bicknell, L.S., Yigit, G., Blackford, A.N., Zlatanou, A., Mackenzie, K.J., Reddy, K., Halachev, M., McGlasson, S., Reijns, M.A.M., Fluteau, A., Martin, C.-A., Sabbioneda, S., Elcioglu, N.H., Altmüller, J., Thiele, H., Greenhalgh, L., Chessa, L., Maghnie, M., Salim, M., Bober, M.B., Nürnberg, P., Jackson, S.P., Hurles, M.E., Wollnik, B., Stewart, G.S., Jackson, A.P., 2016. TRAIP promotes DNA damage response during genome replication and is mutated in primordial dwarfism. Nat. Genet. 48, 3643.

Haynie, J.L., Bryant, P.J., 1977. The effects of X-rays on the proliferation dynamics of cells in the imaginal wing disc of Drosophila melanogaster. Wilhelm Roux'. Archiv. 183, 85-100.

Heddle, J.A., Carrano, A.V., 1977. The DNA content of micronuclei induced in mouse bone marrow by gamma-irradiation: evidence that micronuclei arise from acentric chromosomal fragments. Mutat. Res. 44, 63-69.

Heisenberg, M., 2003. Mushroom body memoir: from maps to models. Nat. Rev. Neurosci. 4, 266-275.

Heisenberg, M., Heusipp, M., Wanke, C., 1995. Structural plasticity in the Drosophila brain. J. Neurosci. 15, 1951-1960.

Hoffmann, S., Smedegaard, S., Nakamura, K., Mortuza, G.B., Räschle, M., Ibañez de Opakua, A., Oka, Y., Feng, Y., Blanco, F.J., Mann, M., Montoya, G., Groth, A., Bekker-Jensen, S., Mailand, N., 2016. TRAIP is a PCNA-binding ubiquitin ligase that protects genome stability after replication stress. J. Cell Biol. 212, 63-75. 
Hussain, M.S., Baig, S.M., Neumann, S., Nürnberg, G., Farooq, M., Ahmad, I., Alef, T., Hennies, H.C., Technau, M., Altmüller, J., Frommolt, P., Thiele, H., Noegel, A.A., Nürnberg, P., 2012. A truncating mutation of CEP135 causes primary microcephaly and disturbed centrosomal function. Am. J. Hum. Genet. 90, 871-878.

Ito, K., Hotta, Y., 1992. Proliferation pattern of postembryonic neuroblasts in the brain of Drosophila melanogaster. Dev. Biol. 149, 134-148.

Jayaraman, D., Bae, B.-I., Walsh, C.A., 2018. The genetics of primary microcephaly. Annu. Rev. Genomics. Hum. Genet. 19, 177-200.

Jeffers, L.J., Coull, B.J., Stack, S.J., Morrison, C.G., 2008. Distinct BRCT domains in Mcph1/Brit1 mediate ionizing radiation-induced focus formation and centrosomal localization. Oncogene 27, 139-144.

Khetarpal, P., Das, S., Panigrahi, I., Munshi, A., 2016. Primordial dwarfism: overview of clinical and genetic aspects. Mol. Genet. Genomics 291, 1-15.

Larsen, N.B., Gao, A.O., Sparks, J.L., Gallina, I., Wu, R.A., Mann, M., Räschle, M., Walter, J.C., Duxin, J.P., 2019. Replication-Coupled DNA-Protein Crosslink Repair by SPRTN and the Proteasome in Xenopus Egg Extracts. Mol. Cell 73, 574-588.e7.

Liu, Y., Nielsen, C.F., Yao, Q., Hickson, I.D., 2014. The origins and processing of ultra fine anaphase DNA bridges. Curr. Opin. Genet. Dev. 26, 1-5.

Merkle, J.A., Rickmyre, J.L., Garg, A., Loggins, E.B., Jodoin, J.N., Lee, E., Wu, L.P., Lee, L.A., 2009. no poles encodes a predicted E3 ubiquitin ligase required for early embryonic development of Drosophila. Development 136, 449-459.

Modi, M.N., Shuai, Y., Turner, G.C., 2020. The drosophila mushroom body: from architecture to algorithm in a learning circuit. Annu. Rev. Neurosci. 43, 465-484.

Naim, V., Rosselli, F., 2009. The FANC pathway and BLM collaborate during mitosis to prevent micro-nucleation and chromosome abnormalities. Nat. Cell Biol. 11, 761-768. 
Nandakumar, S., Grushko, O., Buttitta, L.A., 2020. Polyploidy in the adult Drosophila brain. Elife 9.

Nano, M., Basto, R., 2017. Consequences of centrosome dysfunction during brain development. Adv. Exp. Med. Biol. 1002, 19-45.

Nano, M., Gemble, S., Simon, A., Pennetier, C., Fraisier, V., Marthiens, V., Basto, R., 2019. CellCycle Asynchrony Generates DNA Damage at Mitotic Entry in Polyploid Cells. Curr. Biol. 29, 3937-3945.e7.

Pampalona, J., Frías, C., Genescà, A., Tusell, L., 2012. Progressive telomere dysfunction causes cytokinesis failure and leads to the accumulation of polyploid cells. PLoS Genet. 8, e1002679.

Park, E.-S., Choi, S., Kim, J.-M., Jeong, Y., Choe, J., Park, C.-S., Choi, Y., Rho, J., 2007. Early embryonic lethality caused by targeted disruption of the TRAF-interacting protein (TRIP) gene. Biochem. Biophys. Res. Commun. 363, 971-977.

Parks, A.L., Cook, K.R., Belvin, M., Dompe, N.A., Fawcett, R., Huppert, K., Tan, L.R., Winter, C.G., Bogart, K.P., Deal, J.E., Deal-Herr, M.E., Grant, D., Marcinko, M., Miyazaki, W.Y., Robertson, S., Shaw, K.J., Tabios, M., Vysotskaia, V., Zhao, L., Andrade, R.S., Edgar, K.A., Howie, E., Killpack, K., Milash, B., Norton, A., Thao, D., Whittaker, K., Winner, M.A., Friedman, L., Margolis, J., Singer, M.A., Kopczynski, C., Curtis, D., Kaufman, T.C., Plowman, G.D., Duyk, G., Francis-Lang, H.L., 2004. Systematic generation of highresolution deletion coverage of the Drosophila melanogaster genome. Nat. Genet. 36, 288-292.

Passemard, S., Kaindl, A.M., Verloes, A., 2013. Microcephaly. Handb Clin Neurol 111, 129-141.

Peterson, S.E., Yang, A.H., Bushman, D.M., Westra, J.W., Yung, Y.C., Barral, S., Mutoh, T., Rehen, S.K., Chun, J., 2012. Aneuploid cells are differentially susceptible to caspase-mediated death during embryonic cerebral cortical development. J. Neurosci. 32, 16213-16222. 
Pfau, S.J., Silberman, R.E., Knouse, K.A., Amon, A., 2016. Aneuploidy impairs hematopoietic stem cell fitness and is selected against in regenerating tissues in vivo. Genes Dev. 30, 1395-1408.

Poulton, J.S., Cuningham, J.C., Peifer, M., 2017. Centrosome and spindle assembly checkpoint loss leads to neural apoptosis and reduced brain size. J. Cell Biol. 216, 1255-1265.

Priego Moreno, S., Jones, R.M., Poovathumkadavil, D., Scaramuzza, S., Gambus, A., 2019. Mitotic replisome disassembly depends on TRAIP ubiquitin ligase activity. Life Sci. Alliance 2.

Rai, R., Phadnis, A., Haralkar, S., Badwe, R.A., Dai, H., Li, K., Lin, S.-Y., 2008. Differential regulation of centrosome integrity by DNA damage response proteins. Cell Cycle 7, 2225-2233.

Reddy, M.V., Randerath, K., 1987. 32P-analysis of DNA adducts in somatic and reproductive tissues of rats treated with the anticancer antibiotic, mitomycin C. Mutat. Res. 179, 7588.

Rehen, S.K., McConnell, M.J., Kaushal, D., Kingsbury, M.A., Yang, A.H., Chun, J., 2001. Chromosomal variation in neurons of the developing and adult mammalian nervous system. Proc. Natl. Acad. Sci. USA 98, 13361-13366.

Rickmyre, J.L., Dasgupta, S., Ooi, D.L.-Y., Keel, J., Lee, E., Kirschner, M.W., Waddell, S., Lee, L.A., 2007. The Drosophila homolog of MCPH1, a human microcephaly gene, is required for genomic stability in the early embryo. J. Cell Sci. 120, 3565-3577.

Royou, A., Gagou, M.E., Karess, R., Sullivan, W., 2010. BubR1- and Polo-coated DNA tethers facilitate poleward segregation of acentric chromatids. Cell 140, 235-245.

Schoborg, T.A., Smith, S.L., Smith, L.N., Morris, H.D., Rusan, N.M., 2019. Micro-computed tomography as a platform for exploring Drosophila development. Development 146. 
Shi, L., Qalieh, A., Lam, M.M., Keil, J.M., Kwan, K.Y., 2019. Robust elimination of genomedamaged cells safeguards against brain somatic aneuploidy following Knl1 deletion. Nat. Commun. 10, 2588.

Shi, Q., King, R.W., 2005. Chromosome nondisjunction yields tetraploid rather than aneuploid cells in human cell lines. Nature 437, 1038-1042.

Sonneville, R., Bhowmick, R., Hoffmann, S., Mailand, N., Hickson, I.D., Labib, K., 2019. TRAIP drives replisome disassembly and mitotic DNA repair synthesis at sites of incomplete DNA replication. Elife 8.

Soo Lee, N., Jin Chung, H., Kim, H.-J., Yun Lee, S., Ji, J.-H., Seo, Y., Hun Han, S., Choi, M., Yun, M., Lee, S.-G., Myung, K., Kim, Y., Chul Kang, H., Kim, H., 2016. TRAIP/RNF206 is required for recruitment of RAP80 to sites of DNA damage. Nat. Commun. 7, 10463.

Storchova, Z., Kuffer, C., 2008. The consequences of tetraploidy and aneuploidy. J. Cell Sci. 121, 3859-3866.

Swider, Z.T., Ng, R.K., Varadarajan, R., Fagerstrom, C.J., Rusan, N.M., 2019. Fascetto (PRC1) interacting protein (FIP) ensures proper cytokinesis and ploidy. Mol. Biol. Cell mbcE18090573.

Truman, J.W., Bate, M., 1988. Spatial and temporal patterns of neurogenesis in the central nervous system of Drosophila melanogaster. Dev. Biol. 125, 145-157.

Villa, F., Fujisawa, R., Ainsworth, J., Nishimura, K., Lie-A-Ling, M., Lacaud, G., Labib, K.P., 2021. CUL2LRR1, TRAIP and p97 control CMG helicase disassembly in the mammalian cell cycle. EMBO Rep. e52164.

Wu, R.A., Pellman, D.S., Walter, J.C., 2020. The Ubiquitin Ligase TRAIP: Double-Edged Sword at the Replisome. Trends Cell Biol. Feb;31(2):75-85.

Wu, R.A., Semlow, D.R., Kamimae-Lanning, A.N., Kochenova, O.V., Chistol, G., Hodskinson, M.R., Amunugama, R., Sparks, J.L., Wang, M., Deng, L., Mimoso, C.A., Low, E., Patel, K.J., 
bioRxiv preprint doi: https://doi.org/10.1101/2021.07.07.451466; this version posted July 7, 2021. The copyright holder for this preprint (which was not certified by peer review) is the author/funder, who has granted bioRxiv a license to display the preprint in perpetuity. It is made available under aCC-BY-NC-ND 4.0 International license.

Walter, J.C., 2019. TRAIP is a master regulator of DNA interstrand crosslink repair.

Nature 567, 267-272. 


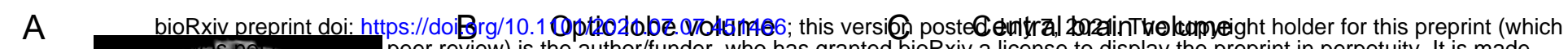

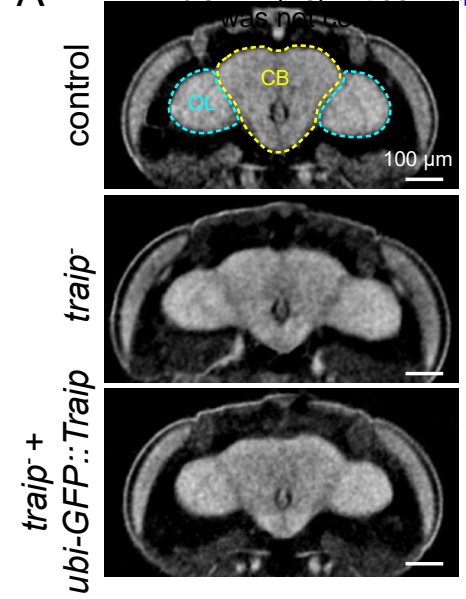
peer review) is the author/funder, who has granted bioRxiv a license to display the preprint in perpetuity. It is made availablesunder aCC-BY-NC-ND 4.0 International licesise.
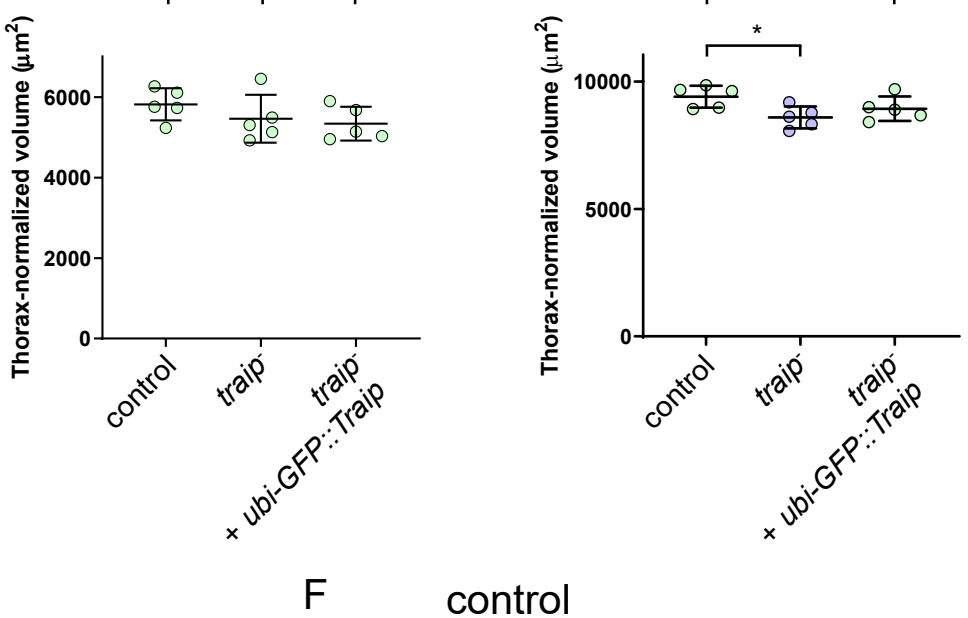

D
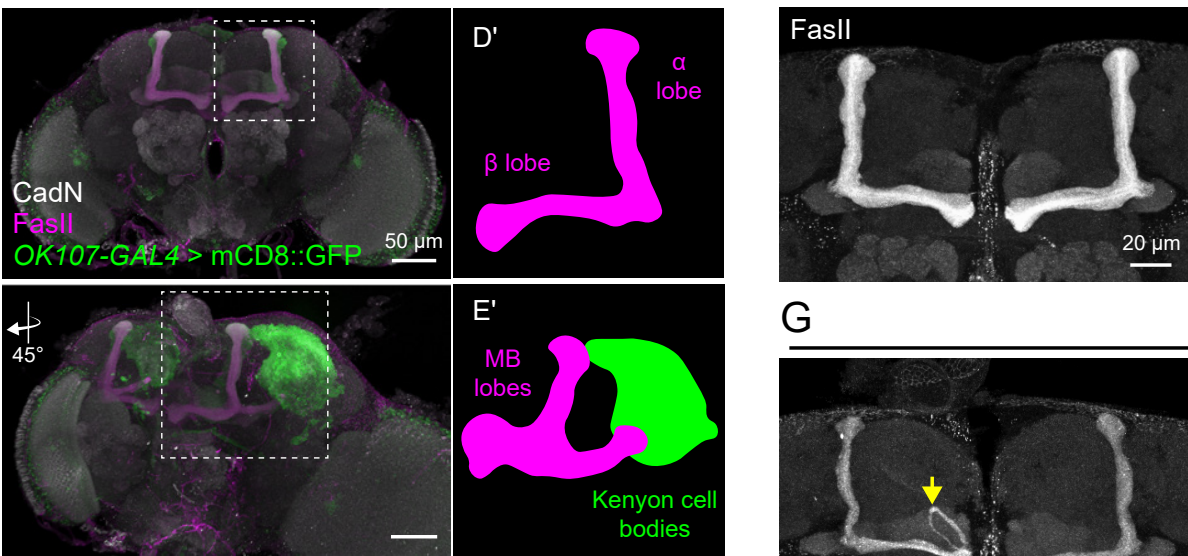

G

traip
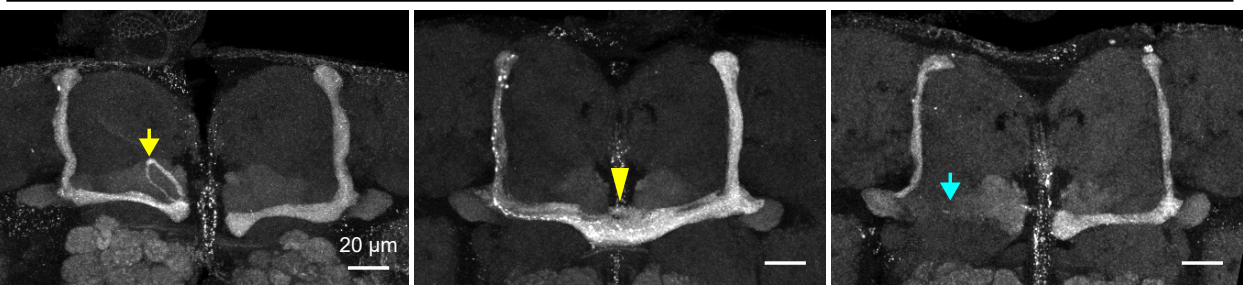

$\mathrm{H}$

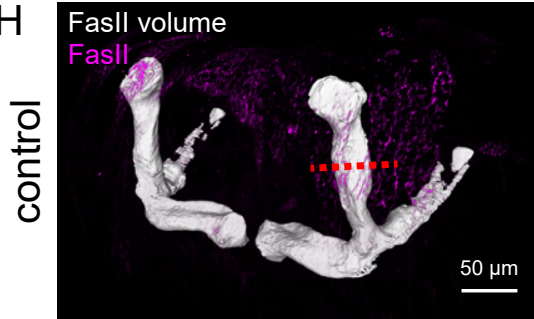

$\frac{\sqrt{3}}{2}$

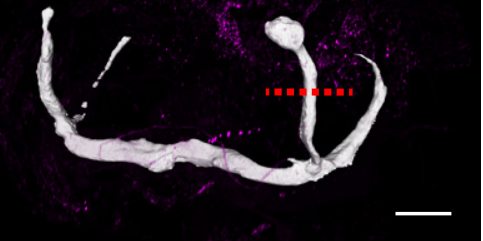

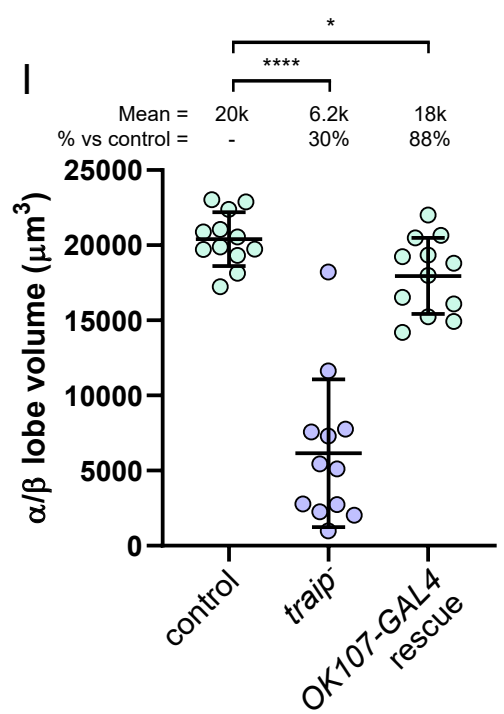
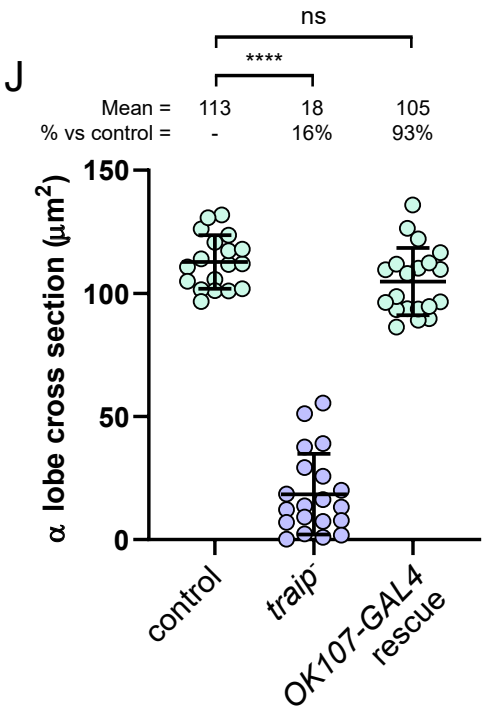

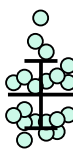

$0^{\circ}$

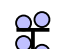

के

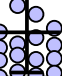

$\mathrm{K}$

traip + rescue OK107-GAL4 > GFP::Traip

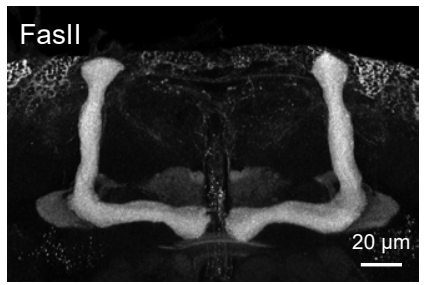

control

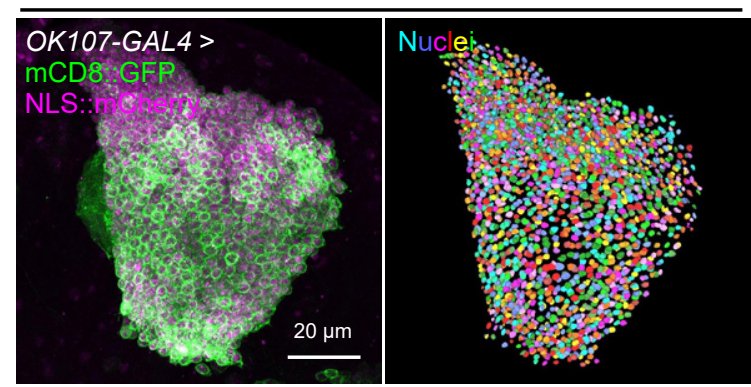

traip

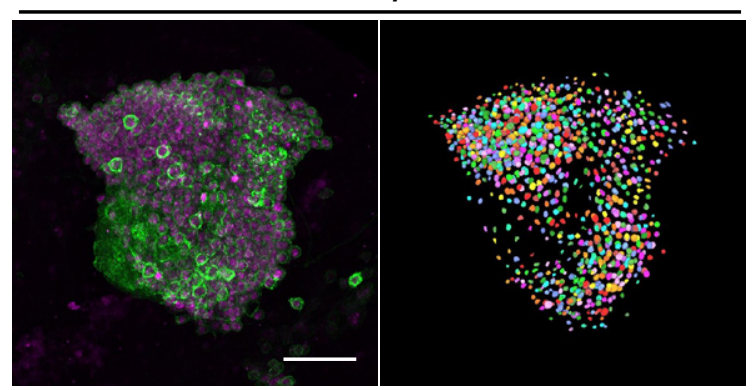

Figure 1 - Traip is required for proper Mushroom Body structure 


\section{Figure 1 - Traip is required for proper Mushroom Body structure}

(A) $\mu$-CT tomograms of control, traip', and traip ${ }^{-}+$ubi-GFP::Traip adult brains. Optic lobes (OL) and central brain $(\mathrm{CB})$ are highlighted on the control.

(B) Thorax-normalized optic lobe volumes. $\mathrm{N}=5$ brains.

(C) Thorax-normalized central brain volumes. $\mathrm{N}=5$ brains; * $\mathrm{p}=0.025$.

(D) Maximum projection of adult brain stained for Fasll ( $\alpha / \beta$ lobes, magenta; cartoon in $\left.D^{\prime}\right)$ and CadN (neuropil, white) and labeled with OK107-GAL4 > mCD8::GFP (green).

(E) Oblique full brain volume projection stained for Fasll, CadN, and labeled with OK107-GAL4 > mCD8::GFP, showing KC bodies on the ventral posterior side projecting axons anteriorly to form the MB lobes (cartoon in $\mathrm{E}^{\prime}$ ).

(F) Control MBs stained for Fasll with stereotypic L-shaped $\alpha / \beta$ lobes.

(G) traip ${ }^{-M B s}$ with reduced size, misguided axon tracts (yellow arrow, $50 \%$ traip vs $0 \%$ control), midline fused MBs (yellow arrowhead, 93\% traip- vs $14 \%$ control), and missing $\alpha$ or $\beta$ lobes (cyan arrow, $29 \%$ traip vs $0 \%$ control).

(H) Control and traip $\alpha / \beta$ lobe volumes (white) segmented from Fasll staining (magenta). Red lines show position of cross-section measurements.

(I) $\alpha / \beta$ lobe volume measurements. $N \geq 12$ MBs. * $p=0.0145$.

(J) $\alpha$ lobe cross-section measurements. $N \geq 18 \mathrm{MBs}$.

(K) MBs from traip + OK107-GAL4 > GFP::Traip rescue with wild-type morphology.

(L) Machine learning segmentation of KCs. OK107-GAL4 > mCD8::GFP (green) + NLS::mCherry (magenta, left panels) were used for $\mathrm{KC}$ nuclei segmentation and counting (multi-color, right panels) of controls and traip.

(M) $\mathrm{KC}$ numbers per hemisphere. $\mathrm{KC}$ are reduced by $60 \%$ in traip ${ }^{-} \mathrm{N}=20$ hemispheres.

In all graphs, bars are mean \pm standard deviation. Two-tailed t-test $(B, C)$ and Mann-Whitney test (I, J, M) were used for significance. ns = not significant, $* * * *<0.0001$. Scale bars $=100 \mu \mathrm{m}(A), 50 \mu \mathrm{m}(\mathrm{D}, \mathrm{E}, \mathrm{H})$, $20 \mu \mathrm{m}(\mathrm{F}, \mathrm{G}, \mathrm{K}, \mathrm{L})$. 


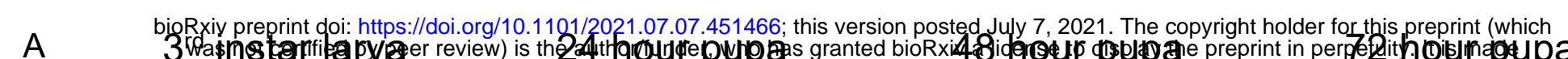

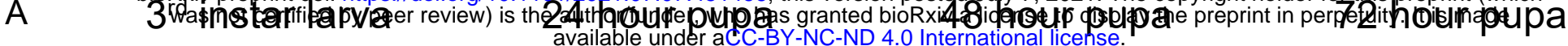

을

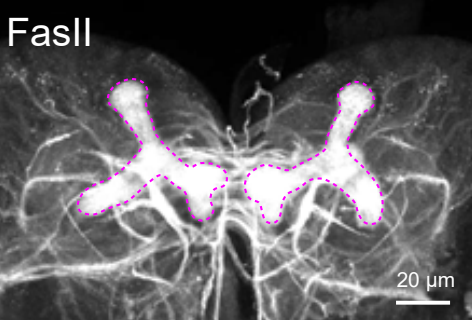

$\frac{\pi}{\pi}$

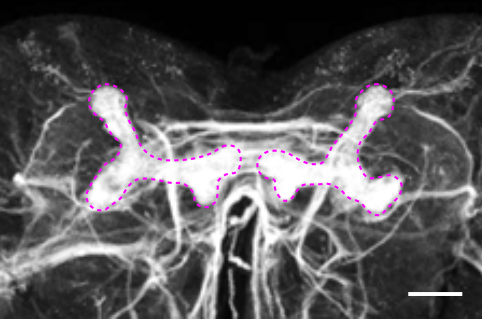

ns

B

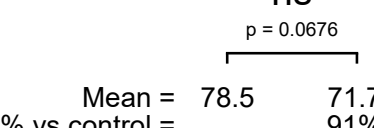

$\%$ vs control $=-78.5 \quad 91 \%$

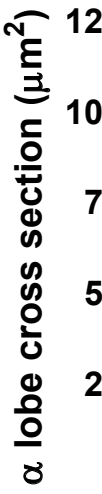

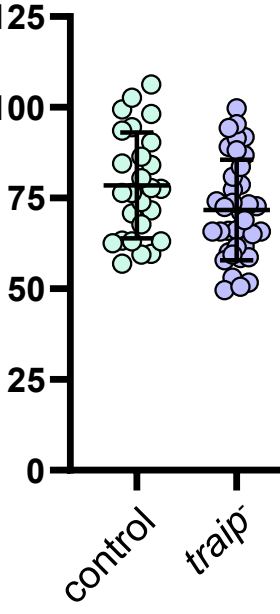

$\overline{3^{\text {rd }} \text { instar }}$

C

3 days old

은

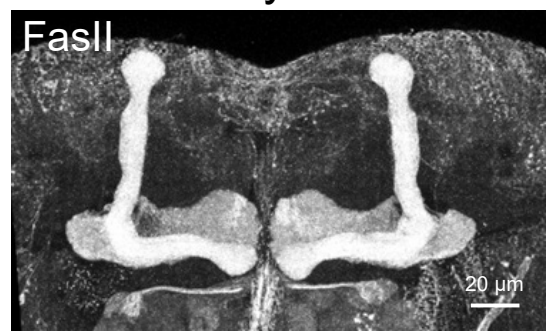

$\frac{\pi}{+\infty}$
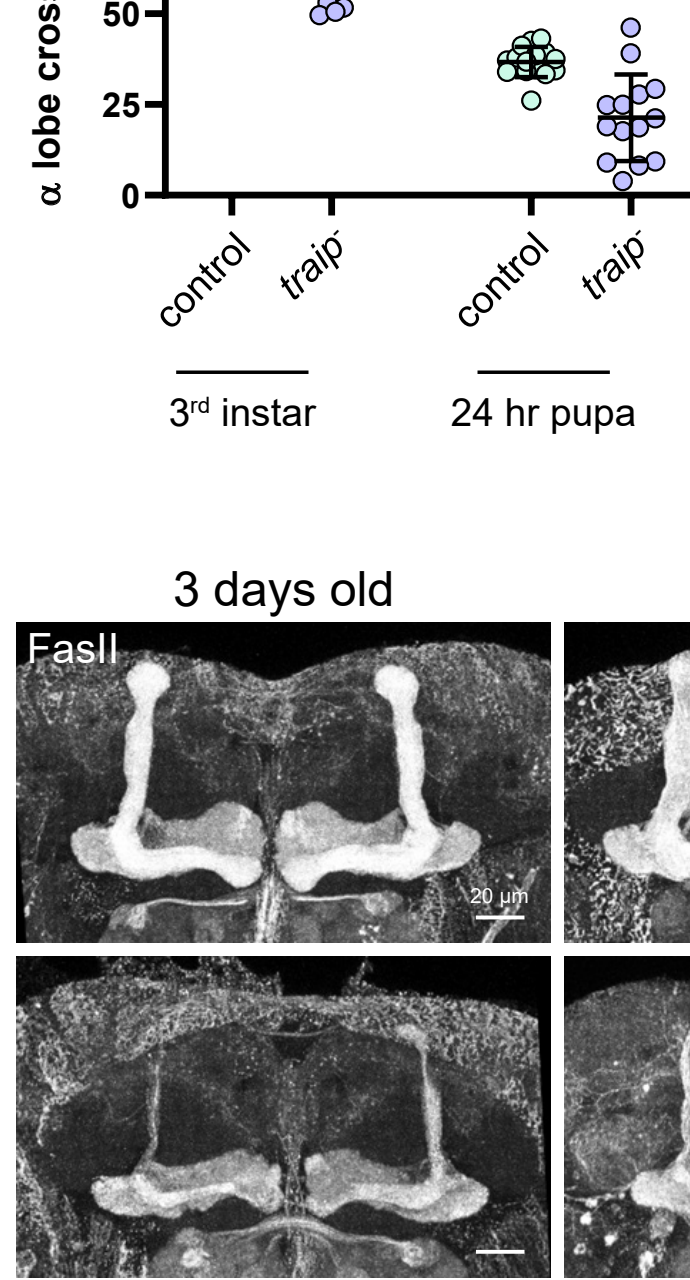
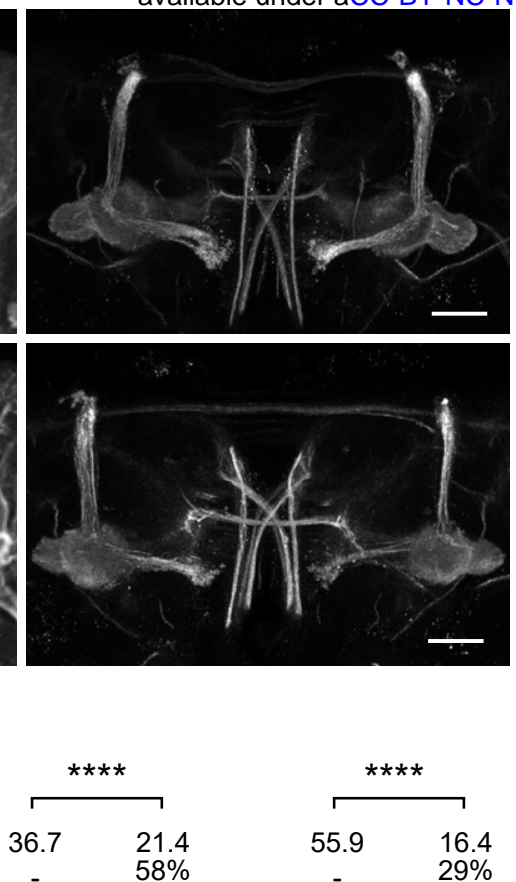

$29 \%$
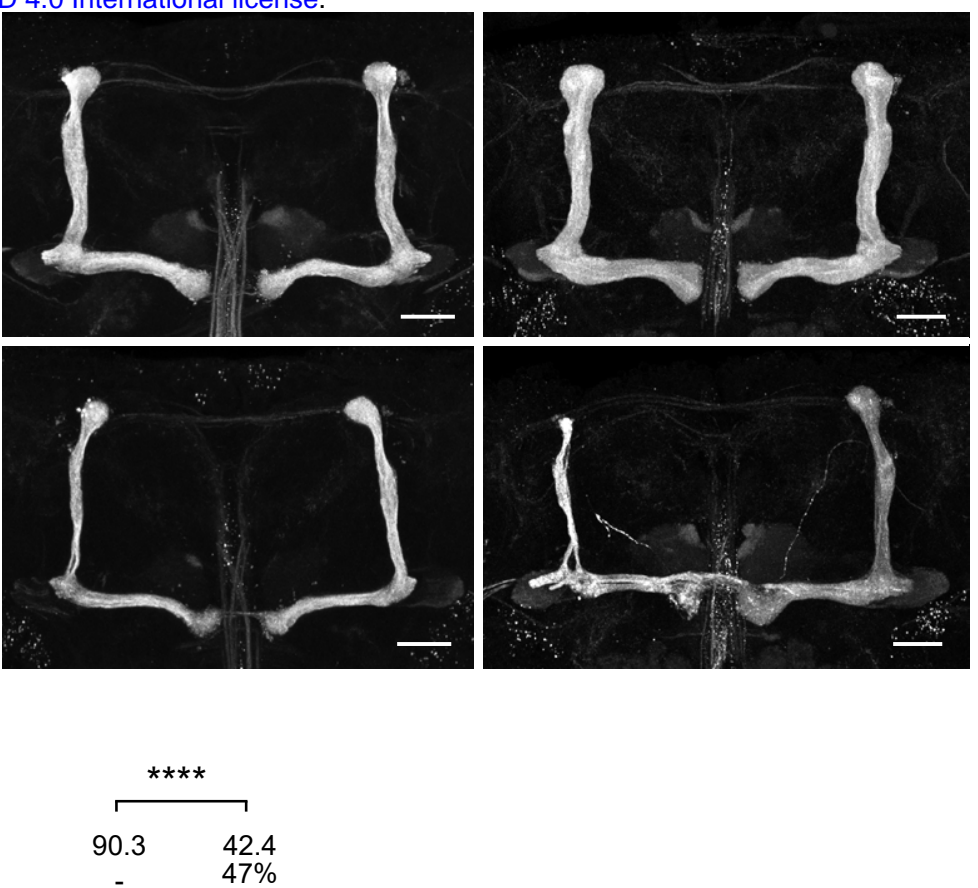

$\frac{0}{8}$
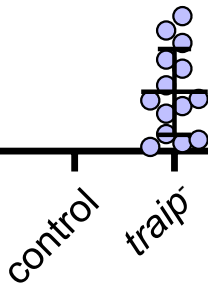

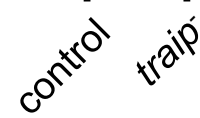

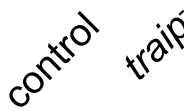

72 hr pupa

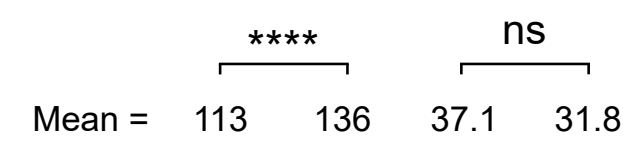

D

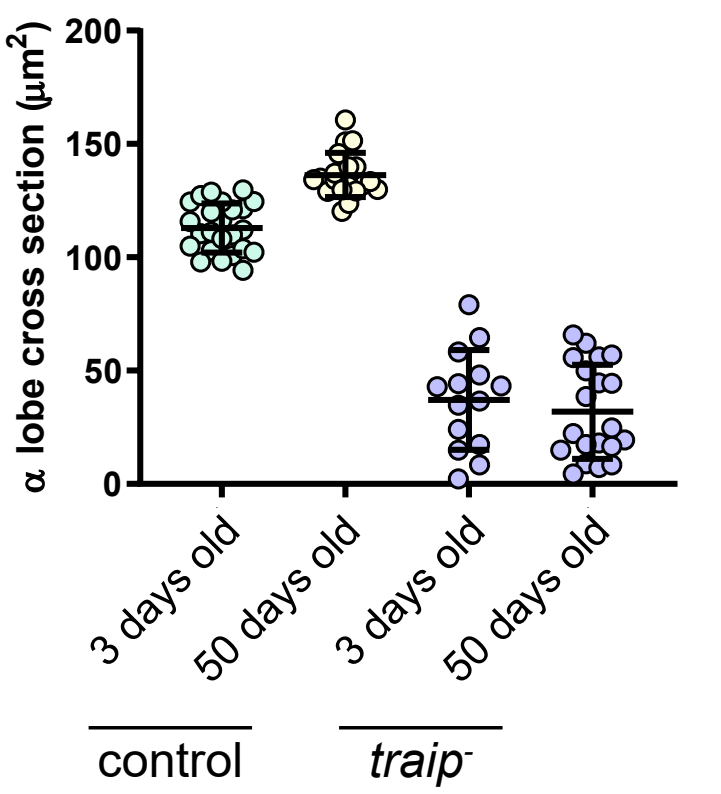

Figure 2 - Traip is required for MB development 


\section{Figure 2 - Traip is required for MB development}

(A) Control (top row) and traip- (bottom row) MBs from (left to right) $3^{\text {rd }}$ instar larvae, 24 hours APF, 48 hours APF, and 72 hours APF pupae stained for Fasll. $3^{\text {rd }}$ instar larval MBs are highlighted in magenta; note that in larval stage, Fasll labels the $\gamma$ lobes, and $\alpha / \beta$ lobes are yet to be born.

(B) MB lobe cross-section measurements of control and traip developmental stages. $3^{\text {rd }}$ instar larval MBs of traip are not significantly reduced compared to controls. In all pupal stages, traip are significantly reduced compared to controls. Note that 24 hours APF MBs appear smaller than larval MBs in part due to extensive morphological remodelling in early metamorphosis (Lee et al., 1999). $N \geq 14$ MBs.

(C) Control (top row) and traip (bottom row) MBs from 3 day old adults (left column) and 50 day old adults (right column) stained for Fasll.

(D) $\alpha$ lobe cross-section measurements show that control MBs increase in size with age, whereas traip MBs do not change. $N \geq 14$ MBs.

Two-tailed t-test was used for significance. $\mathrm{ns}=$ not significant, $* * * * p<0.0001$. Scale bars $=20 \mu \mathrm{m}$. 
bioRxiv preprint doi: https://doi.org/10.1101/2021.07.07.451466; this version posted July 7, 2021. The copyright holder for this preprint (which

A

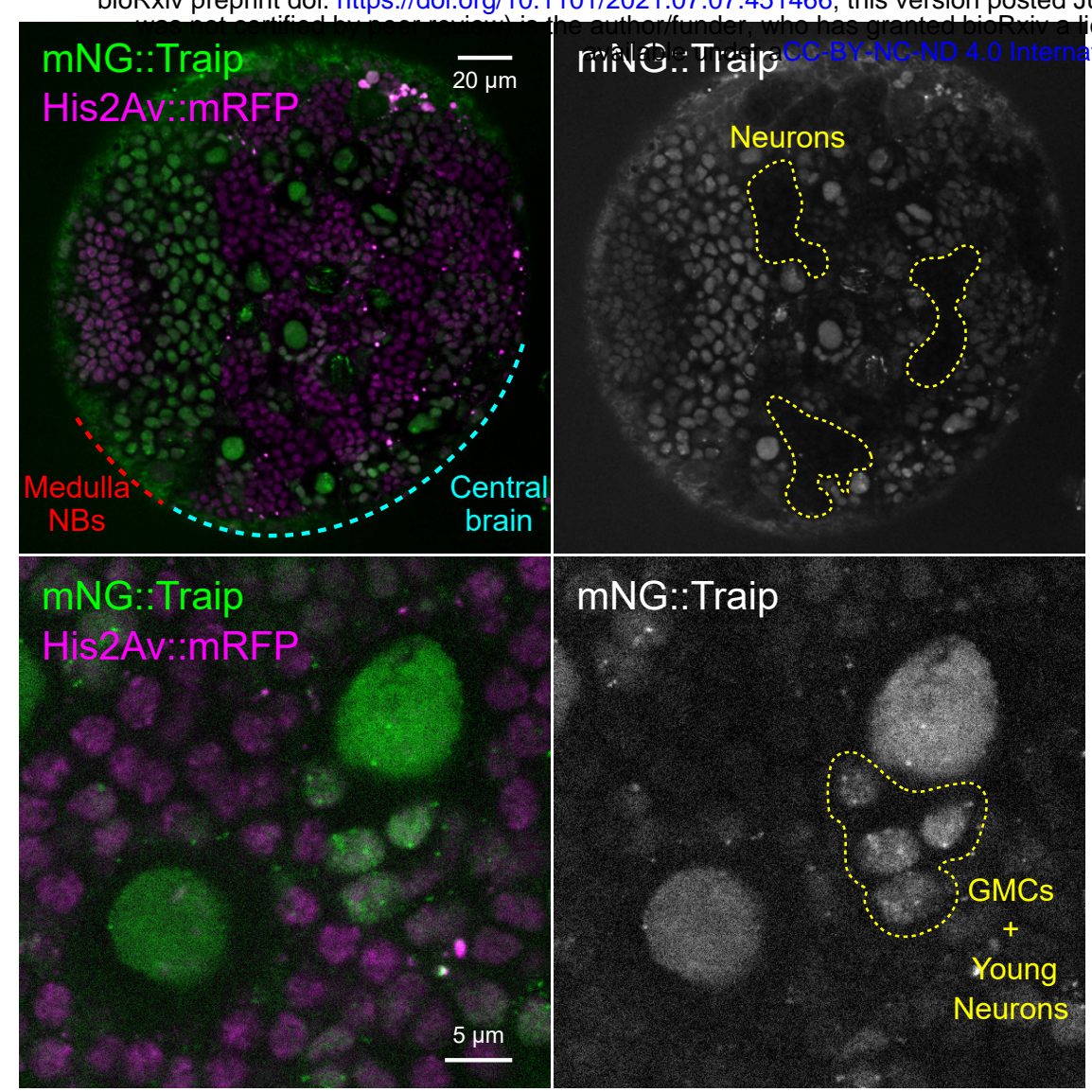

C
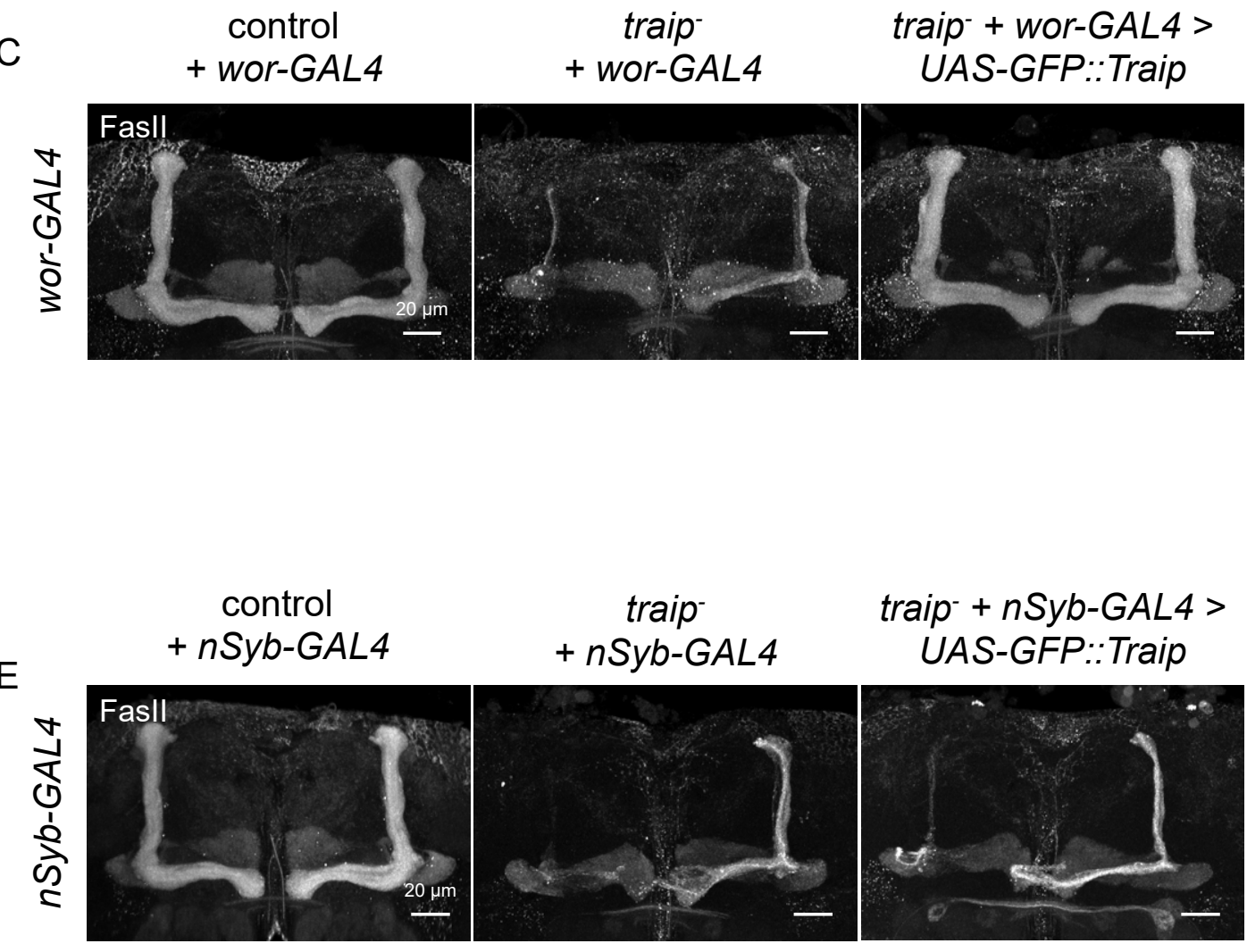
tional license. icense to display the preprint in perpetuity. It is made 


\section{Figure 3 - Traip is required in neuroblasts}

(A) Endogenously tagged mNG::Traip (green, gray) and His2Av::mRFP (magenta) in the $3^{\text {rd }}$ instar larval brain. mNG::Traip is highly expressed in the proliferating cells of the medulla (red zone) and central brain regions (cyan zone), but is absent from areas dominated by neurons (yellow outlines). Cell types were determined by position and morphology as mNG::Traip fluorescence does not survive fixation.

(B) High resolution imaging of $\mathrm{mNG}::$ Traip localized to the nuclei of interphase CB-NBs, and persisting in daughter GMCs and young neurons (yellow outline).

(C) MBs from control, traip", and UAS-GFP::Traip rescue, all with wor-GAL4, stained for Fasll.

(D) $\alpha$ lobe cross-section measurements show that traip ${ }^{-}+$wor-GAL4 > GFP::Traip rescue have wild-type MB size. $\mathrm{N} \geq 22 \mathrm{MBs}$.

(E) MBs from control, traip', and UAS-GFP::Traip rescue, all with nSyb-GAL4, stained for Fasll.

(F) $\alpha$ lobe cross-section measurements show that traip $+n S y b-G A L 4>$ GFP::Traip have reduced MB size. $\mathrm{N} \geq 6 \mathrm{MBs}$.

Two-tailed t-test was used for significance. $n s=$ not significant, $* * * * p<0.0001$. Scale bars $=20 \mu \mathrm{m}(\mathrm{A}$, C, E), $5 \mu \mathrm{m}(\mathrm{B})$. 


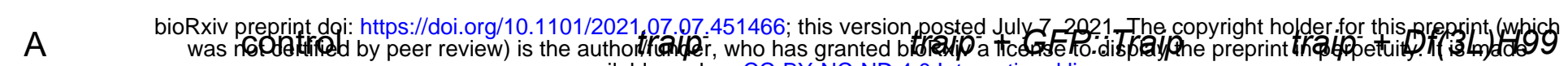

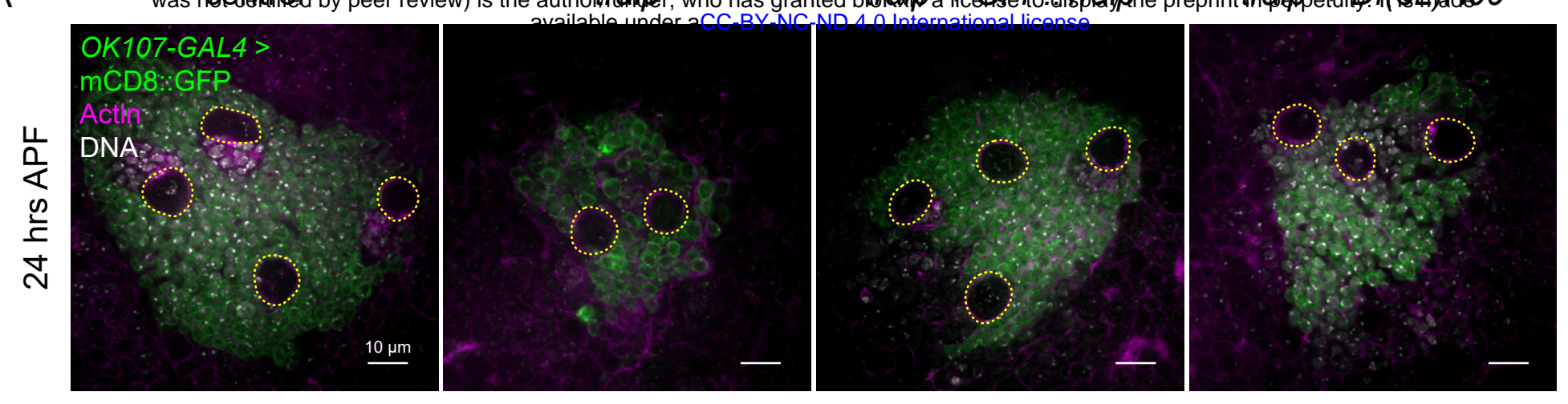

B
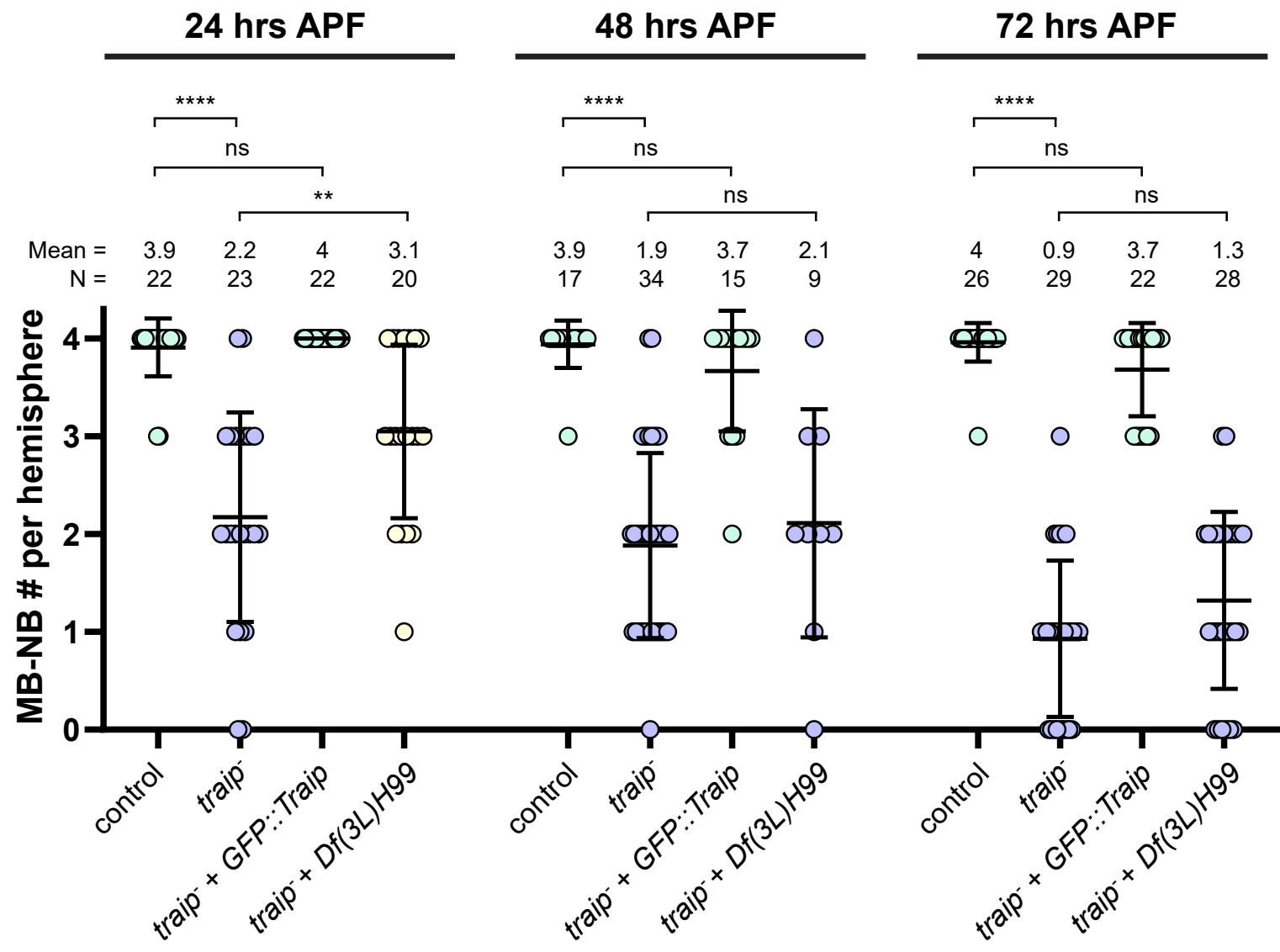

C
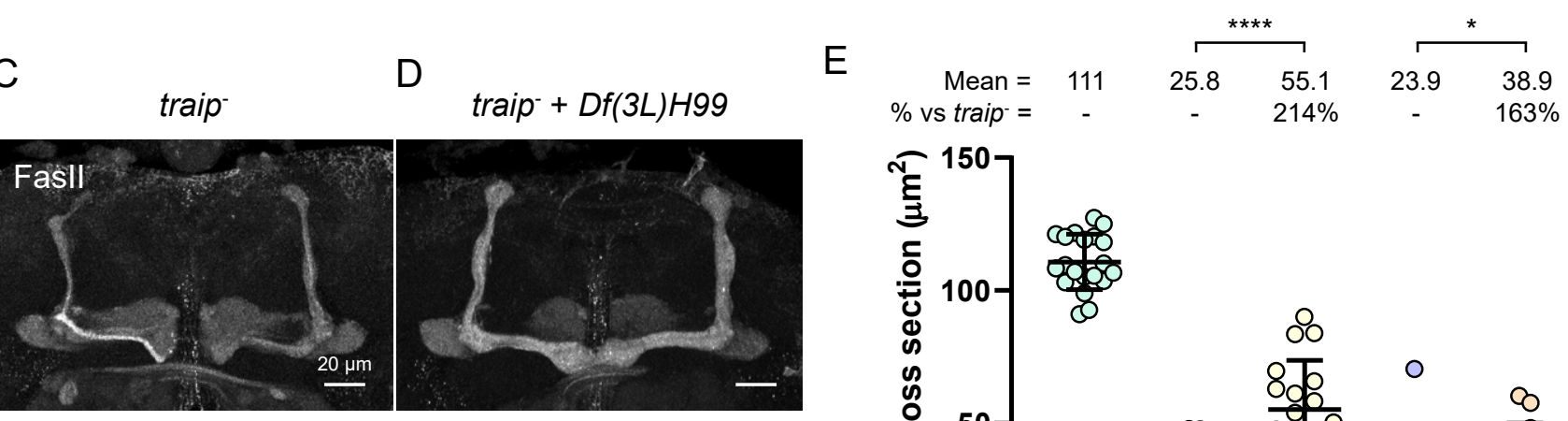

$F$

traip $^{-}+$Drice RNAi
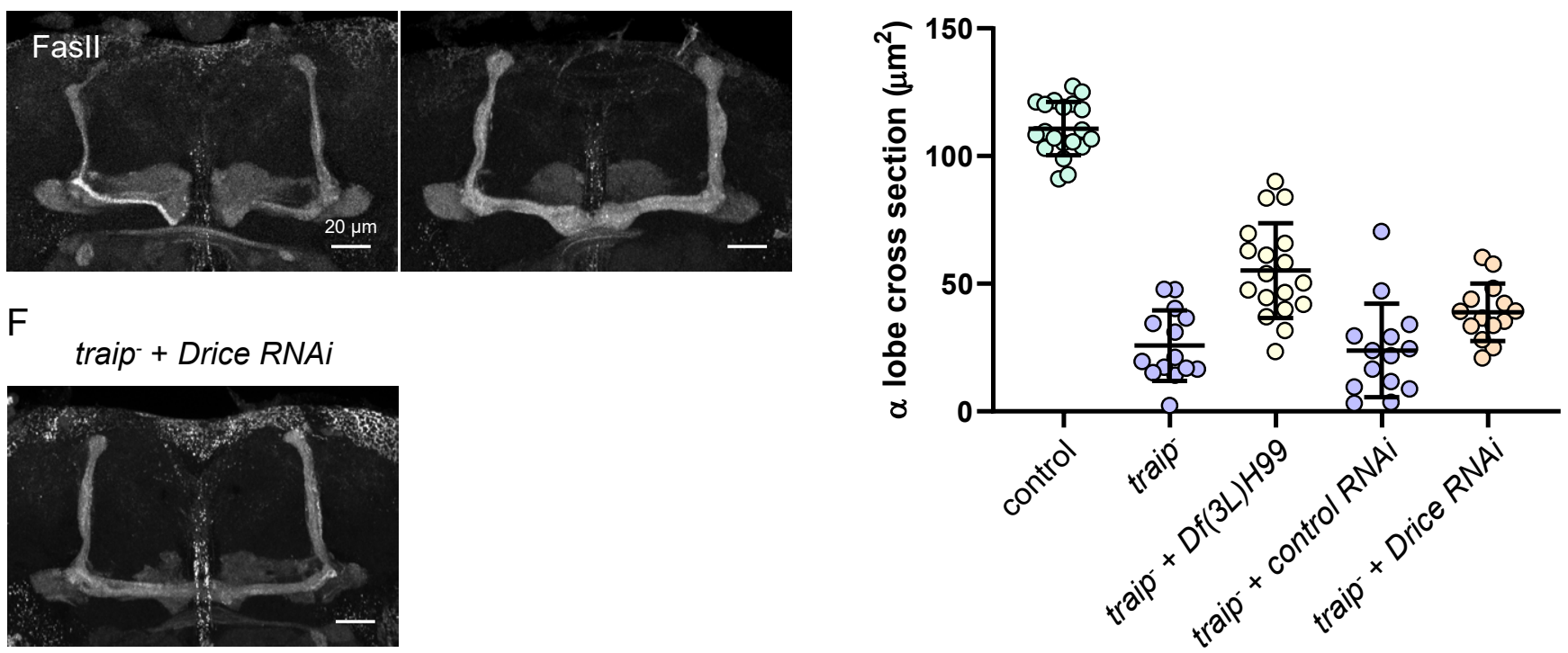

Figure 4 - Traip suppresses MB-NB cell death 


\section{Figure 4 - Traip suppresses MB-NB cell death}

(A) Fields of KCs and MB-NBs labelled with OK107-GAL4 > mCD8::GFP (green), phalloidin (magenta), and DAPI (gray) from (left to right) control, traip" traip $^{-}+$OK107-GAL4 > GFP::Traip rescue, and traip $^{-}+$ $D f(3 L) H 9924$ hours APF pupae. NBs are highlighted in yellow. Scale bars $=10 \mu \mathrm{m}$.

(B) MB-NB number per brain hemisphere for control, traip ${ }^{-}$, traip ${ }^{-}+$GFP::Traip rescue, and traip ${ }^{-}+$ $D f(3 L) H 99$ at 24, 48, and 72 hours APF pupal stages. Two-tailed Mann-Whitney test was used to determine significance. $\mathrm{ns}=$ not significant, ${ }^{* *} \mathrm{p}=0.0069, * * * \mathrm{p}<0.0001 . \mathrm{N}$ is reported above each column.

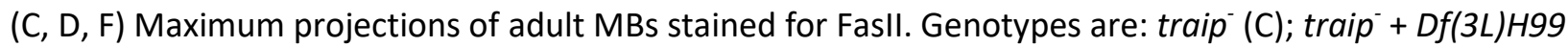
(D); traip + + OK107-GAL4 > Drice RNAi (F). Scale bars $=20 \mu \mathrm{m}$.

(E) $\alpha$ lobe cross-section measurements of control, traip ${ }^{-}$traip $^{-}+D f\left(3 L^{\prime}\right) H_{99}$, traip ${ }^{-}+$control RNAi and traip + Drice RNAi. Two-tailed t-test was used for significance. ${ }^{*} p=0.0149, * * * * p<0.0001 . N \geq 14$ MBs. 


\section{Figure 5 - Traip suppresses multinuclear phenotypes and mitotic DNA bridges}

(A-F) MB-NBs from 24 hours APF pupal brains labelled with OK107-GAL4 > mCD8::GFP (green) and stained for Lamin (red), DNA (gray), and phospho-Tyrosine (blue).

(A) $100 \%(110 / 110)$ of control MB-NBs have relatively smooth, spherical nuclear laminar morphology and no additional nuclear defects.

(B) 11\% (16/140) of traip 24 hours APF MB-NBs appeared apoptotic, with abnormally condensed DAPI staining.

(C) $12 \%(17 / 140)$ of traip ${ }^{-}$MB-NBs have irregular, ruffled nuclear envelope morphology.

(E) $11 \%(15 / 140)$ of traip ${ }^{-}$MB-NBs have micronuclei.

(E) 9\% (12/140) of traip MB-NBs have multiple nuclei.

(F) 9\% (13/140) of traip MB-NBs appeared polyploid, with abnormally large nuclei.

(G) Most MB-NBs from traip + OK107-GAL4 > GFP::Traip rescues have wild-type nuclear envelope morphology (96\%, 193/202), and 4\% (9/202) had ruffled nuclear envelope morphology.

(H) Control $3^{\text {rd }}$ instar larval CB-NB expressing His2Av::mRFP. Chromosomes segregate effectively during mitosis. $\mathrm{N}=10 \mathrm{NBs}$. See Movie 1.

(I) $26 \%$ (5/19) of traip CB-NBs form chromosome bridges during anaphase. A yellow arrowhead points to a chromosome as it pulls away in mid-anaphase, and a yellow arrow points to the chromosome bridge stretched across the constricting furrow. See Movie 2.

(J) Control 24 hours APF pupae expressing OK107-GAL4 > NLS::mCherry + mCD8::GFP show uniform fluorescence levels of both markers across the field of KCs, except for NBs (yellow outlines) and their immediate daughters which have reduced fluorescence.

(K) traip pupae contain patches of presumably aneuploid KCs with either double or no fluorescence for one or both markers (white outlines).

(L) Number of aneuploid KC clones per brain hemisphere in control, traip-, and traip + OK107-GAL4 > GFP::Traip rescue at 24 and 72 hours APF. Two-tailed t-test was used for significance. ns $=$ not significant, $* * * * \mathrm{p}<0.0001 . \mathrm{N} \geq 22$ hemispheres.

Scale bars $=5 \mu \mathrm{m}(\mathrm{A}-\mathrm{I}), 10 \mu \mathrm{m}(\mathrm{J}, \mathrm{K})$. 
bioRxiv preprint doi: https://doi.org/10.1101/2021.07.07.451466; this version posted July 7, 2021. The copyright holder for this preprint (which

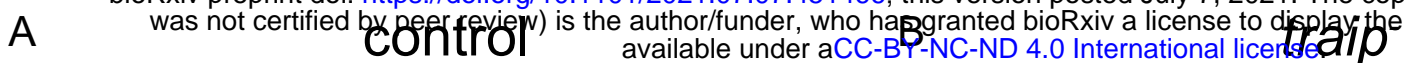
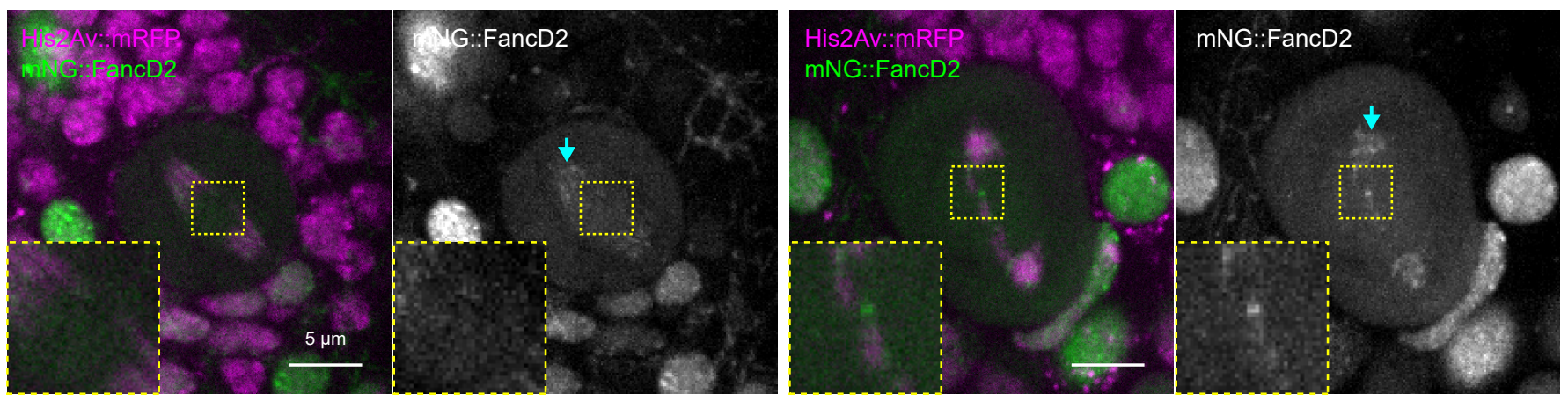

C

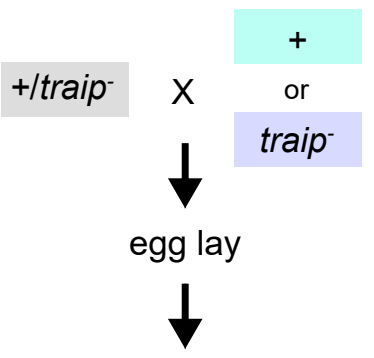

larval drug treatment

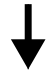

score adult eclosion

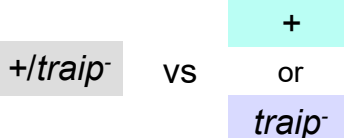

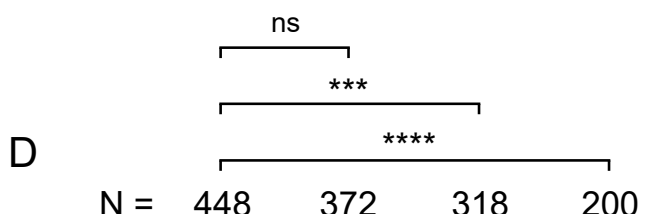

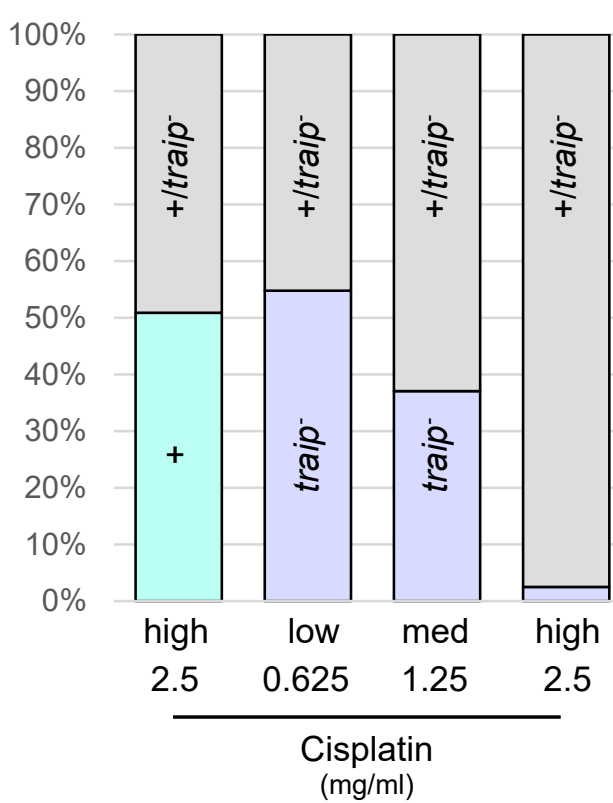

traip +

$E$

control

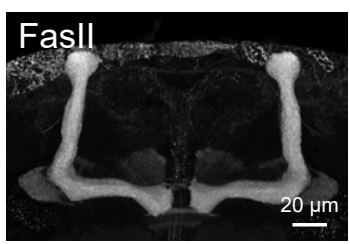

traip- +

G GFP ::Traip ${ }^{18 D, A 10 E}$

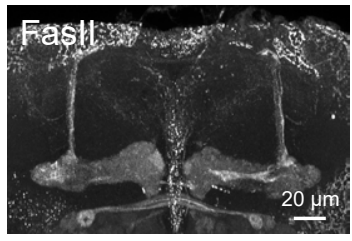

traip- +

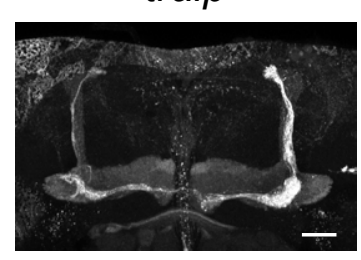

GFP::Traip ${ }^{L 12 E, G 14 D}$

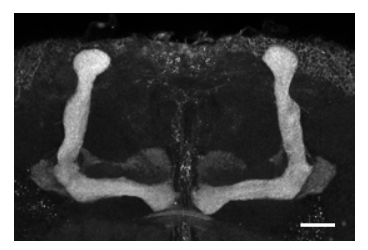

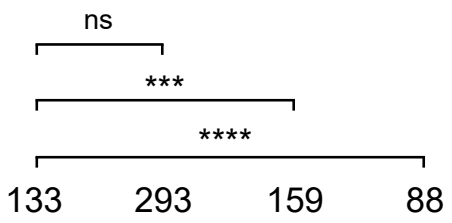
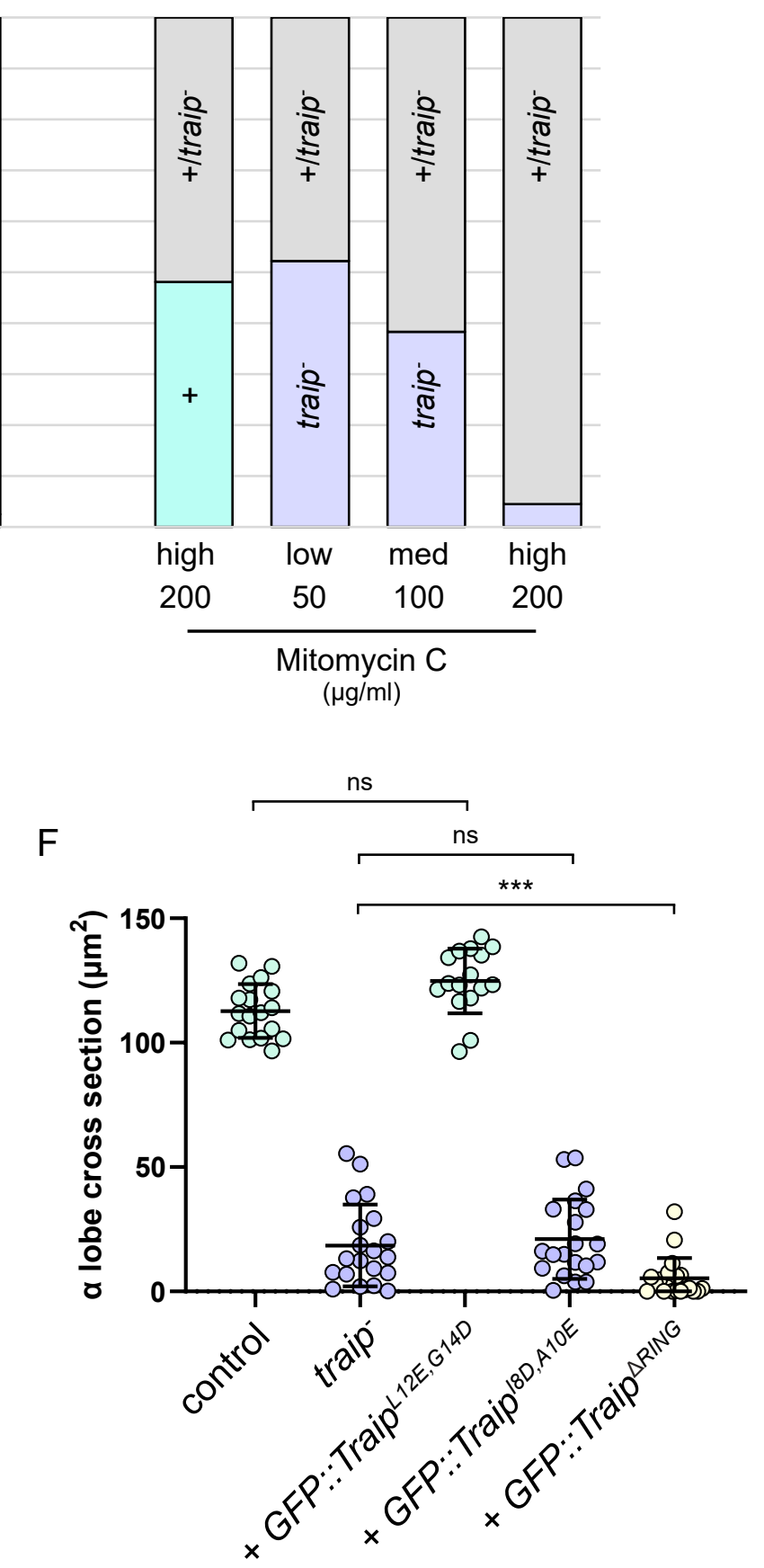

Figure 6 - Traip functions in inter-strand crosslink repair 


\section{Figure 6 - Traip is required for inter-strand crosslink repair}

(A, B) $3^{\text {rd }}$ instar larval CB-NBs expressing mNG::FancD2 (green, gray) with His2Av::mRFP (magenta). 7/12 traip NBs with mitotic DNA bridges had mNG::FancD2 puncta localized on the bridge (B, inset). Both controls and traip ${ }^{-}$have weak localization of mNG::FancD2 on mitotic chromosomes (cyan arrows). Scale bars $=5 \mu \mathrm{m}$.

(C) Experimental setup for drug treatment survival assays. $+/$ traip $^{-}$(traip ${ }^{\text {Exc142 }} /$ CyO ) females were mated to either + (cyan) or traip ${ }^{\Delta}$ (purple) males. The females laid eggs for 24 hours, which were aged for another 24 hours before adding drug. The larvae developed and adult eclosion was scored. Offspring were either $+/ \operatorname{traip}^{-}\left(+/\right.$traip $\left.^{\Delta}\right)$ vs $+(+/$ CyO $)$ for control crosses (cyan), or $+/ \operatorname{traip}^{-}\left(\operatorname{traip}^{\Delta} / \mathrm{CyO}\right)$ vs $\operatorname{traip}^{-}$ (traip $^{\Delta} /$ traip $^{\text {Exc142 }}$ ) for traip ${ }^{-}$crosses (purple).

(D) Drug treatment survival assay results. Control crosses produced roughly similar numbers of + vs + / traip offspring with high doses of inter-strand crosslinking agents Cisplatin or Mitomycin C. traip crosses also produced similar numbers of traip ${ }^{-} v+/$ traip $^{-}$offspring with low doses of either drug, but medium doses were semi-lethal and high doses were almost fully lethal to traip offspring. Chi-squared tests were used to determine significance. ns = not significant, $* * * p<0.001, * * * * p<0.0001$.

$(E, G, H) M B s$ from stained for Fasll. Genotypes are: control, traip traip $^{-}+$GFP::Traip ${ }^{L 12 E, G 14 D}(E) ;$ traip $^{-}+$ GFP::Traip ${ }^{I 8 D, A 1 O E}(\mathrm{G})$; traip ${ }^{-}+$GFP::Traip ${ }^{\triangle R I N G}(\mathrm{H})$. Transgene expression was driven by OK107-GAL4. Scale bars $=20 \mu \mathrm{m}$.

(F) $\alpha$ lobe cross-section measurements of control, traip', and traip ${ }^{-}+$GFP::Traip RING mutant variants. Two-tailed t-tests were used for significance. ${ }^{* * *} \mathrm{p}=0.0004 . \mathrm{N} \geq 16 \mathrm{MBs} /$ genotype. 


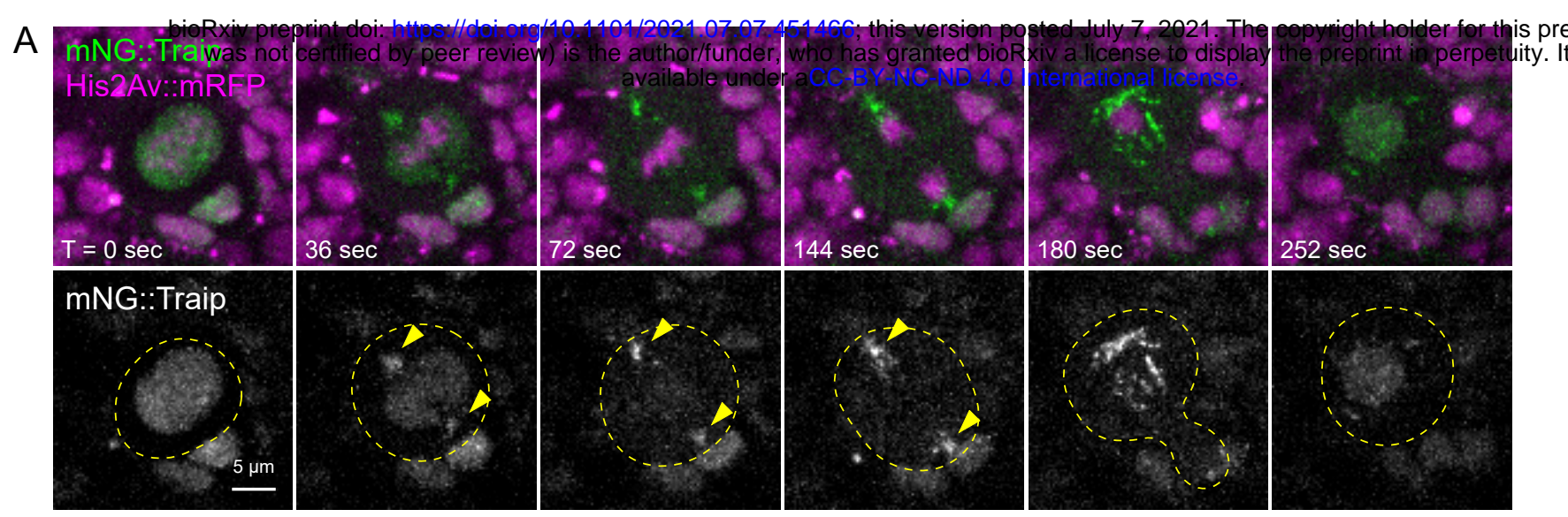

B

mNG::Traip mCherry: Tub

C

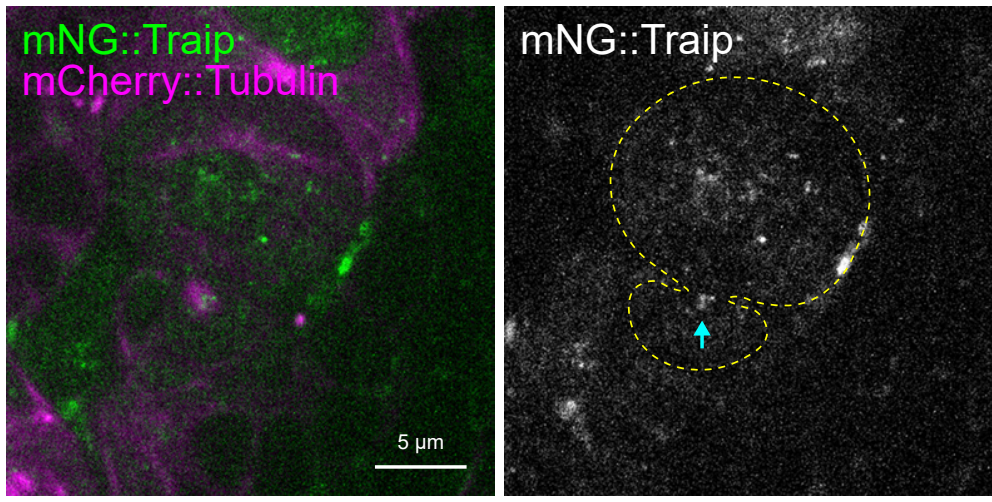

$\mathrm{H}$

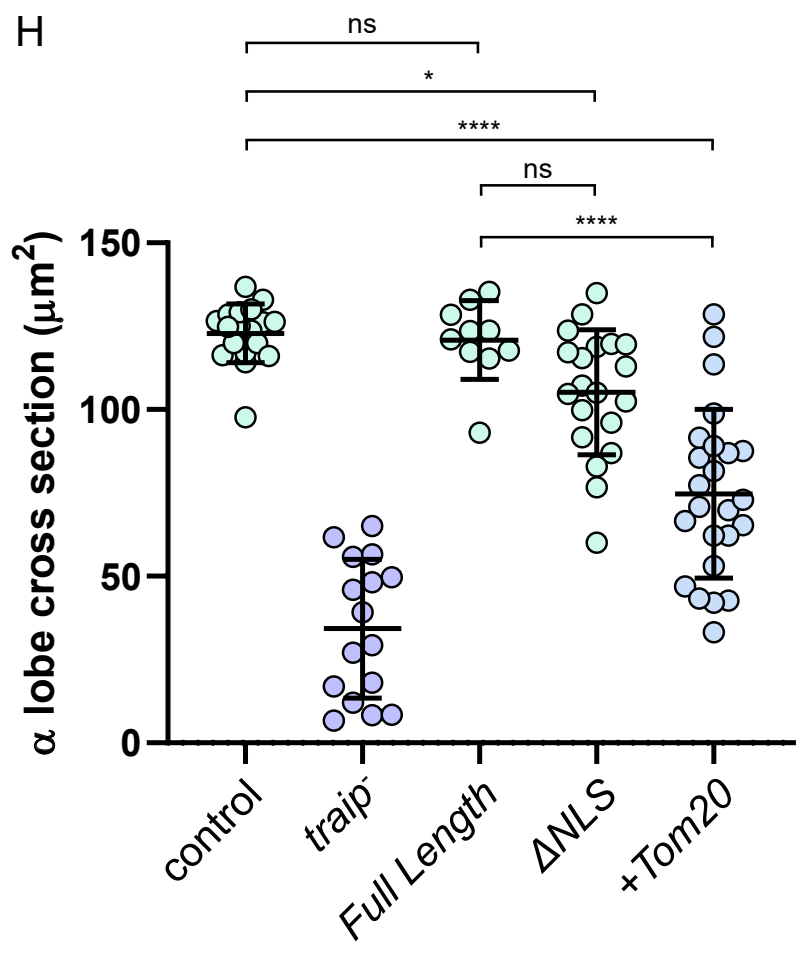

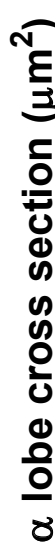

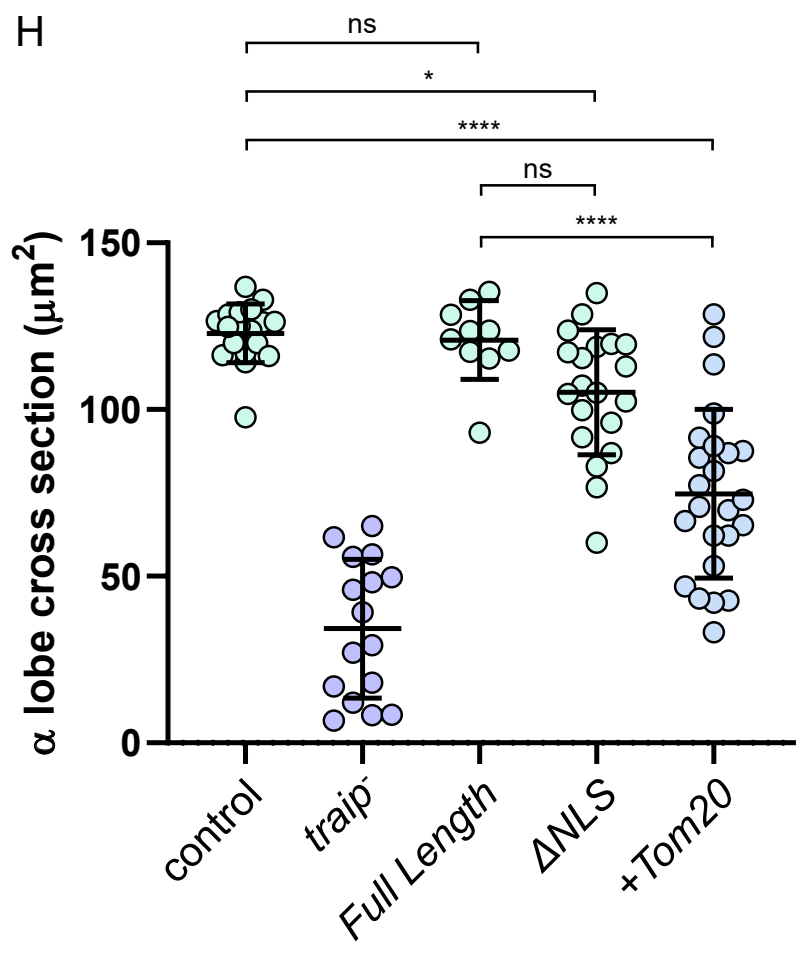

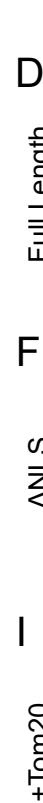

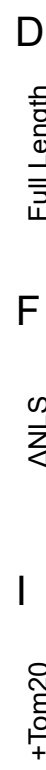
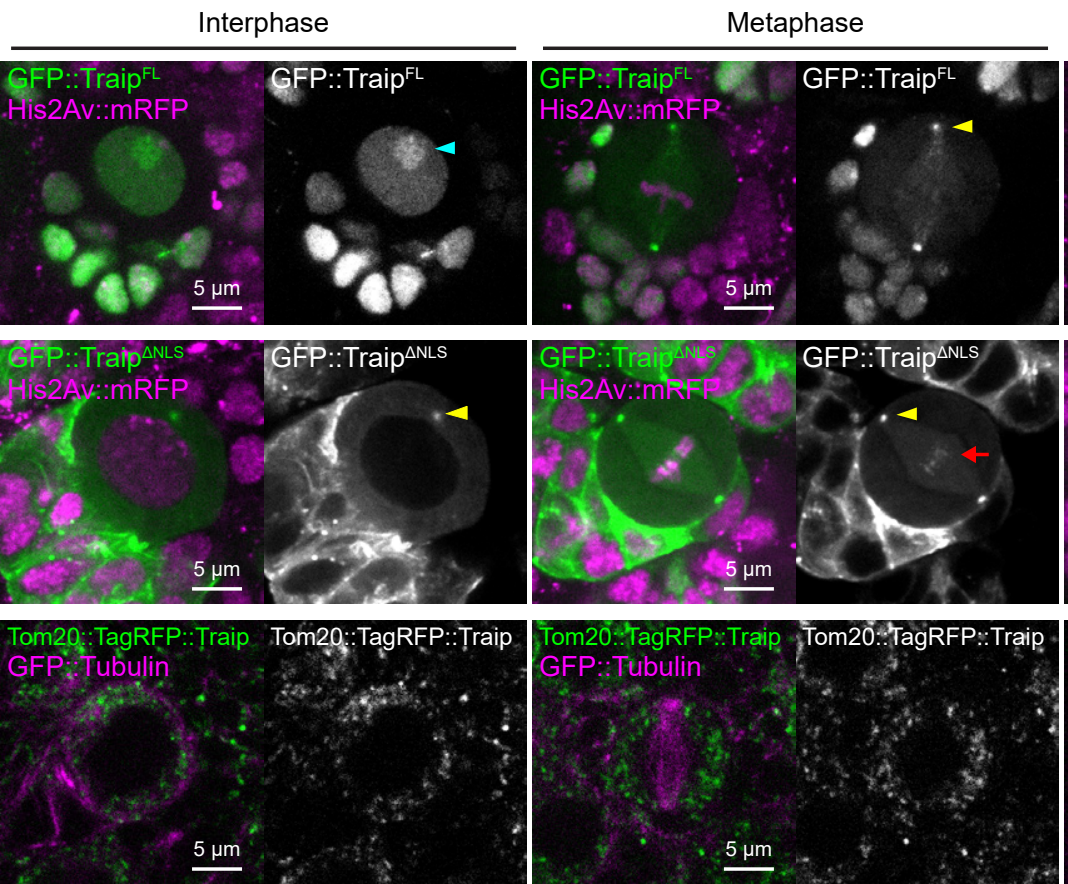

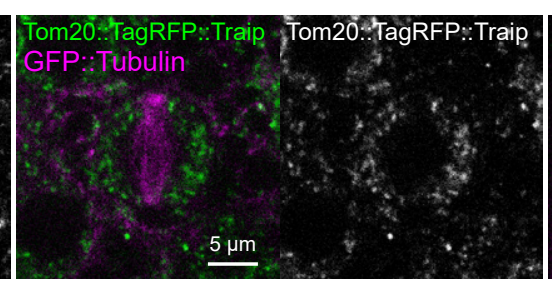

Figure 7 - Traip has dynamic localization in mitosis 


\section{Figure 7 - Traip has dynamic localization in mitosis}

(A) Live imaging of $\mathrm{mNG}:$ Traip (green, gray) with His2Av::mRFP (magenta) in $3^{\text {rd }}$ instar larval CB-NB mitosis. At mitotic onset ( $36 \mathrm{sec}$ ) mNG::Traip is released from the nucleus and localizes to the centrosomes (yellow arrowheads) and spindle. See Movie 3.

(B-C) High magnification live imaging of $\mathrm{mNG}:$ :Traip (green, gray) with wor-GAL4 > mCherry::Tubulin (magenta). mNG::Traip forms puncta that travel poleward on the spindle and coalesce at the centrosomes (yellow arrowheads, B), and coalesces at the cytokinetic furrow and midbody in late mitosis (cyan arrow, C). See Movie 4.

(D, F) Localization of GFP::Traip variants (green) expressed via wor-GAL4 with His2Av::mRFP (magenta) in CB-NBs during interphase, metaphase, and late mitosis. (D) GFP::Traip ${ }^{\text {Full Length }}{ }^{\text {has nucleolar }}$ localization in interphase (cyan arrowhead), centrosome localization in mitosis (yellow arrowhead), and furrow localization in late mitosis (cyan arrow). (F) GFP::Traip ${ }^{\triangle N L S}$ lacks nuclear localization in interphase, but has centrosome localization in interphase and mitosis (yellow arrowhead), furrow localization in late mitosis (cyan arrow), and also localizes to metaphase chromosomes (red arrow).

$(E, G, J) M B s$ stained for Fasll. Genotypes are: $\operatorname{traip}^{-}+G F P:: \operatorname{Traip}^{F L}(\mathrm{E}) ; \operatorname{traip}^{-}+G F P:: \operatorname{Traip}^{\triangle N L S}(\mathrm{G})$; and traip + Tom20::TagRFP::Traip (J). Transgene expression was driven by OK107-GAL4.

(H) $\alpha$ lobe cross-section measurements of control, traip ${ }^{-}$traip $^{-}+$GFP::Traip ${ }^{F L}$, traip $^{-}+$GFP::Traip ${ }^{\Delta N L S}$, and traip + Tom20::TagRFP::Traip. Ordinary one-way ANOVA with Tukey's test was used for significance. ns $=$ not significant, $* \mathrm{p}=0.0448, * * * * \mathrm{p}<0.0001 . \mathrm{N} \geq 16 \mathrm{MBs}$.

(I) Localization of Tom20::TagRFP::Traip (green) with GFP::Tubulin (magenta) in CB-NBs. Tom20::TagRFP fails to localize to the nucleus in interphase, and is absent from the centrosome and spindle region in mitosis.

Scale bars $=5 \mu \mathrm{m}(A-C, D, F, I), 20 \mu m(E, G, J)$. 
bioRxiv preprint doi: https://doi.org/10.1101/2021.07.07.451466; this version posted July 7, 2021. The copyright holder for this preprint (which was not certified by peer review) is the author/funder, who has granted bioRxiv a license to display the preprint in perpetuity. It is made

A available under aCC-BY-NC-ND 4.0 International license.

\section{Inter-strand crosslink \\ Attached sister chromatids \\ Resolution via Traip \\ Proper}

$\frac{0}{2}$
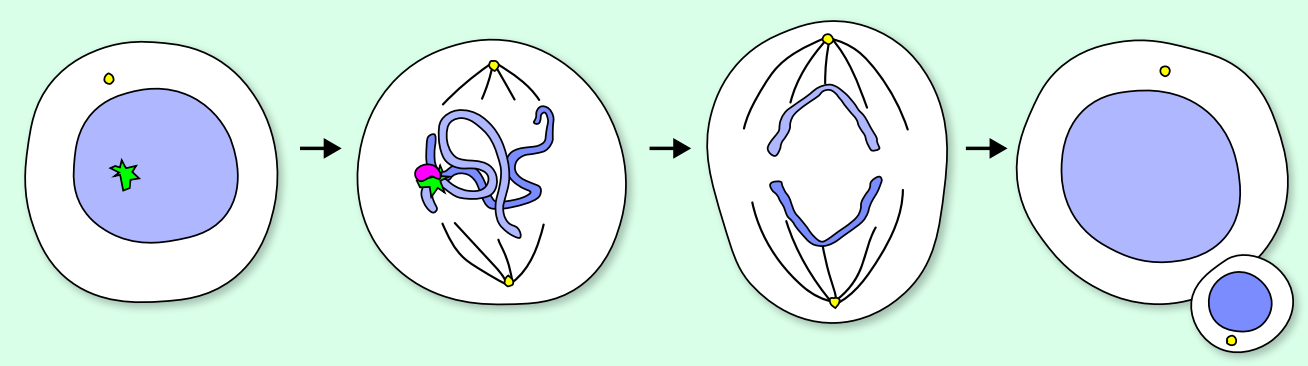

等 $\begin{aligned} & \text { Inter-strand } \\ & \text { crosslink }\end{aligned}$

Traip

Centrosome
B

Cytokinesis Polyploidy or blocked multinucleate

$\frac{2}{0}$
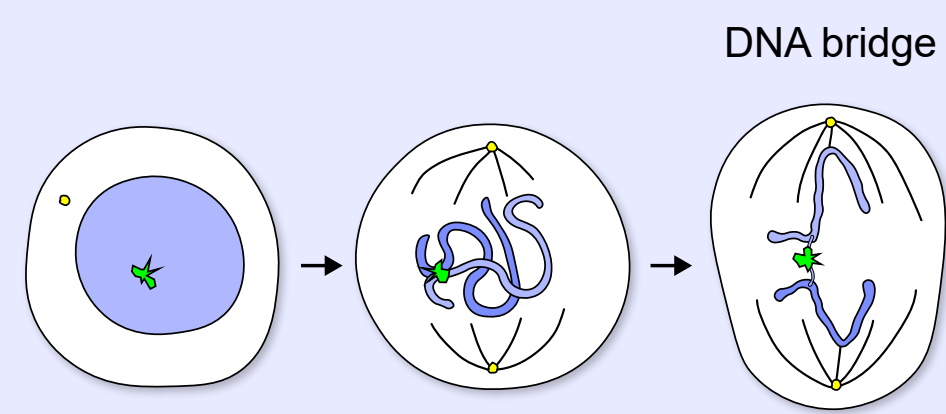
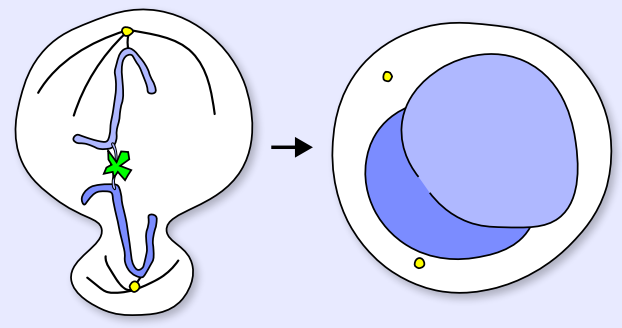

$\forall$

Cell death and reduced neurogenesis

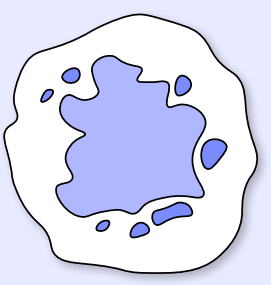


bioRxiv preprint doi: https://doi.org/10.1101/2021.07.07.451466; this version posted July 7, 2021. The copyright holder for this preprint (which

was not certified by peer review) is the author/funder, who has granted bioRxiv a license to display the preprint in perpetuity. It is made available under aCC-BY-NC-ND 4.0 International license.

\section{Figure 8 - Model of Traip function in neurogenesis}

(A) In wild type, inter-strand crosslinks may prevent completion of DNA replication, resulting in sister chromatids remaining partially attached in mitosis. Traip initiates the resolution of these attached sister chromatids, thereby ensuring chromosome segregation and promoting proper neurogenesis.

(B) In traip", attached sister chromatids are not properly resolved, leading to DNA bridges in mitosis. DNA bridges could block cytokinesis, leading to formation of polyploid or multinucleated cells (top path). Alternatively, DNA bridges could break during mitosis, leading to formation of aneuploid cells or micronuclei (bottom path). These defects could result in cell cycle exit, apoptosis, and ultimately reduced neurogenesis. 


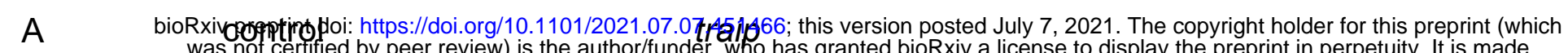
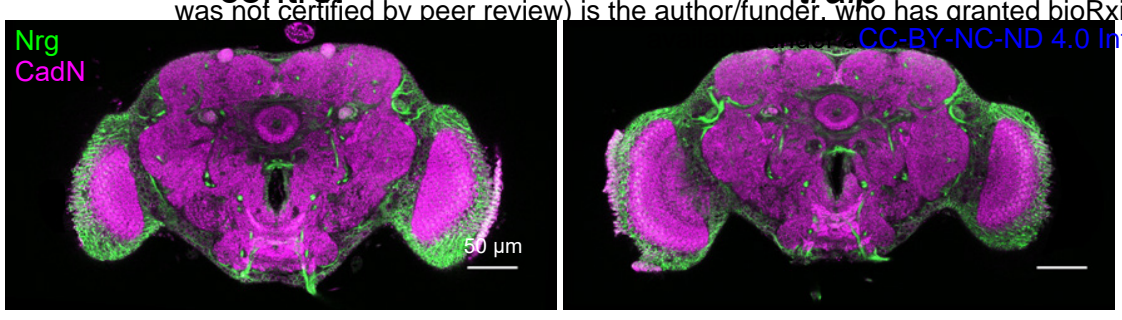

$\mathrm{B}$
$\overline{0}$
은
음
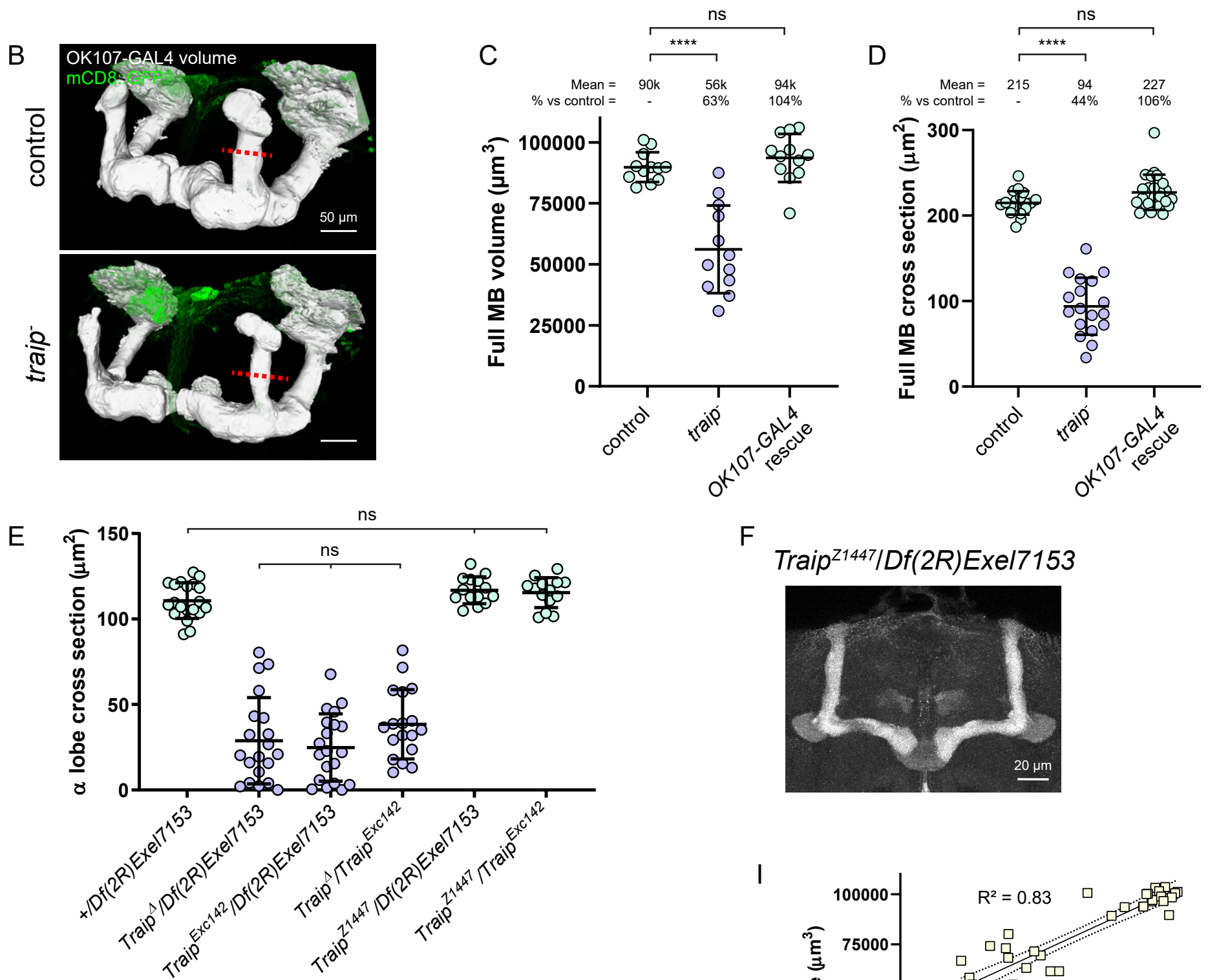

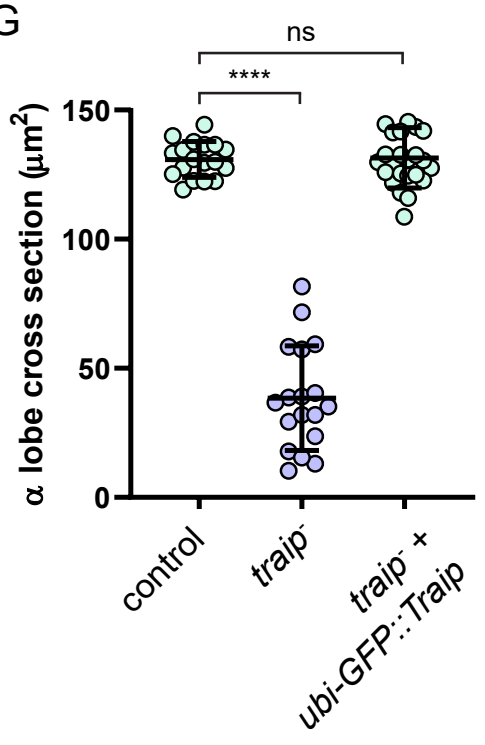

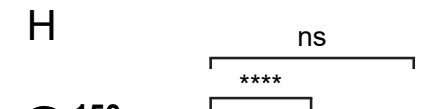

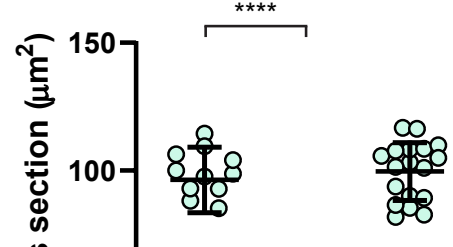

Figure S1 - Traip is required for proper MB structure, supporting figures 


\section{Figure S1 - Traip is required for proper MB structure, supporting figures}

(A) Confocal slices through control and traip- adult brains stained for $\mathrm{N}$-Cadherin (CadN, magenta) and Neuroglian ( $\mathrm{Nrg}$, green), showing no obvious differences in neuropil regions or axon tracts, excluding the MBs.

(B) Control and traip full MB volumes (white) segmented from OK107-GAL4 > mCD8::GFP fluorescence (green).

(C) Full MB volume measurements. $\mathrm{N} \geq 18 \mathrm{MBs}$.

(D) $\alpha^{\prime} / \alpha$ lobe cross-section measurements. $N \geq 18$ MBs.

(E) $\alpha$ lobe cross-section measurements of controls and various combinations of Traip mutant alleles. Homozygous null animal MBs are similarly reduced, whereas hypomorphic animals have wild-type MB size. $\mathrm{N} \geq 18 \mathrm{MBs} /$ genotype.

(F) Hypomorphic traip ${ }^{21447}$ MBs have wild-type morphology.

$(\mathrm{G}, \mathrm{H}) \propto$ lobe cross-section measurements show that traip ${ }^{-}+$ubi-GFP::Traip $(\mathrm{G})$ and traip ${ }^{-}+$Tub-GAL4 > $^{-}$ GFP::Traip $(\mathrm{H})$ rescues have wild-type MB size. $\mathrm{N} \geq 10 \mathrm{MBs}$.

(I). Simple linear regression between KC number ( $\mathrm{X}$ axis) and volumes ( $\mathrm{Y}$ axis) of OK107-GAL4 > mCD8::GFP-positive full MBs ( $R^{2}=0.83$, yellow squares) or Fasll-positive $\alpha / \beta$ lobes $\left(R^{2}=0.84\right.$, blue circles).

(J) Simple linear regression between KC number ( $\mathrm{X}$ axis) and the cross sectional areas ( $\mathrm{Y}$ axis) of OK107GAL4 > mCD8::GFP-positive $\alpha^{\prime} / \alpha$ lobes $\left(R^{2}=0.87\right.$, yellow squares $)$ or Fasll-positive $\alpha$ lobes $\left(R^{2}=0.87\right.$, blue circles).

Two-tailed Mann-Whitney test was used for significance. $\mathrm{ns}=$ not significant, $* * * * p<0.0001$. Scale bars $=50 \mu \mathrm{m}(\mathrm{A}, \mathrm{B}), 20 \mu \mathrm{m}(\mathrm{F})$. 


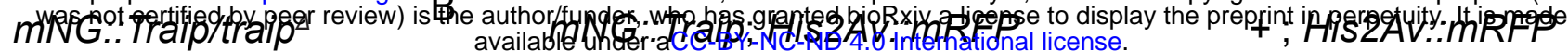
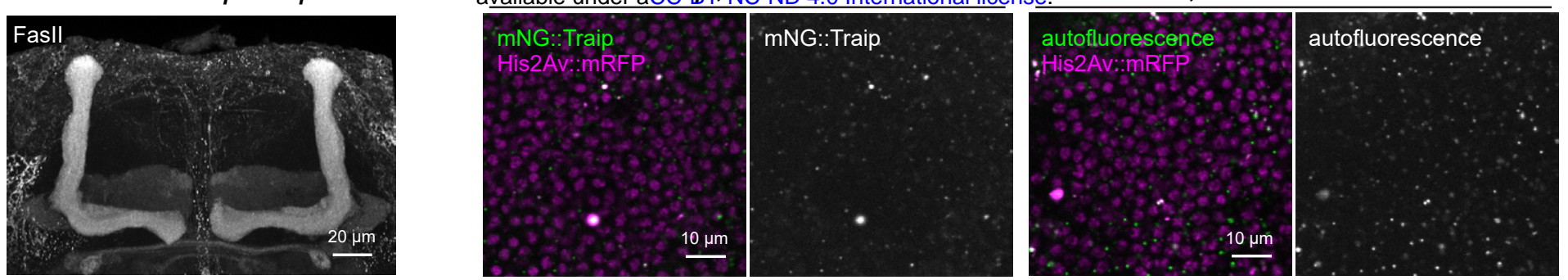

C

traip- + wor-GAL4> GFP::Traip

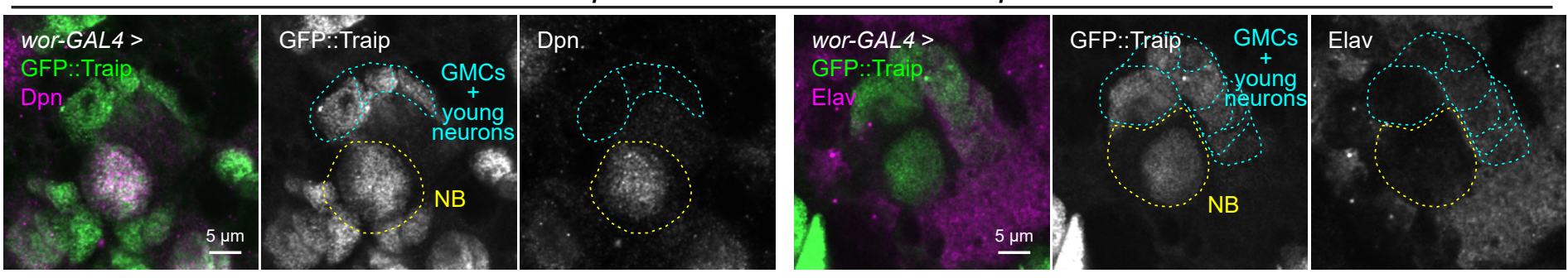

D

traip- + elav-GAL4 > GFP::Traip

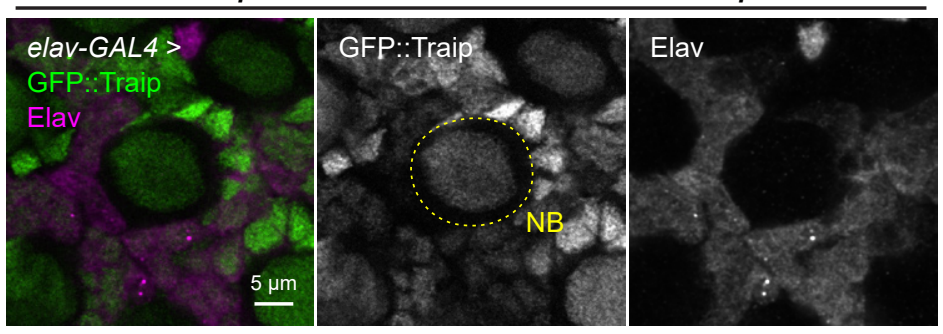

E $\frac{\text { traip }^{-}+n S y b-G A L 4>\text { GFP:: Traip }}{\text { Larval brain Adult brain }}$

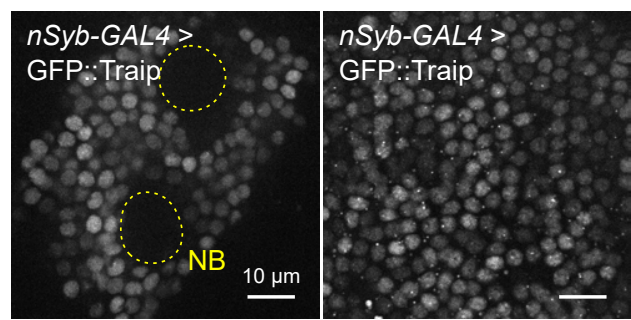




\section{Figure S2 - Traip is required in neuroblasts, supporting figures}

(A) $m N G:: T_{\text {Traip }}^{\text {CRISPR }}$ encodes a functional protein that fully rescues traip ${ }^{\Delta} \mathrm{MB}$ size.

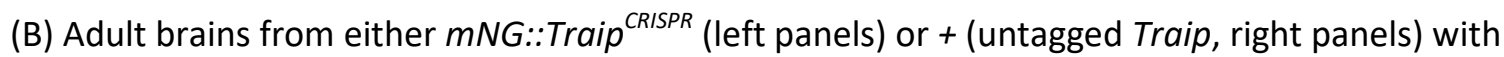
His2Av::mRFP (magenta). mNG::Traip (green, gray; left panels) does not have fluorescent signal above autofluorescence background of + (green, gray; right panels).

(C) CB-NBs from traip ${ }^{-}+$wor-GAL4 > GFP::Traip $3^{\text {rd }}$ instar larval brains, stained either Dpn (magenta; left panels) or Elav (magenta; right panels). wor-GAL4 > GFP::Traip (green) is expressed in NBs (Dpn-positive, yellow highlighting) and persists into daughter GMCs and neurons (Elav-positive, cyan highlighting).

(D) traip + elav-GAL4 > GFP::Traip has significant GFP::Traip expression in $3^{\text {rd }}$ instar larval CB-NBs.

(E) traip $+n S y b-G A L 4>$ GFP::Traip has GFP::Traip expression in larval and adult neurons, but no expression in larval NBs.

Scale bars $=10 \mu \mathrm{m}(\mathrm{B}, \mathrm{E}), 5 \mu \mathrm{m}(\mathrm{C}, \mathrm{D})$. 
bioRxiv preprint doi: https://doi.org/10.1101/2021.07.07.451466; this version posted July 7, 2021. The copyright holder for this preprint (which

A

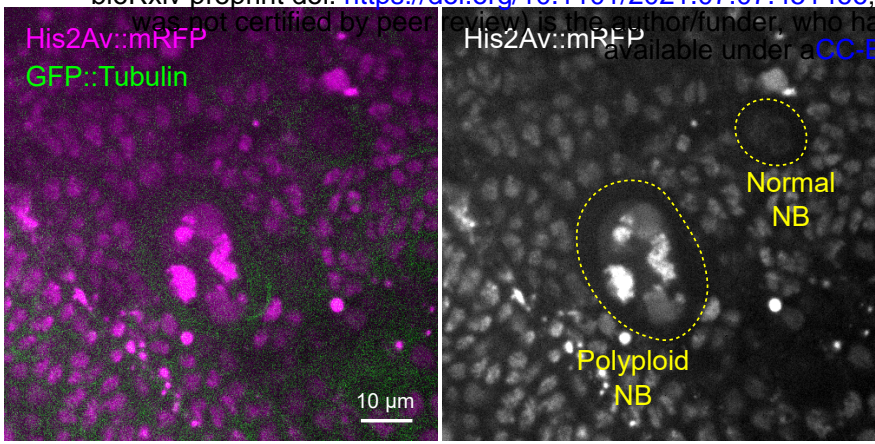
s granted bioRxiB license to display the preprint irf perpetuity. It is made 3Y-NC-ND 4.0 International license.

C

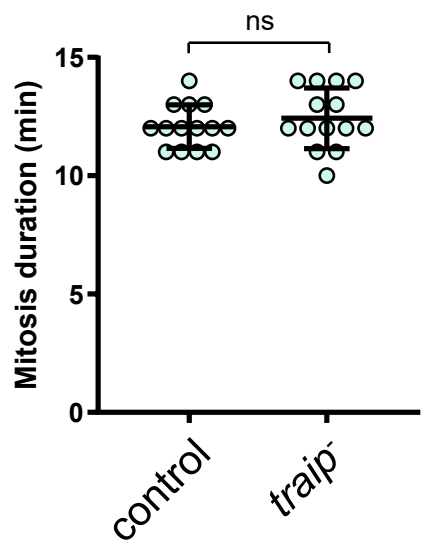

$\begin{array}{rcccccc}\text { Mean }= & - & 15 & 44 & - & 37 & 8 \\ \mathrm{~N}= & 0 & 31 & 3 & 0 & 51 & 1\end{array}$
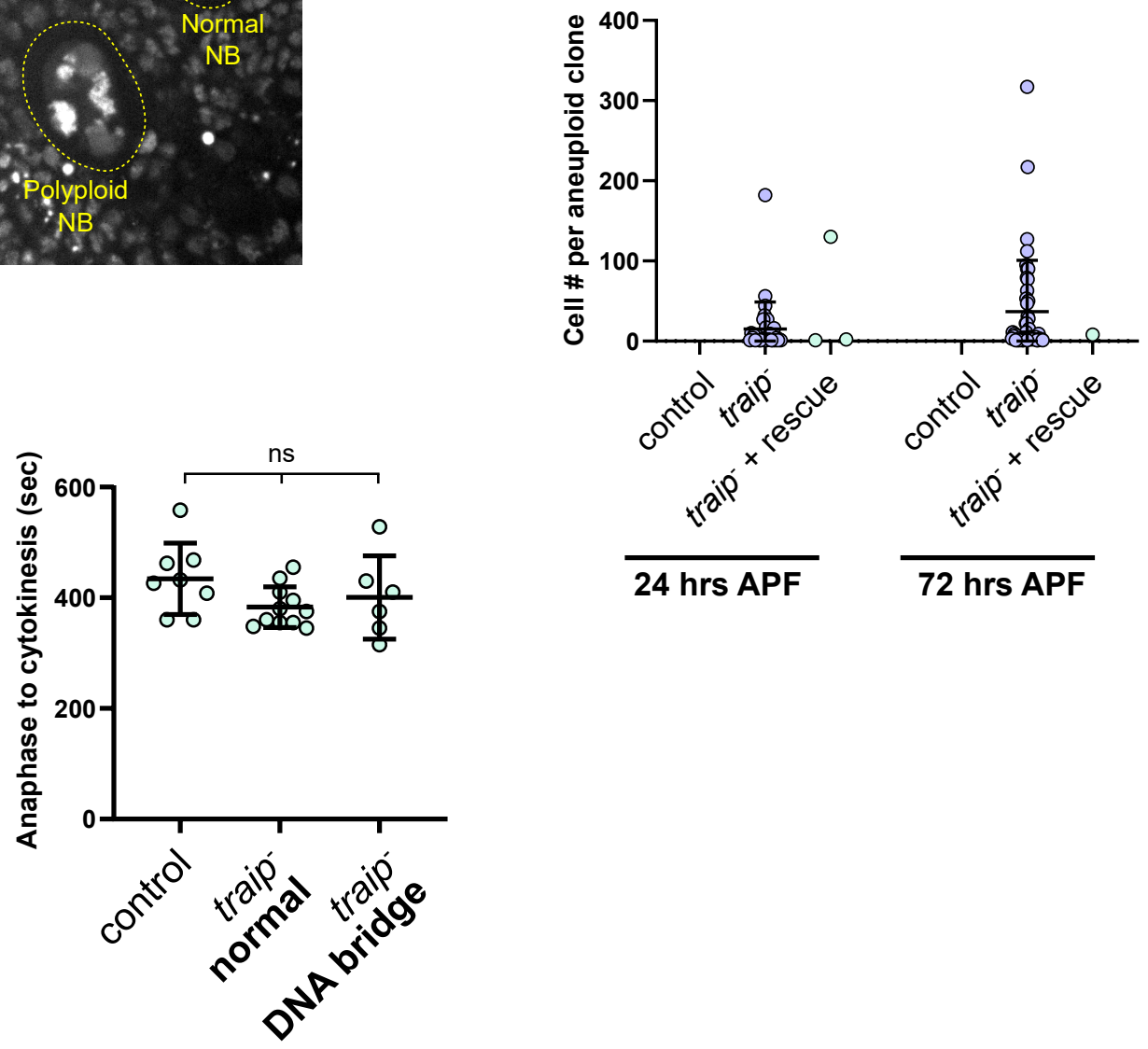

E $\quad 3 r d$ instar larval CB-NBs

$\mathrm{F}$

$24 \mathrm{hrs}$ APF MB-NBs
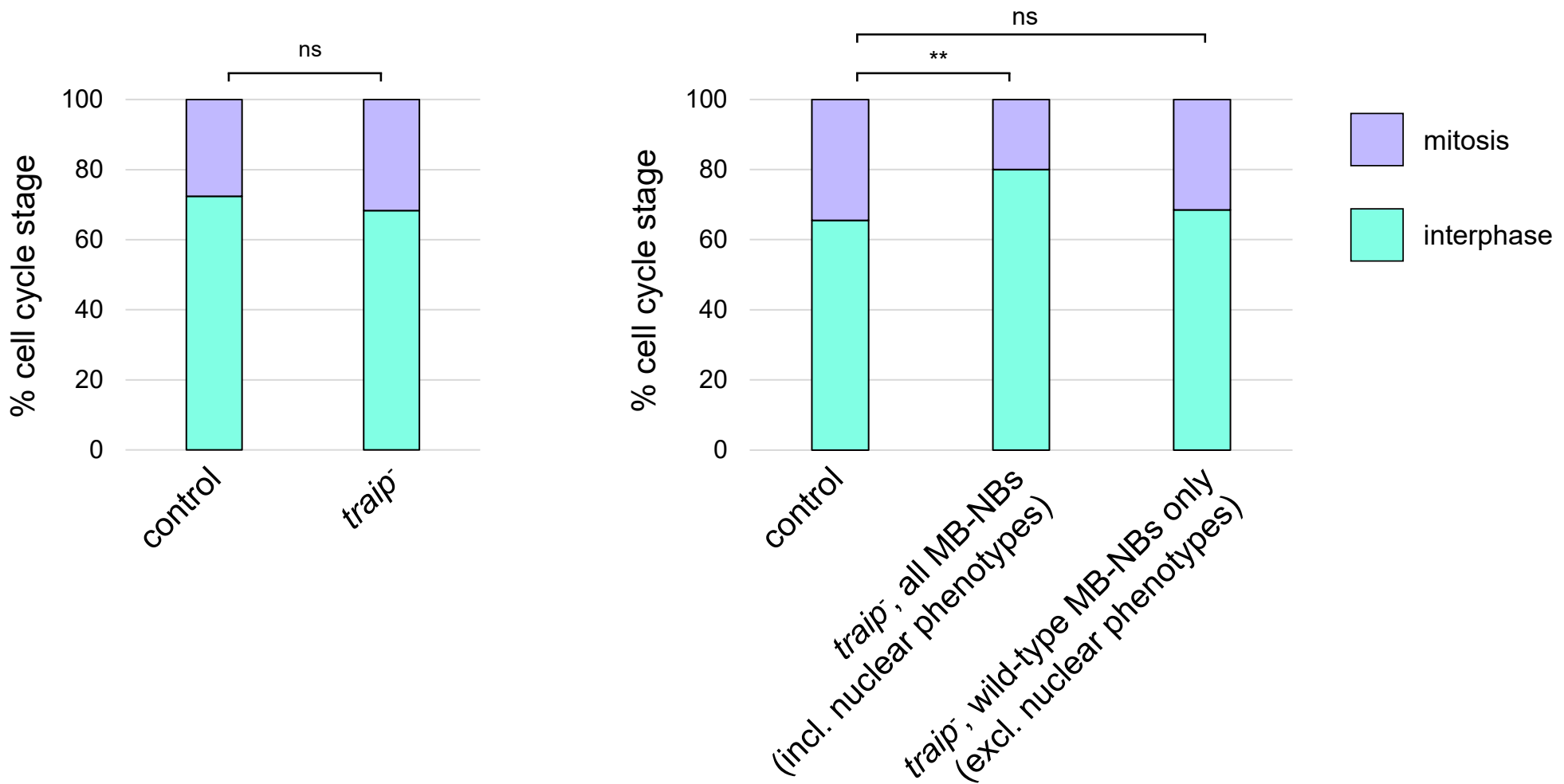

Figure S3 - Traip suppresses nuclear phenotypes and mitotic DNA bridges, supporting figures 


\section{Figure S3 - Traip suppresses multinuclear phenotypes and mitotic DNA bridges, supporting figures}

(A) Live imaging of traip $3^{\text {rd }}$ instar larval CB-NBs expressing His2Av::mRFP (magenta) and GFP::Tubulin (green) showing likely polyploid NBs with enlarged nuclei and increased His2Av::mRFP fluorescence compared to a normal NB. Scale bars $=10 \mu \mathrm{m}$.

(B) KC number per aneuploid clone in control, traip", and traip ${ }^{-}+$GFP::Traip rescue expressing OK107GAL4 > NLS::mCherry + mCD8::GFP at 24 and 72 hours APF pupal brains. The average number of KCs per clone in traip increases from 15 at 24 hours APF to 37 at 72 hours APF. * $p=0.0287$.

(C) Total duration of mitosis for control and traip $3^{\text {rd }}$ instar larval CB-NBs, as measured from prophase onset to full furrow constriction. $\mathrm{N}=14 \mathrm{NBs}$.

(D) Time from anaphase onset to full furrow constriction for control CB-NBs and traip ${ }^{-}$CB-NBs either with or without DNA bridges.

(E) Mitotic index of fixed control and traip $3^{\text {rd }}$ instar larval CB-NBs.

(F) Mitotic index of fixed control and traip 24 hours APF MB-NBs, either including all traip ${ }^{-}$MB-NBs or only traip ${ }^{-M B}-N B s$ without nuclear phenotypes (right column). ${ }^{* *} p=0.0025$.

Two-tailed Mann-Whitney test $(B)$, t-test $(C, D)$, or chi-squared test $(E, F)$ were used for significance. ns = not significant. 


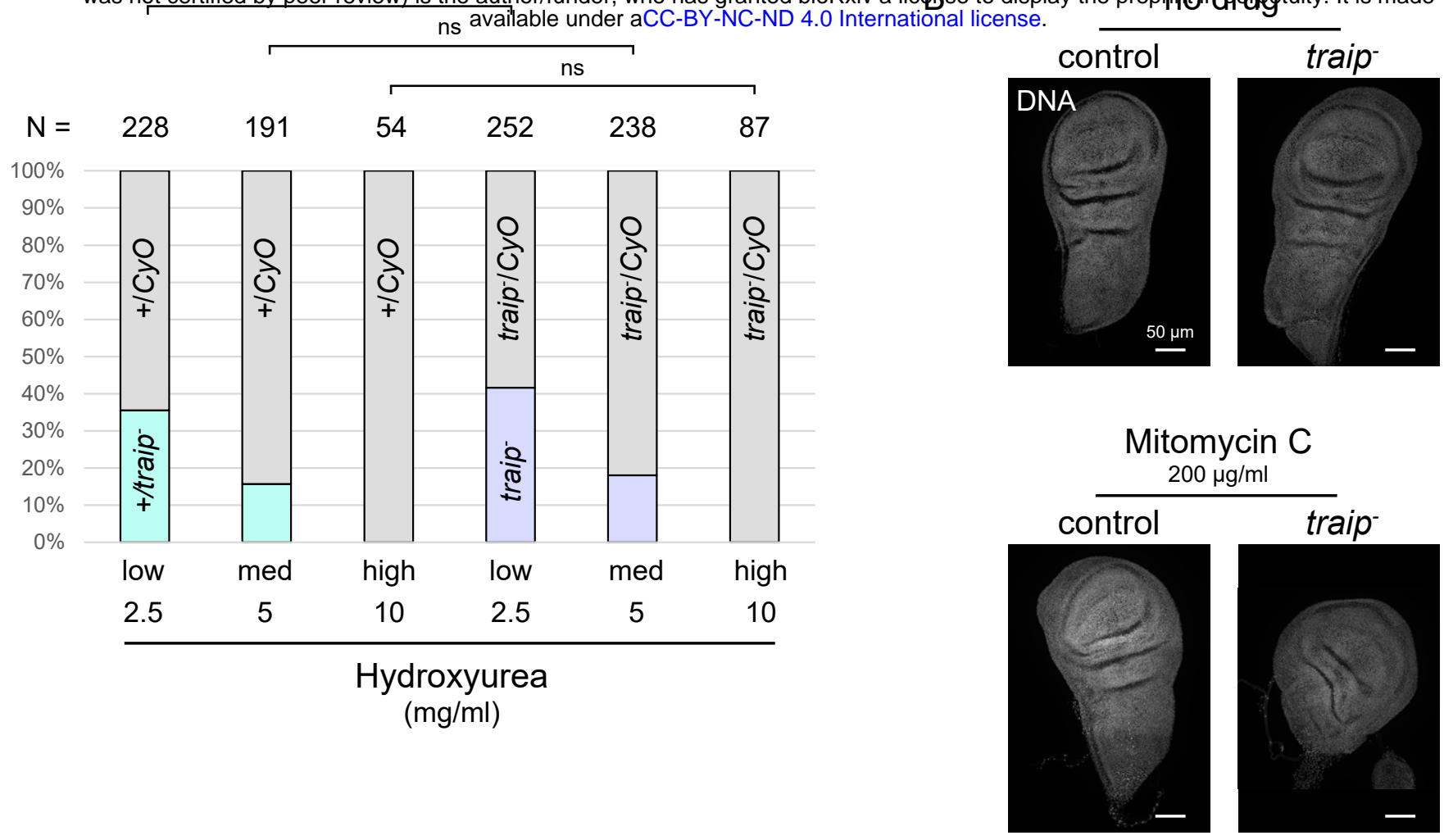

C

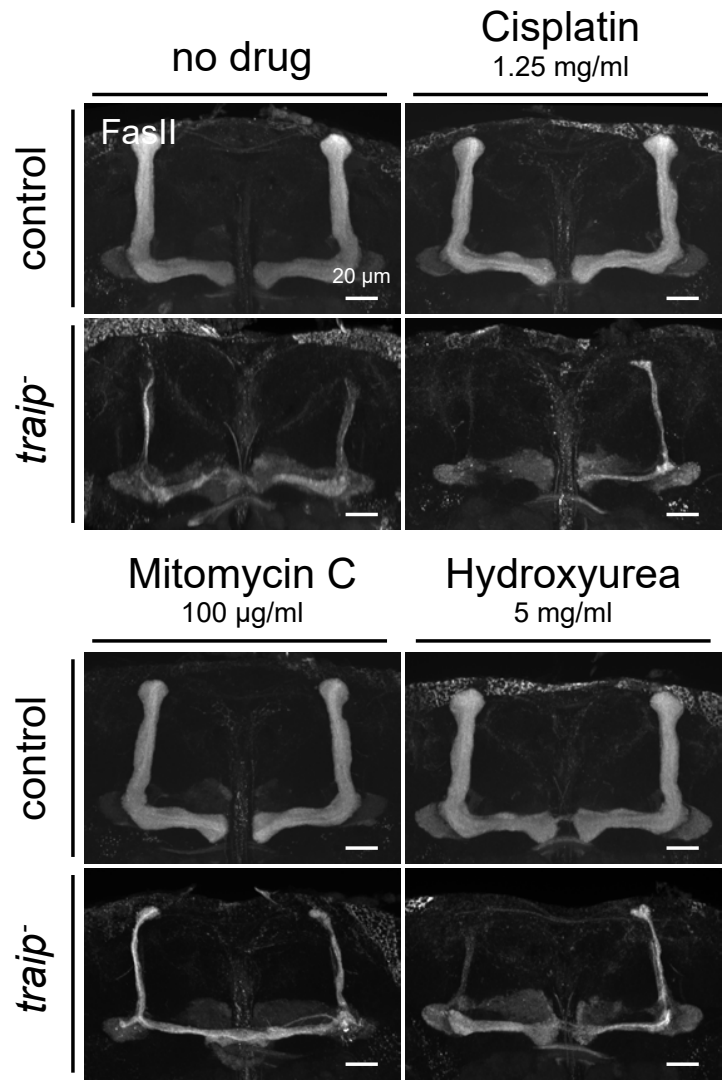

D

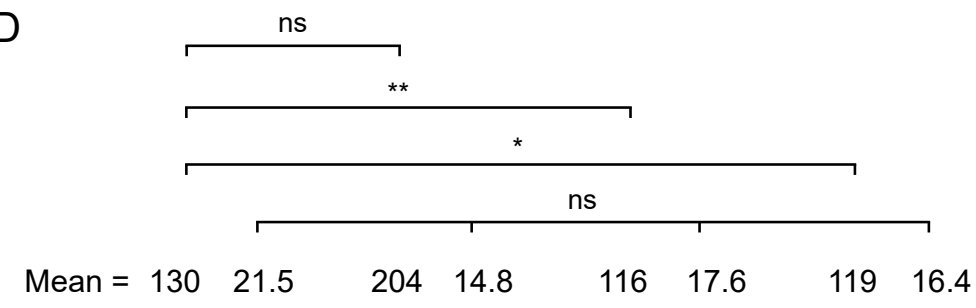

톨
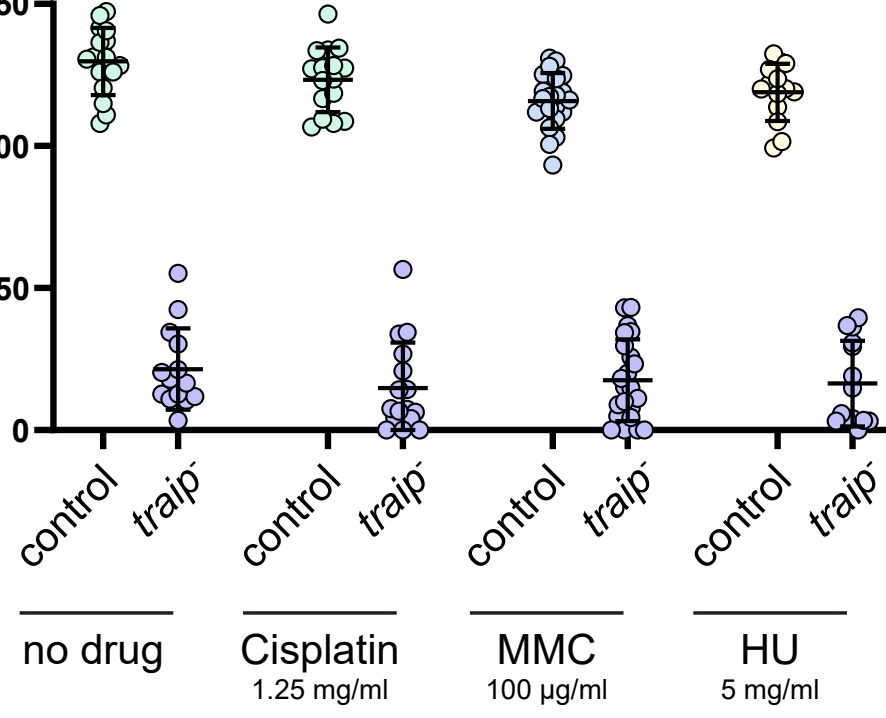


\section{Figure S4 - Traip is required for inter-strand crosslink repair, supporting figures}

(A) Hydroxyurea treatment survival assay results. Both control and traip crosses produced offspring that were increasingly sensitive to higher doses of hydroxyurea. Chi-squared tests were used for significance.

(B) Wing discs from control and traip $3^{\text {rd }}$ instar larvae treated with either no drug or high doses of Mitomycin C and stained for DAPI (gray). traip discs treated with Mitomycin C are severely reduced in size compared to controls.

(C) MBs from control and traip treated with no drug or medium doses of Cisplatin, Mitomycin C, or Hydroxyurea, stained with Fasll. Scale $=20 \mu \mathrm{m}$.

(D) $\alpha$ lobe cross-section measurements of control and traip ${ }^{-}$treated with no drug or medium doses of Cisplatin, Mitomycin C (MMC), or Hydroxyurea (HU). Ordinary one-way ANOVA with Tukey's test was used for significance. $n s=$ not significant, $* * p=0.0013, * p=0.0357 . N \geq 14$ MBs. 
bioRxiv preprint doi: https://doi.org/10.1101/2021.07.07.451466; this version posted July 7, 2021. The copyright holder for this preprint (which

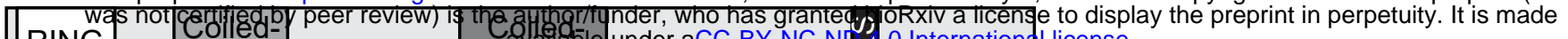

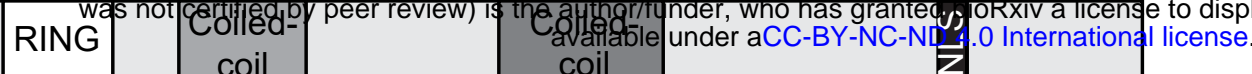

173-268

centrosome/spindle

\section{9-435}

centrosome/spindle

furrow

nucleolus

B

Interphase

Metaphase

Late Anaphase/Telophase

C

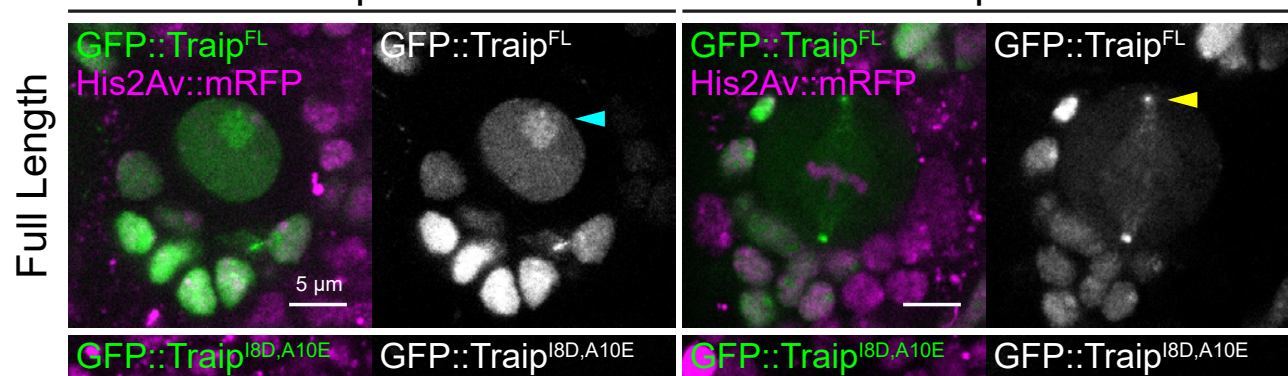

D

뚱

E

$$
\frac{N}{\frac{N}{}}
$$

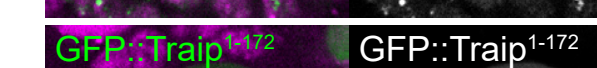

$\mathrm{F}$

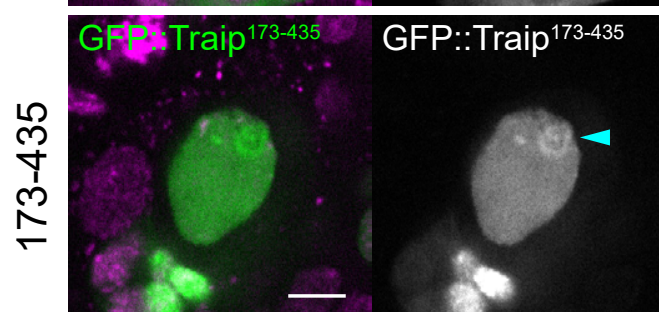

$\infty$
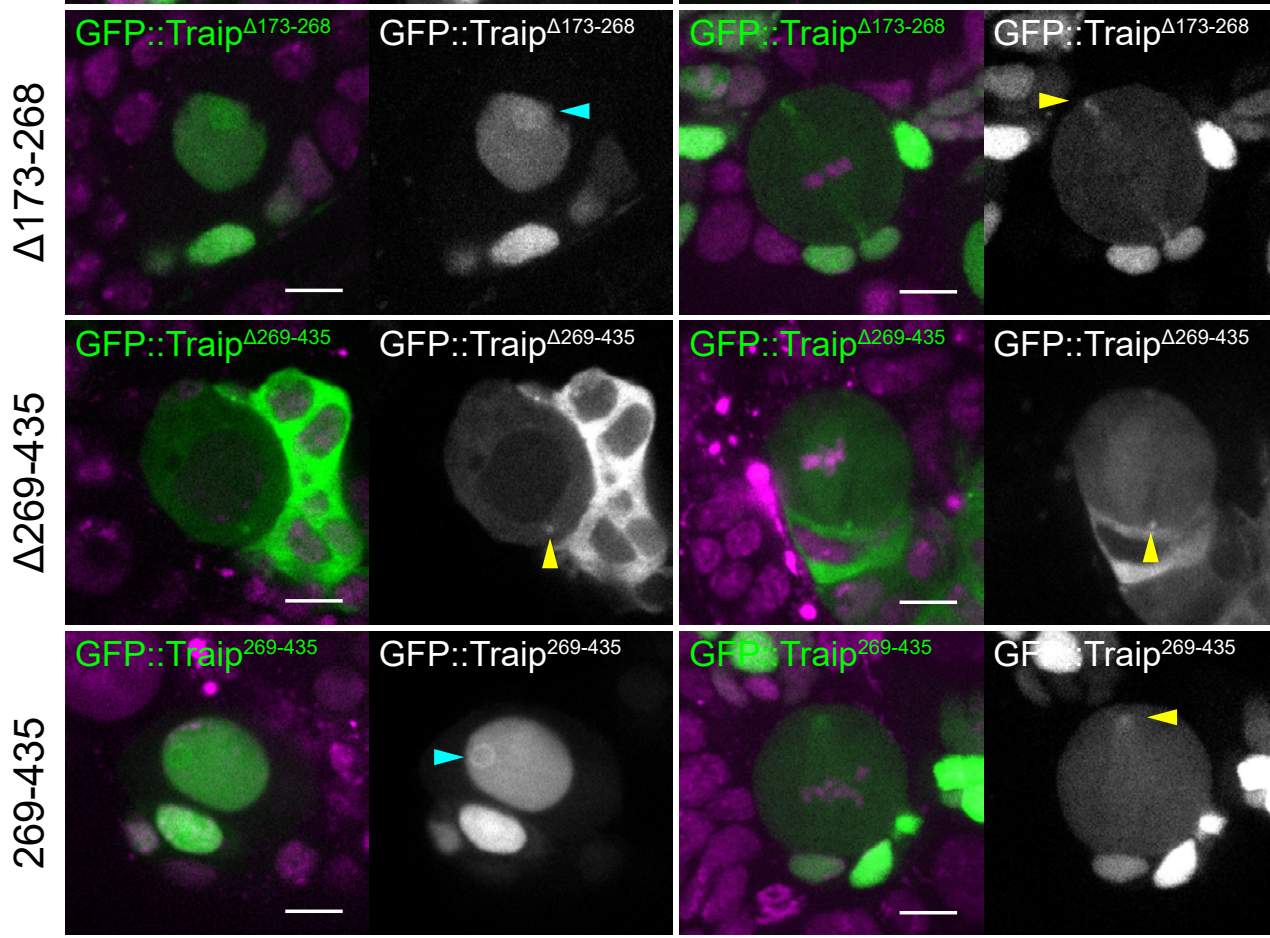

G
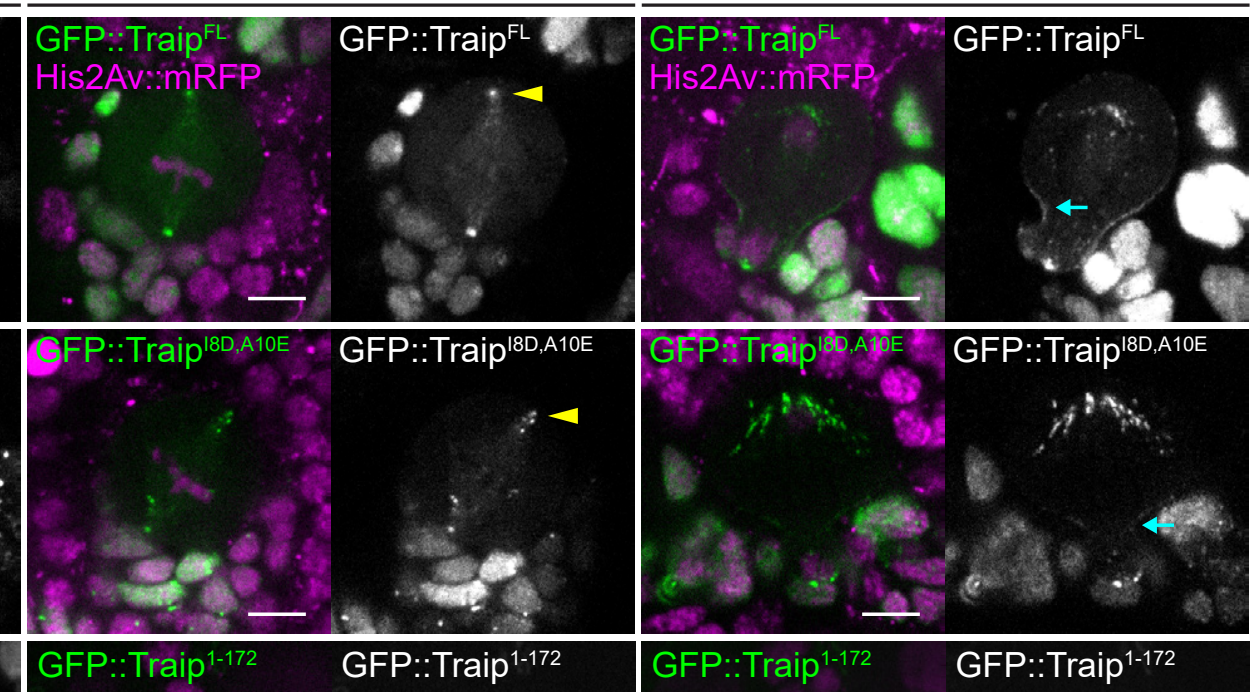

GFP:: Traip ${ }^{1}$

GFP::Traip ${ }^{1-172}$

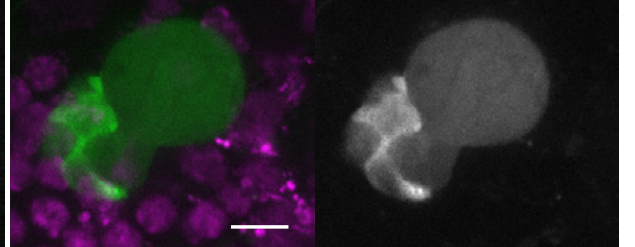

(1)

0

$\stackrel{2}{n}$

$\stackrel{\frac{m}{4}}{\frac{\pi}{4}}$

$\stackrel{\frac{m}{4}}{\frac{\pi}{4}}$

$\frac{\mathfrak{N}}{\stackrel{N}{T}}$

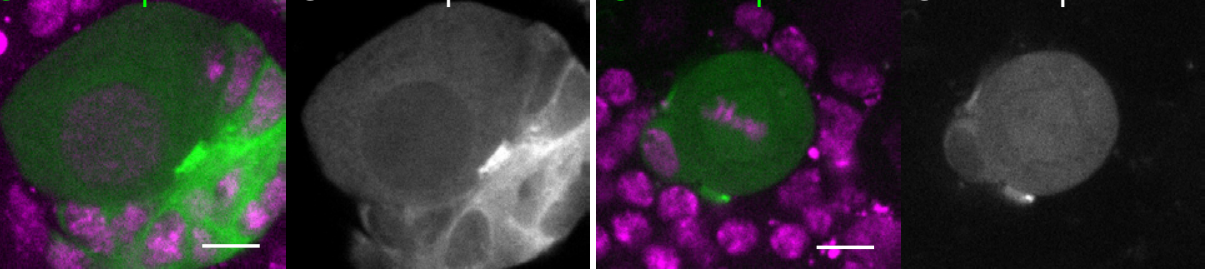

GFP::Traip $p^{173-485}$ GFP::Traip ${ }^{173-45}$
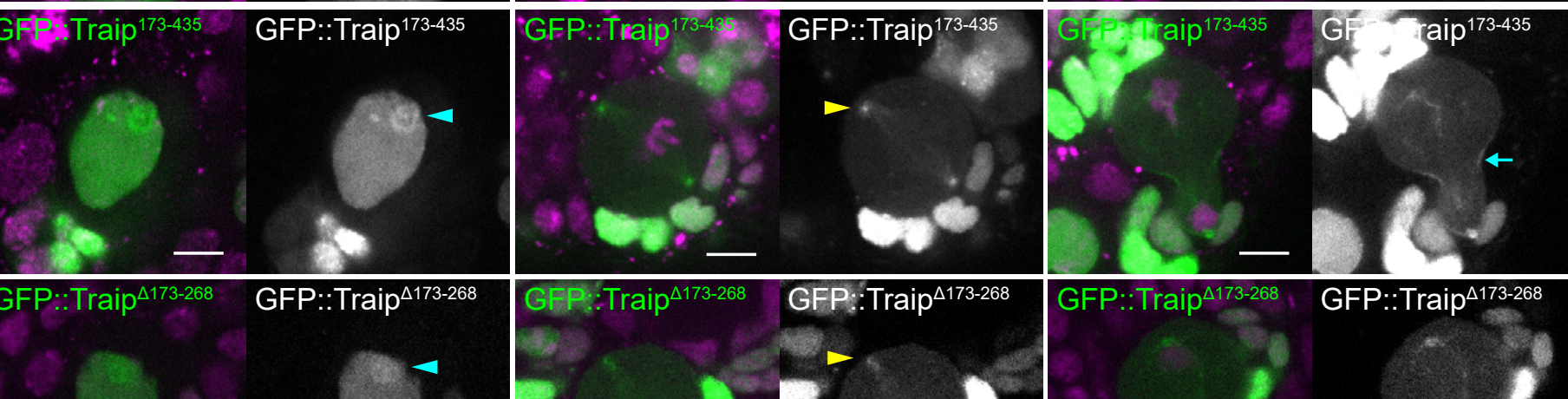

68

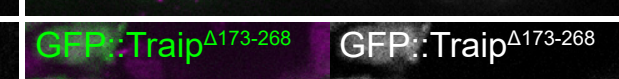

GFP::Traip ${ }^{\Delta 173-2}$

GFP::Traip ${ }^{\Delta 173-268}$

Figure S5 - Traip localization depends on distinct domains 


\section{Figure S5 - Traip localization depends on distinct domains}

(A) Schematic of major Traip protein features, transgene fragment breakpoints, and localization domains.

(B-H) Localization of GFP::Traip variant transgenes (green) expressed via wor-GAL4 with His2Av::mRFP (magenta) in CB-NBs during interphase, metaphase, and late anaphase/telophase. Yellow arrowhead denotes centrosome localization, cyan arrow denotes cytokinetic furrow localization, and cyan arrowhead denotes nucleolar localization. GFP::Traip variants include: (B) Full Length, reproduced from Figure 7D; (C) RING domain mutant I8D, A10E; (D) RING domain and first coiled coil 1-172; (E) second coiled coil and C-terminal domain 173-435; (F) a deletion of the second coiled coil $\Delta 173-268$; (G) deletion of the C-terminal domain $\Delta 269-435$; and $(H)$ the C-terminal domain alone 269-435. Scale bar $=5$ $\mu \mathrm{m}$. 


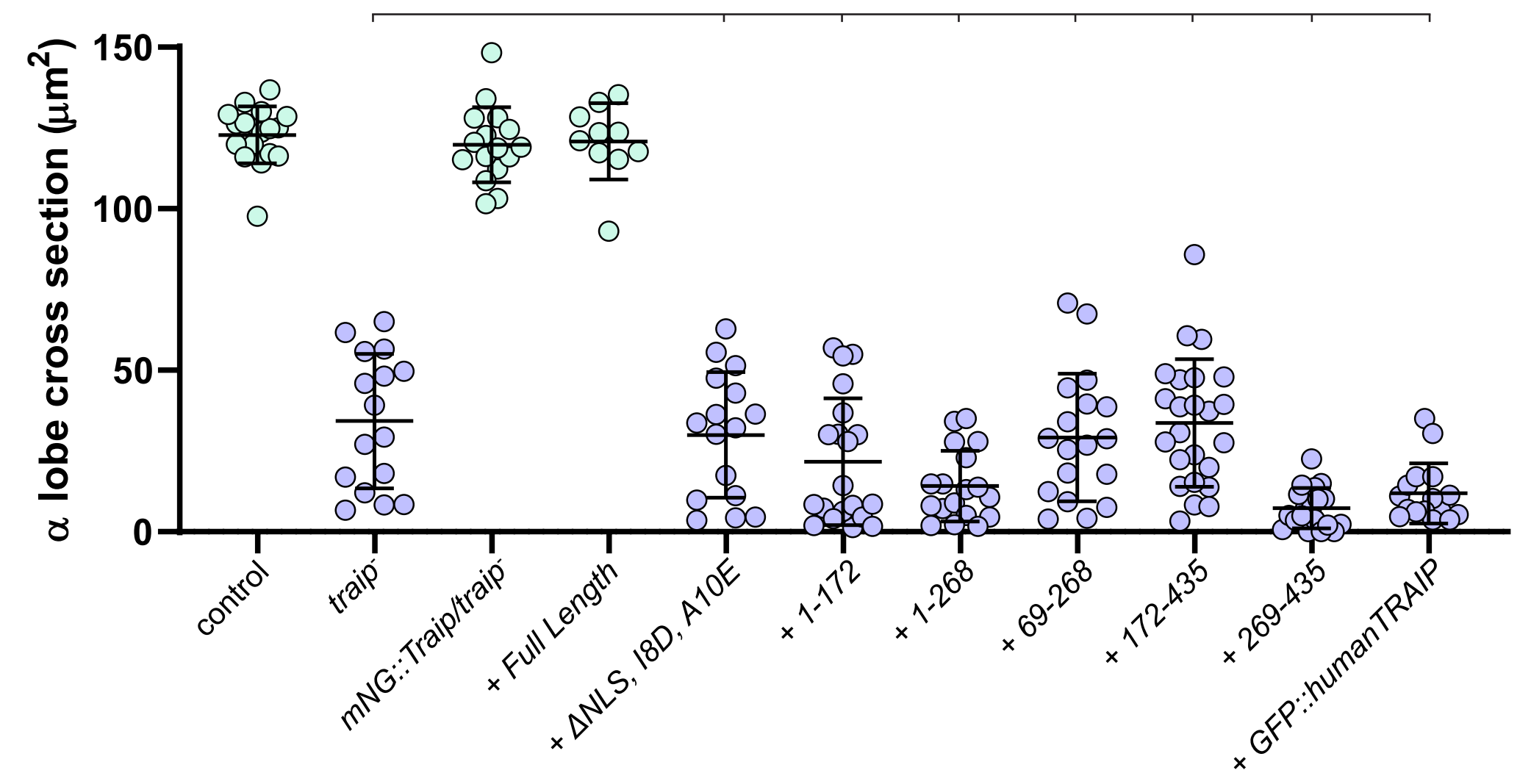

traip; OK107-GAL4 + GFP::Traip transgene variant 
bioRxiv preprint doi: https://doi.org/10.1101/2021.07.07.451466; this version posted July 7, 2021. The copyright holder for this preprint (which was not certified by peer review) is the author/funder, who has granted bioRxiv a license to display the preprint in perpetuity. It is made available under aCC-BY-NC-ND 4.0 International license.

\section{Figure S6 - Transgene rescue negative results}

(A) $\alpha$ lobe cross-section measurements of control, traip" and traip ${ }^{-}+$GFP::Traip variant transgenes expressed via OK107-GAL4. Transgenes included $m N G::$ Traip $^{\text {CRISPR }}$, Full Length, $\triangle N L S+18 D, A 10 E, 1-172$, 1-268, 69-268, 172-435, 269-435, and humanTRAIP. Ordinary one-way ANOVA with Tukey's test was used for significance. $\mathrm{ns}=$ not significant. $\mathrm{N} \geq 10 \mathrm{MBs}$. 
A control

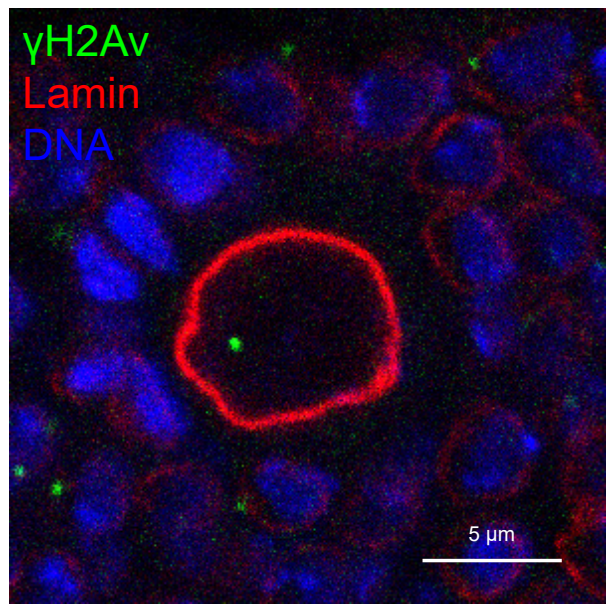
available under aCC-BY-NC-ND 4.0 International license.
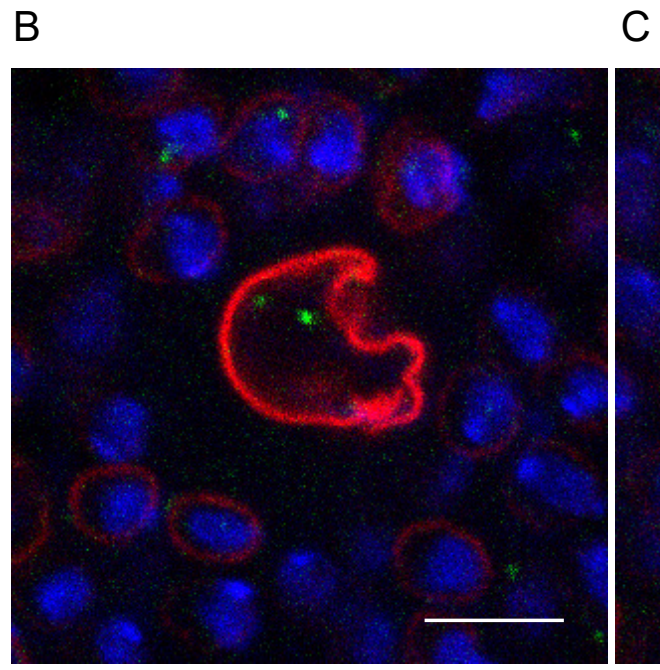

C

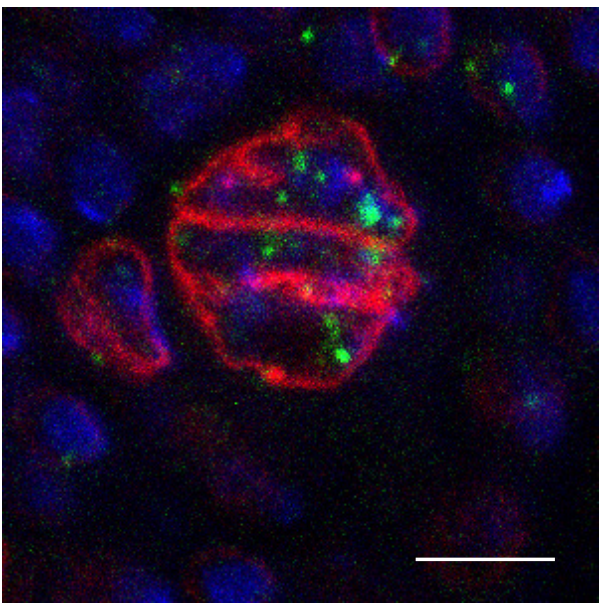

ns

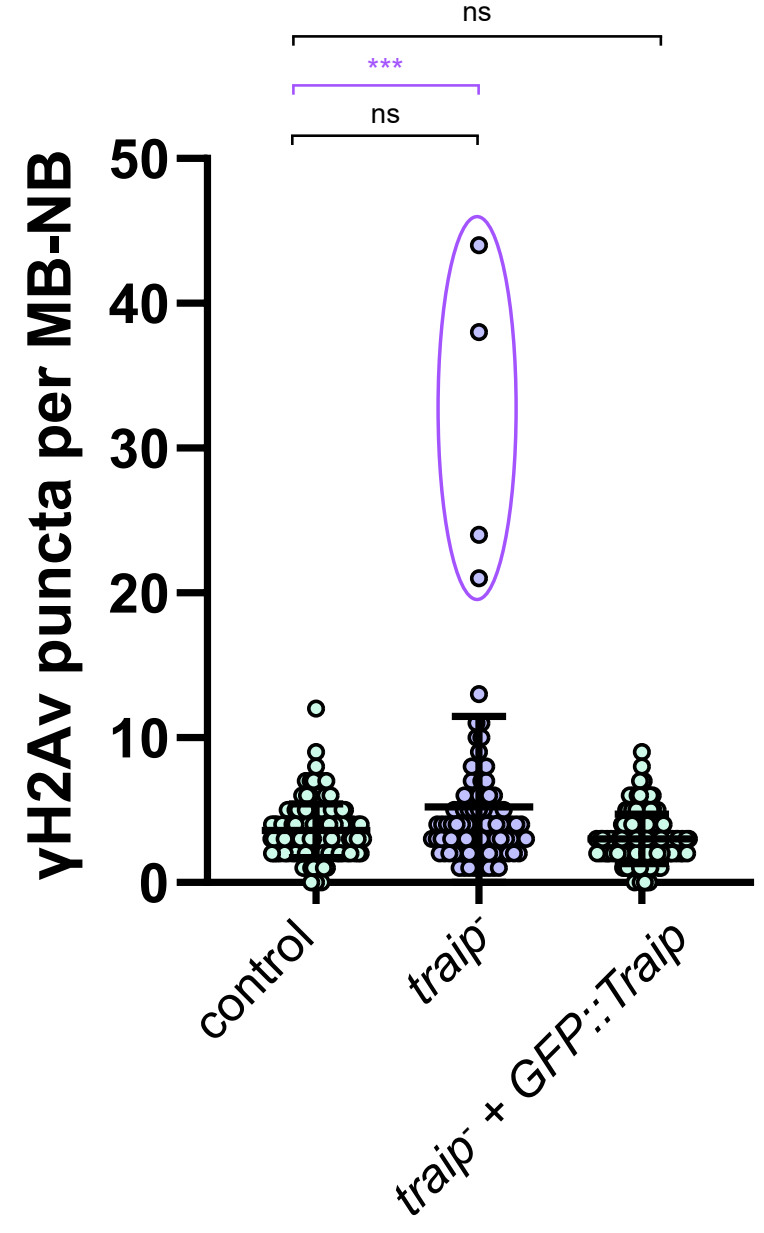

$E$
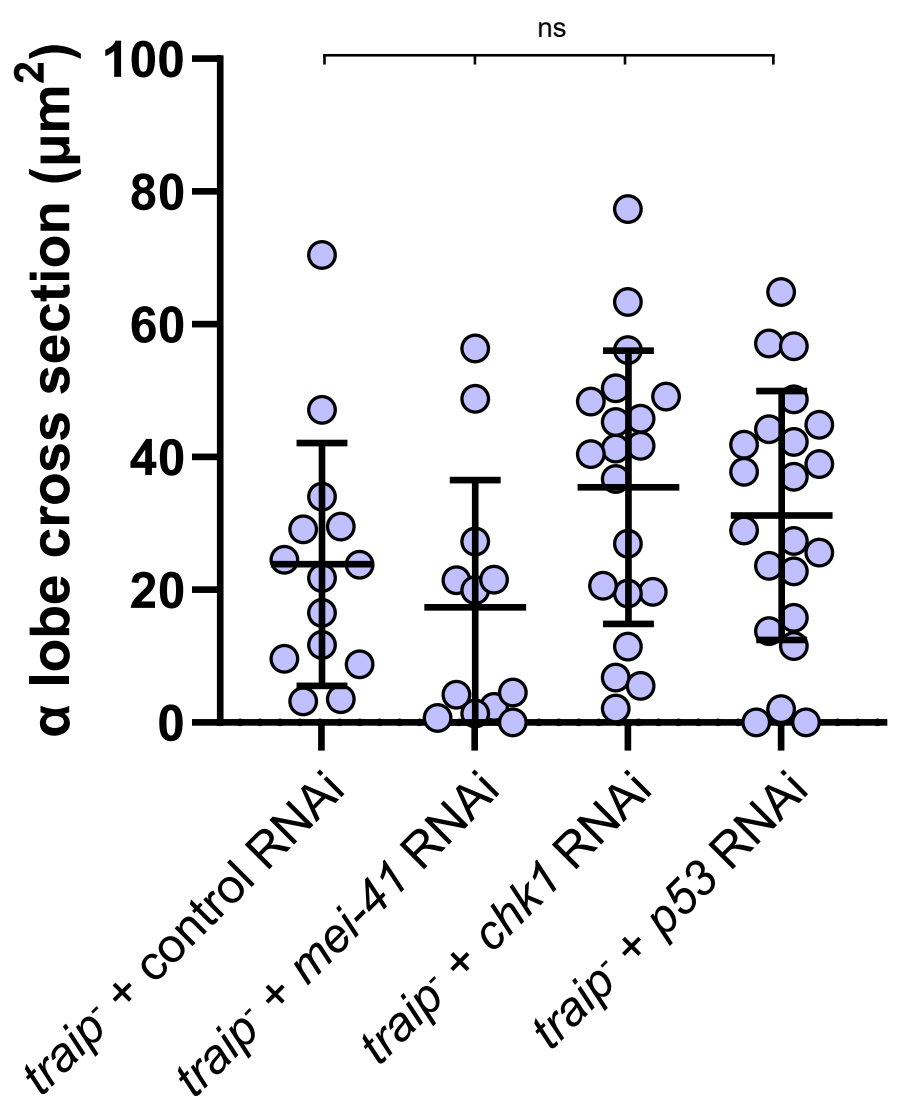


\section{Figure S7 - Traip does not function in interphase DNA damage repair}

(A-C) Control and traip 24 hours APF MB-NBs stained for $\mathrm{yH}^{-} \mathrm{Av}$ (green), Lamin (red), and DAPI (blue). Most control (A) and traip (B) MB-NBs have few $\mathrm{yH}^{-} \mathrm{Av}$ puncta. Rare, likely apoptotic traip ${ }^{-} \mathrm{MB}-\mathrm{NBs}$ have extremely elevated $\mathrm{\gamma H} 2 \mathrm{Av}$ puncta (C).

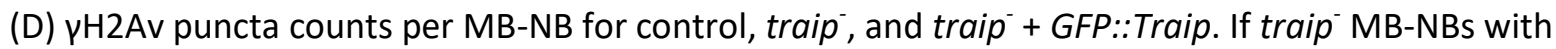
elevated $\mathrm{\gamma H} 2 \mathrm{Av}$ puncta were included (purple outline), then traip ${ }^{-} \mathrm{H} 2 \mathrm{Av}$ puncta were significantly higher than controls (purple significance bar). If these traip ${ }^{-M B-N B s}$ were excluded, then traip ${ }^{-} \mathrm{H}_{2} \mathrm{Av}$ puncta were no longer elevated (black significance bar). Ordinary one-way ANOVA with test was used for significance. $n s=$ not significant, $* * * p=0.0004, * * * p<0.0001 . N \geq 100$ MB-NBs.

(E) $\alpha$ lobe cross-section measurements of traip with control, mei-41, chk1, or $p 53$ RNAi expressed via OK107-GAL4. Two-tailed t-test was used for significance. $\mathrm{ns}=$ not significant. $\mathrm{N} \geq 12 \mathrm{MBs}$. 